\title{
Existence of Solutions with Asymptotic Expansion of Linear Partial Differential Equations in the Complex Domain
}

By

\author{
Sunao ŌUCHI*
}

\begin{abstract}
Consider the linear partial differential equation $P\left(z, \partial_{z}\right) u(z)=f(z)$ in $\mathbb{C}^{d+1}$, where $f(z)$ is not holomorphic on $K=\left\{z_{0}=0\right\}$, but it has an asymptotic expansion with respect to $z_{0}$ as $z_{0} \rightarrow 0$ in some sectorial region. We show under some conditions on $P\left(z, \partial_{z}\right)$ that there exists a solution $u(z)$ which has an asymptotic expansion of the same type as that of $f(z)$.
\end{abstract}

\section{$\S 0 . \quad$ Introduction}

Let $P\left(z, \partial_{z}\right)$ be a linear partial differential operator with holomorphic coefficients in a neighborhood $\Omega$ of $z=0$ in $\mathbb{C}^{d+1}$ and $K=\left\{z_{0}=0\right\}$. Consider the equation

$$
P\left(z, \partial_{z}\right) u(z)=f(z),
$$

where $f(z)$ is holomorphic except on $K$, but $f(z)$ has an asymptotic expansion $f(z) \sim \sum_{n} f_{n}\left(z^{\prime}\right) z_{0}^{n}$ as $z_{0} \rightarrow 0$ in some sectorial region with respect to $z_{0}$. In the present paper we study the existence of solutions. Firstly we remark that if we require nothing about the behavior of $u(z)$ near $K$, there exists a solution $u(z)$ with singularities on $K$ under some conditions on the principal symbol of $P\left(z, \partial_{z}\right)$. But the singularities of $u(z)$ may be much stronger than those of $f(z)$ (see [1], [2], [5] and [9]).

Communicated by T. Kawai. Received April 18, 2001. Revised May 17, 2002 and March 3,2003 .

2000 Mathematics Subject Classification(s): Primary 35A20; Secondary 35B40, 35C20.

Key words: complex partial differential equations, asymptotic expansion, existence of singular solutions

*Department of Mathematics, Sophia University, Chiyoda-ku, Tokyo 102-8554, Japan. e-mail: ouchi@mm.sophia.ac.jp 
It is our interest to find a solution $u(z)$ with an asymptotic expansion. The relations between the growth properties of the solution $u(z)$ and $f(z)$ near $K$ are investigated in [6], [7] and [8]. But the existence of solutions with an asymptotic expansion is not studied in those papers. It is studied in [4], where characteristic Cauchy problems are considered. Characteristic Cauchy problems have formal power series solutions. The main concern in [4] is to study the relation between genuine solutions and formal power series solutions. Our aim in the present paper is to study the existence of solutions with an asymptotic expansion in detail. We will show the following:

Suppose that $f(z) \sim \sum_{n=0}^{\infty} f_{n}\left(z^{\prime}\right) z_{0}^{n}$ in some sectorial region. Then under some conditions on $P\left(z, \partial_{z}\right)$ there exists a solution $u(z)$ with an asymptotic expansion $u(z) \sim \sum_{n=0}^{\infty} u_{n}\left(z^{\prime}\right) z_{0}^{n}$, and moreover, the asymptotic expansions of $f(z)$ and $u(z)$ are the same Gevrey type.

The plan of this paper is as follows. In Section 1 firstly we introduce function spaces $\mathcal{O}(\Omega(\theta))$ and $\operatorname{Asy}_{\{\kappa\}}(\Omega(\theta))$. $\mathcal{O}(\Omega(\theta))$ consists of holomorphic functions on the sectorial region $\Omega(\theta)$ and $\operatorname{Asy}_{\{\kappa\}}(\Omega(\theta))$ is a subspace of $\mathcal{O}(\Omega(\theta)$ ) consisting of functions with an asymptotic expansion in $z_{0}$ of Gevrey type with exponent $\kappa$. Secondly we define characteristic polygon $\Sigma$ of $P\left(z, \partial_{z}\right)$ with respect to $K$, the indices $\gamma_{i}$ and polynomials $\chi_{P, i}\left(z^{\prime}, \xi^{\prime}\right)$. The structure of the lower order terms of $P\left(z, \partial_{z}\right)$ is indispensable for the study of behavior of solutions. The characteristic polygon is defined by using the total symbol and contains information of the lower order terms. Hence it is available for our purpose. Finally we give the main results, that is, existence of functions satisfying (0.1) modulo functions with zero expansion of some Gevrey type (Theorem 1.2) and existence of an exact solution with asymptotic expansion (Theorem 1.3), and a few examples. The main result in [4] follows from Theorem 1.3. Theorem 1.3 follows easily from Theorem 1.2. Sections 2 through 6 are devoted to prove Theorem 1.2. We show Theorem 1.2 by constructing a parametrix (regularizer). In Section 2 we introduce auxiliary functions $\hat{g}_{p}(\lambda)(p \in \mathbb{Z})$ containing parameters, and $K_{p}(t)=\int_{1}^{\infty} \exp (-\lambda t) \hat{g}_{p}(\lambda) d \lambda$. We try to find a parametrix $G$ of the form

$$
(G f)(z)=\int_{\mathcal{C}} G(z, w) f(w) d w, w=\left(w_{0}, w^{\prime}\right) \in \mathbb{C} \times \mathbb{C}^{d},
$$

which is an integral operator with kernel $G(z, w)$. In Section 3 we construct formally $G(z, w)$ by a series,

$$
G(z, w)=\sum_{p \in \mathbb{Z}, q \in \mathbb{N}} k_{p, q}\left(z, w^{\prime}\right) K_{p, q}\left(w_{0}-z_{0}, w_{0}\right)
$$


where $K_{p, q}\left(w_{0}-z_{0}, w_{0}\right)=w_{0}^{q}\left(-\partial_{w_{0}}\right)^{q} K_{p}\left(w_{0}-z_{0}\right)$. We find the equations that determine the coefficients $k_{p, q}\left(z, w^{\prime}\right)$ and show that we can solve them. In Section 4 we give the estimates of $k_{p, q}\left(z, w^{\prime}\right)$ (Theorem 4.1) without proof and show the convergence of the series. We study the properties of the operator $G$, show that it is a desired parametrix and obtain Theorem 1.2. In the process we do not give the proofs of Proposition 2.3 and Theorem 4.1. We prove Proposition 2.3 in Section 5 and Theorem 4.1 in Section 6.

\section{$\S 1$. Notations and Results}

Let us give notations and definitions in order to state more precisely the problem and results. The coordinates of $\mathbb{C}^{d+1}$ are denoted by $z=\left(z_{0}, z_{1}, \ldots\right.$, $\left.z_{d}\right)=\left(z_{0}, z^{\prime}\right) \in \mathbb{C} \times \mathbb{C}^{d} .|z|=\max \left\{\left|z_{i}\right| ; 0 \leq i \leq d\right\}$ and $\left|z^{\prime}\right|=\max \left\{\left|z_{i}\right| ; 1 \leq\right.$ $i \leq d\}$. Its dual variables are $\xi=\left(\xi_{0}, \xi^{\prime}\right)=\left(\xi_{0}, \xi_{1}, \ldots, \xi_{d}\right)$. $\mathbb{N}$ is the set of all nonnegative integers $\mathbb{N}=\{0,1,2, \ldots\}$. For real number $a,[a]$ means the integral part of $a$. The partial differentiation with respect to $z_{i}$ is denoted by $\partial_{z_{i}}$, and $\partial_{z}=\left(\partial_{z_{0}}, \partial_{z_{1}}, \ldots, \partial_{z_{d}}\right)=\left(\partial_{z_{0}}, \partial_{z^{\prime}}\right)$. For a multi-index $\alpha=\left(\alpha_{0}, \alpha^{\prime}\right) \in$ $\mathbb{N} \times \mathbb{N}^{d},|\alpha|=\alpha_{0}+\left|\alpha^{\prime}\right|=\sum_{i=0}^{d} \alpha_{i}$. We use the notations $\partial_{z}^{\alpha}=\prod_{i=0}^{d} \partial_{z_{i}}^{\alpha_{i}}$ and $\partial_{z}^{\alpha^{\prime}}=\prod_{i=1}^{d} \partial_{z_{i}}^{\alpha_{i}}$. The differentiations with respect to other variables $w_{i}, \lambda, \ldots$, are denoted by $\partial_{w_{i}}, \partial_{\lambda}, \ldots$, respectively.

Let us define spaces of holomorphic functions on some regions. Let $\Omega=$ $\Omega_{0} \times \Omega^{\prime}$ be a polydisk with $\Omega_{0}=\left\{z_{0} \in \mathbb{C}^{1} ;\left|z_{0}\right|<R_{0}\right\}$ and $\Omega^{\prime}=\left\{z^{\prime} \in\right.$ $\left.\mathbb{C}^{d} ;\left|z^{\prime}\right|<R\right\}$ for some positive constants $R_{0}$ and $R$. Put $\Omega_{0}(\theta)=\left\{z_{0} \in\right.$ $\left.\Omega_{0}-\{0\} ;\left|\arg z_{0}\right|<\theta\right\}$ and $\Omega(\theta)=\Omega_{0}(\theta) \times \Omega^{\prime} . \Omega(\theta)$ is a sectorial region with respect to $z_{0}$. $\mathcal{O}(\Omega)\left(\mathcal{O}\left(\Omega^{\prime}\right), \mathcal{O}(\Omega(\theta))\right)$ is the set of all holomorphic functions on $\Omega\left(\right.$ resp. $\left.\Omega^{\prime}, \Omega(\theta)\right)$.

Definition 1.1. (i) $\operatorname{Asy}_{\{\kappa\}}(\Omega(\theta)) \quad(0<\kappa \leq+\infty)$ is the set of all $u(z) \in \mathcal{O}(\Omega(\theta))$ such that for any $\theta^{\prime}$ with $0<\theta^{\prime}<\theta$

$$
\left|u(z)-\sum_{n=0}^{N-1} u_{n}\left(z^{\prime}\right) z_{0}^{n}\right| \leq A B^{N}\left|z_{0}\right|^{N} \Gamma\left(\frac{N}{\kappa}+1\right) \quad z \in \Omega\left(\theta^{\prime}\right),
$$

where $u_{n}\left(z^{\prime}\right) \in \mathcal{O}\left(\Omega^{\prime}\right)(n \in \mathbb{N})$, holds for constants $A=A\left(\theta^{\prime}\right)$ and $B=B\left(\theta^{\prime}\right)$.

(ii) $\operatorname{Asy}_{\{0\}}(\Omega(\theta))$ is the set of all $u(z) \in \mathcal{O}(\Omega(\theta))$ such that for any $\theta^{\prime}$ with $0<\theta^{\prime}<\theta$

$$
\left|u(z)-\sum_{n=0}^{N-1} u_{n}\left(z^{\prime}\right) z_{0}^{n}\right| \leq A_{N}\left|z_{0}\right|^{N} \quad z \in \Omega\left(\theta^{\prime}\right),
$$

where $u_{n}\left(z^{\prime}\right) \in \mathcal{O}\left(\Omega^{\prime}\right)(n \in \mathbb{N})$, holds for a constant $A_{N}=A\left(N, \theta^{\prime}\right)$ depending on $N$ and $\theta^{\prime}$. 
We say that $u(z) \in \operatorname{Asy}_{\{\kappa\}}(\Omega(\theta))$ has an asymptotic expansion with Gevrey exponent (or index) $\kappa$, if $\kappa>0$. Suppose that $u(z) \in \operatorname{Asy}_{\{\kappa\}}(\Omega(\theta))$ with $\kappa>0$ and let $V \Subset \Omega$ be a polydisk centered at the origin. Then it follows from the definition that for any $0<\theta^{\prime}<\theta$ there are constants $M$ and $F$ such that

$$
\left|\partial_{z_{0}}^{n} u(z)\right| \leq M F^{n} \Gamma\left(\frac{n}{\delta}+1\right) \quad \text { for } z \in V\left(\theta^{\prime}\right), \quad \delta=\frac{\kappa}{\kappa+1} .
$$

If $u(z) \in \operatorname{Asy}_{\{+\infty\}}(\Omega(\theta))$, then $u(z)$ is holomorphic at $z=0$. If $u(z) \in$ $\operatorname{Asy}_{\{0\}}(\Omega(\theta))$, then it has merely an asymptotic expansion.

Now let $P\left(z, \partial_{z}\right)$ be an $m$-th order linear partial differential operator with holomorphic coefficients in a neighborhood of $z=0$,

$$
P\left(z, \partial_{z}\right)=\sum_{|\alpha| \leq m} a_{\alpha}(z) \partial_{z}^{\alpha}
$$

As we said, our interest is the existence of a solution $u(z)$ with an asymptotic expansion. Our problem is as follows:

Does the equation

$$
P\left(z, \partial_{z}\right) u(z)=f(z) \in \operatorname{Asy}_{\{\gamma\}}(\Omega(\theta))
$$

have a solution $u(z) \in \operatorname{Asy}_{\{\gamma\}}\left(U\left(\theta^{\prime}\right)\right)$ for some polydisk $U$ and $0<\theta^{\prime}<\theta$.

In order to answer the problem we introduce the characteristic polygon of $P\left(z, \partial_{z}\right)$. Let $j_{\alpha}$ be the valuation of $a_{\alpha}(z)$ with respect to $z_{0}$. Namely if $a_{\alpha}(z) \not \equiv$ $0, a_{\alpha}(z)=z_{0}^{j_{\alpha}} b_{\alpha}(z)$ with $b_{\alpha}\left(0, z^{\prime}\right) \not \equiv 0$. If $a_{\alpha}(z) \equiv 0$, put $j_{\alpha}=\infty$. So

$$
P\left(z, \partial_{z}\right)=\sum_{|\alpha| \leq m} z_{0}^{j_{\alpha}} b_{\alpha}(z) \partial_{z}^{\alpha} .
$$

Put

$$
e_{\alpha}=j_{\alpha}-\alpha_{0}
$$

where $e_{\alpha}=+\infty$ if $a_{\alpha}(z) \equiv 0$. We denote by $\downarrow(a, b)$ the set $\left\{(x, y) \in \mathbb{R}^{2} ; x \leq\right.$ $a, y \geq b\}$. The characteristic polygon $\Sigma$ is defined by $\Sigma:=$ the convex hull of $\bigcup_{\alpha} \downarrow\left(|\alpha|, e_{\alpha}\right)$.

The boundary of $\Sigma$ consists of a vertical half line $\Sigma(0)$, a horizontal half line $\Sigma\left(p^{*}\right)$ and $p^{*}-1$ segments $\Sigma(i)\left(1 \leq i \leq p^{*}-1\right)$ with slope $\gamma_{i}, 0=\gamma_{p^{*}}<$ $\gamma_{p^{*}-1}<\cdots<\gamma_{1}<\gamma_{0}=+\infty$. Let $\left\{\left(k_{i}, e(i)\right) \in \boldsymbol{R}^{2} ; 0 \leq i \leq p^{*}-1\right\}$ be the vertices of $\Sigma$, where $0 \leq k_{p^{*}-1}<\cdots<k_{i}<k_{i-1}<\cdots<k_{0}=m$. So the endpoints of $\Sigma(i)\left(1 \leq i \leq p^{*}-1\right)$ are $\left(k_{i-1}, e(i-1)\right)$ and $\left(k_{i}, e(i)\right)$. We call $\gamma_{i}$ the $i$-th characteristic index of $P(z, \partial)$ with respect to $K=\left\{z_{0}=0\right\}$. For each 


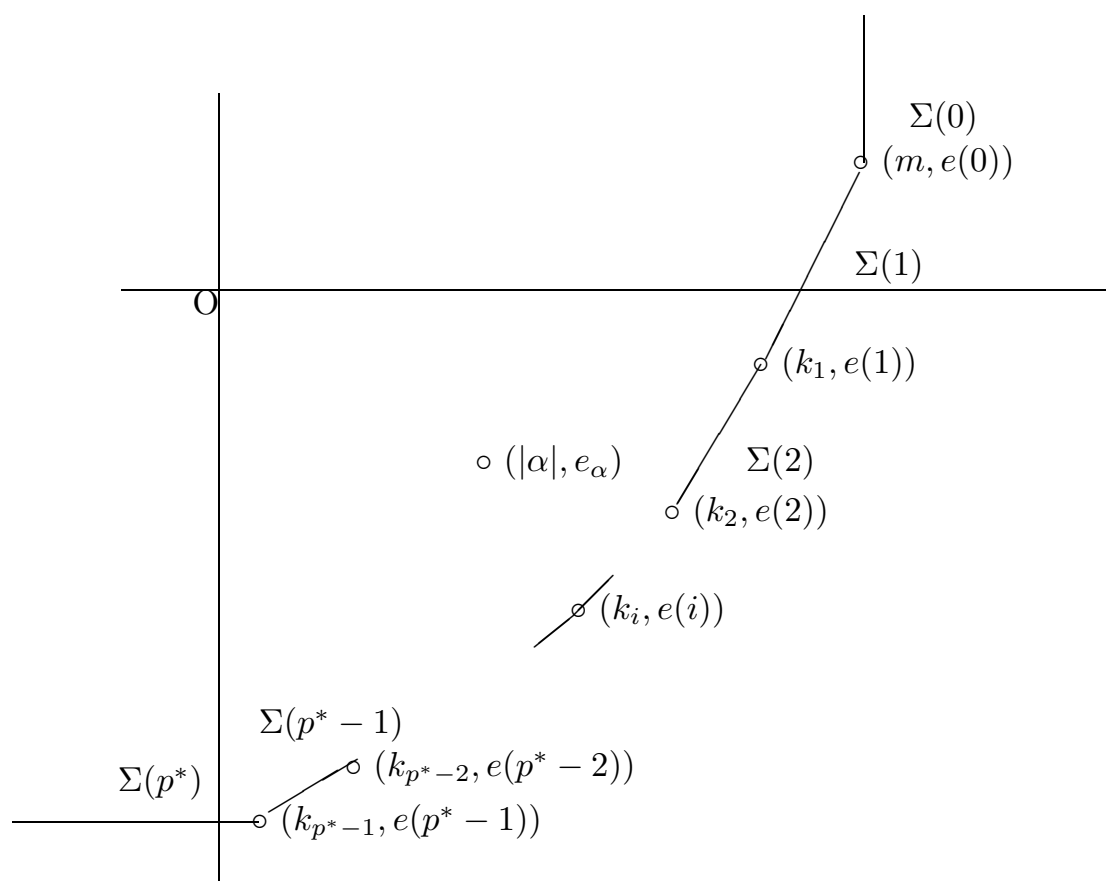

Figure 1. Characteristic polygon

$i\left(0 \leq i \leq p^{*}-1\right)$ define subsets $\Delta(i)$ of multi-indices and quantities $l_{i} \in \mathbb{N}$ as follows:

$$
\left\{\begin{aligned}
\Delta(i) & :=\left\{\alpha \in \mathbb{N}^{d+1} ;|\alpha|=k_{i}, e_{\alpha}=e(i)\right\}, \\
l_{i} & :=\max \left\{\left|\alpha^{\prime}\right|: \alpha \in \Delta(i)\right\} .
\end{aligned}\right.
$$

Define a subset $\Delta_{0}(i)$ of $\Delta(i)$ and a polynomial $\chi_{P, i}\left(z^{\prime}, \xi^{\prime}\right)$ in $\xi^{\prime}$ by

$$
\left\{\begin{aligned}
\Delta_{0}(i) & =\left\{\alpha \in \Delta(i) ;\left|\alpha^{\prime}\right|=l_{i}\right\} \\
\chi_{P, i}\left(z^{\prime}, \xi^{\prime}\right) & =\sum_{\alpha \in \Delta_{0}(i)} b_{\alpha}\left(0, z^{\prime}\right) \xi^{\alpha^{\prime}}
\end{aligned}\right.
$$

$\chi_{P, i}\left(z^{\prime}, \xi^{\prime}\right)$ is homogeneous in $\xi^{\prime}$ with degree $l_{i}$.

We give conditions on $P\left(z, \partial_{z}\right)$ denoted by $\left(\mathrm{C}_{i}\right) \quad\left(0 \leq i \leq p^{*}-1\right)$. For each fixed $i$

$\left(\mathrm{C}_{i}\right) j_{\alpha}=0$ for all $\alpha \in \Delta_{0}(i)$ and $\chi_{P, i}\left(0, \xi^{\prime}\right) \not \equiv 0$. 
If $P\left(z, \partial_{z}\right)$ satisfies $\left(\mathrm{C}_{i}\right)$, then $e(i)=-k_{i}+l_{i}$ and $b_{\alpha}\left(0,0^{\prime}\right) \neq 0$ for some $\alpha=\left(k_{i}-l_{i}, \alpha^{\prime}\right) \in \Delta_{0}(i)$.

Now let us return to the existence of solutions of

$$
P\left(z, \partial_{z}\right) u(z)=f(z) \in \operatorname{Asy}_{\{\gamma\}}(\Omega(\theta)) .
$$

Firstly we have

Theorem 1.2. $\quad$ Suppose that $P\left(z, \partial_{z}\right)$ satisfies $\left(\mathrm{C}_{i}\right)$ for some $i \in\{0$, $\left.1, \ldots, p^{*}-1\right\}$ and $f(z) \in \operatorname{Asy}_{\{\gamma\}}(\Omega(\theta))$ with $\gamma_{i+1} \leq \gamma<\gamma_{i}$. Let $\theta^{\prime}$ be a constant such that $0<\theta^{\prime}<\min \left\{\theta, \pi / 2 \gamma_{i}\right\}$ if $i \neq 0$, and $0<\theta^{\prime}<\theta$ if $i=0$. Then there exists $g(z) \in \operatorname{Asy}_{\{\gamma\}}\left(U\left(\theta^{\prime}\right)\right)$ for some polydisk $U$ centered at the origin such that

$$
(R f)(z):=\left(P\left(z, \partial_{z}\right) g(z)-f(z)\right) \sim 0
$$

in $\operatorname{Asy}_{\left\{\gamma_{i}\right\}}\left(U\left(\theta^{\prime}\right)\right)$.

This theorem implies if $i=0$, then $(R f)(z) \sim 0$ in $\operatorname{Asy}_{\{+\infty\}}\left(U\left(\theta^{\prime}\right)\right)$. This means that $(R f)(z)$ is holomorphic at $z=0$ and has zero expansion, hence $(R f)(z) \equiv 0$ and $P\left(z, \partial_{z}\right) g(z)=f(z)$.

By using Theorem 1.2 repeatedly, we have the following existence of a solution $u(z)$ whose asymptotic expansion is the same Gevrey type as that of $f(z)$.

Theorem 1.3. Suppose that $P\left(z, \partial_{z}\right)$ satisfies $\left(\mathrm{C}_{i}\right)$ for $i=0,1, \ldots, s$, and let $f(z) \in \operatorname{Asy}_{\{\gamma\}}(\Omega(\theta))$ with $\gamma \geq \gamma_{s+1}$. Then for any $0<\theta^{\prime}<\min \{\theta$, $\left.\pi / 2 \gamma_{1}\right\}$ there exists $u(z) \in \operatorname{Asy}_{\{\gamma\}}\left(U\left(\theta^{\prime}\right)\right)$ satisfying $P\left(z, \partial_{z}\right) u(z)=f(z)$ for some polydisk $U$ centered at the origin.

As for the dependence of the polydisk $U$ on $\theta^{\prime}$ and $f(z)$, we refer to Theorems 4.7 and 4.8 .

We give examples. Let $P\left(z, \partial_{z}\right)$ be a noncharacteristic operator with respect to $z_{0}=0$. Then $+\infty=\gamma_{0}>\gamma_{1}=0$. Consider $P\left(z, \partial_{z}\right) u(z)=f(z) \in$ $\operatorname{Asy}_{\{\gamma\}}(\Omega(\theta))$ with $\gamma \geq \gamma_{1}(=0)$. Let $0<\theta^{\prime}<\theta$. Then we have a solution $u(z) \in \operatorname{Asy}_{\{\gamma\}}\left(U\left(\theta^{\prime}\right)\right)$ for some polydisk $U$ by Theorem 1.3.

The following examples are characteristic with respect to $z_{0}=0$. A simple example is

$$
P(z, \partial)=\partial_{z_{1}}^{2}-\partial_{z_{0}}, \quad z=\left(z_{0}, z_{1}\right) \in \mathbb{C}^{2}
$$


We have $\gamma_{0}=+\infty, \gamma_{1}=1, \gamma_{2}=0, \chi_{P, 0}\left(z^{\prime}, \xi_{1}\right)=\xi_{1}^{2}$ and $\chi_{P, 1}\left(z^{\prime}, \xi_{1}\right)=-1$. Consider $P\left(z, \partial_{z}\right) u(z)=f(z) \in \operatorname{Asy}_{\{\gamma\}}(\Omega(\theta))$ with $\gamma \geq \gamma_{2}(=0)$. Let $0<\theta^{\prime}<$ $\min \{\theta, \pi / 2\}$. Then we have a solution $u(z) \in \operatorname{Asy}_{\{\gamma\}}\left(U\left(\theta^{\prime}\right)\right)$ by Theorem 1.3.

We give further two examples. One is

$$
P(z, \partial)=\partial_{z_{1}}^{5}+\partial_{z_{1}}^{3} \partial_{z_{0}}+\partial_{z_{0}}^{2}, \quad z=\left(z_{0}, z_{1}\right) \in \mathbb{C}^{2} .
$$

We have

$$
\left\{\begin{array}{l}
\gamma_{0}=+\infty, \quad \gamma_{1}=1, \quad \gamma_{2}=1 / 2, \quad \gamma_{3}=0, \\
\chi_{P, 0}\left(z^{\prime}, \xi_{1}\right)=\xi_{1}^{5}, \quad \chi_{P, 1}\left(z^{\prime}, \xi_{1}\right)=\xi_{1}^{3}, \quad \chi_{P, 2}\left(z^{\prime}, \xi_{1}\right)=1 .
\end{array}\right.
$$

Obviously $P\left(z, \partial_{z}\right)$ satisfies $\left(\mathrm{C}_{i}\right)$ for $i=0,1,2$. Consider $P\left(z, \partial_{z}\right) u(z)=f(z) \in$ $\operatorname{Asy}_{\{\gamma\}}(\Omega(\theta))$ with $\gamma \geq \gamma_{3}(=0)$. Let $0<\theta^{\prime}<\min \{\theta, \pi / 2\}$. Then we have a solution $u(z) \in \operatorname{Asy}_{\{\gamma\}}\left(U\left(\theta^{\prime}\right)\right)$ by Theorem 1.3. Another is

$$
P(z, \partial)=\partial_{z_{1}}^{8}+\partial_{z_{1}}^{5} \partial_{z_{0}}^{2}+z_{0} \partial_{z_{1}}^{2} \partial_{z_{0}}^{4}+\partial_{z_{0}}^{4}, \quad z=\left(z_{0}, z_{1}\right) \in \mathbb{C}^{2} .
$$

We have

$$
\left\{\begin{array}{l}
\gamma_{0}=+\infty, \quad \gamma_{1}=2, \quad \gamma_{2}=1, \quad \gamma_{3}=1 / 2, \quad \gamma_{4}=0 \\
\chi_{P, 0}\left(z^{\prime}, \xi_{1}\right)=\xi_{1}^{8}, \chi_{P, 1}\left(z^{\prime}, \xi_{1}\right)=\xi_{1}^{5}, \chi_{P, 2}\left(z^{\prime}, \xi_{1}\right)=\xi_{1}^{2}, \chi_{P, 3}\left(z^{\prime}, \xi_{1}\right)=1 .
\end{array}\right.
$$

Consider $P\left(z, \partial_{z}\right) u(z)=f(z) \in \operatorname{Asy}_{\{\gamma\}}(\Omega(\theta))$. $P\left(z, \partial_{z}\right)$ satisfies $\left(\mathrm{C}_{i}\right)$ for $i=$ $0,1,3$ but $\left(\mathrm{C}_{2}\right)$ does not hold. Hence by Theorem 1.3 if $\gamma \geq \gamma_{2}$, there is a solution $u(z) \in \operatorname{Asy}_{\{\gamma\}}\left(U\left(\theta^{\prime}\right)\right)$ for some polydisk $U$, where $0<\theta^{\prime}<\min \{\theta, \pi / 4\}$.

\section{§2. Auxiliary Functions}

We show Theorem 1.2 by finding parametrices of $P\left(z, \partial_{z}\right)$. In order to construct them we need some auxiliary functions. Let us introduce them and give their elementary properties as lemmas and propositions in this section.

Let $0<\delta \leq 1$ and

$$
\hat{g}_{p}(\lambda)=\left\{\begin{array}{l}
\frac{\lambda^{\delta}}{\Gamma\left(\frac{p}{\delta}+1\right)} \int_{0}^{d} \exp \left(-\lambda^{\delta} \zeta\right) \zeta^{\frac{p}{\delta}} d \zeta \quad \text { for } \quad p>0 \\
\lambda^{-p} \quad \text { for } \quad p \leq 0 .
\end{array}\right.
$$

Here $d>0$ is a parameter, which will be chosen so small and fixed later. We remark that the dimension of $z^{\prime}$ is also denoted by $d, z^{\prime}=\left(z_{1}, z_{2}, \ldots, z_{d}\right) \in \mathbb{C}^{d}$, but there will be no confusion. $\hat{g}_{p}(\lambda)$ depends on $\delta$ and $d$ if $p>0$, but $\hat{g}_{p}(\lambda)$ 
does not if $p \leq 0$. In the following we assume $p \in \mathbb{Z}$ and denote $\Gamma(x+1)$ by $x$ !. Define

$$
K_{p}(\delta ; t)=\frac{1}{2 \pi i} \int_{1}^{\infty} \exp (-\lambda t) \hat{g}_{p}(\lambda) d \lambda .
$$

It is obvious that $K_{p}(\delta ; t)$ is multi-valued holomorphic on $\{t \neq 0\}$ for $0<\delta<1$. It holds that if $p>0$,

$$
\begin{aligned}
K_{p}(\delta ; t) & =\frac{1}{2 \pi i} \int_{e^{i \phi}}^{\infty e^{i \phi}} \exp (-\lambda t) \hat{g}_{p}(\lambda) d \lambda+\frac{1}{2 \pi i} \int_{1}^{e^{i \phi}} \exp (-\lambda t) \hat{g}_{p}(\lambda) d \lambda \\
& =K_{p}^{*}(\delta ; t)+K_{p}^{* *}(\delta ; t),
\end{aligned}
$$

where $K_{p}^{* *}(\delta ; t)$ is an entire function.

Lemma 2.1. (1) Let $0<\delta<1$ and $\phi_{0}$ be an arbitrary constant with $0<\phi_{0}<\frac{\pi}{2 \delta}$. Suppose $|\arg t|<\phi_{0}+\frac{\pi}{2}$ and $0<|t| \leq T$. Then there exists a constant $A=A\left(\phi_{0}, T\right)$ such that

$$
\left|K_{p}(\delta ; t)\right| \leq \begin{cases}A d^{\frac{p-2}{\delta}} /\left(\frac{p}{\delta}\right) ! & \text { for } p \geq 2 \\ A(1+|\log t|) & \text { for } p=1\end{cases}
$$

(2) Let $\delta=1$ and $p \geq 1$. Then

$$
K_{p}(\delta ; t)=\frac{(-1)^{p} t^{p-1} \log t}{2 \pi i(p-1) !}+\text { a holomorphic function on }\{|t|<d\} .
$$

(3) Let $p \leq 0$. Then

$$
K_{p}(\delta ; t)=\frac{|p| !}{2 \pi i t|p|+1}+\text { an entire function in } t .
$$

Proof. (1) The constant $A$ means various constants depending on $\phi_{0}$ and $T$ in the following inequalities. Let $\epsilon$ be a small positive constant with $\epsilon<\frac{\pi}{2 \delta}-$ $\phi_{0}$. Set $\theta=\arg t$. Since $|\theta|<\phi_{0}+\frac{\pi}{2}$, there exists $\phi$ with $|\phi|<\phi_{0}+\epsilon<\pi / 2 \delta$ such that $|\phi+\theta|<\pi / 2-\epsilon$. Hence $\cos (\phi+\theta)>\sin \epsilon>0$ and $\cos \delta \phi>\cos \delta\left(\phi_{0}+\epsilon\right)>0$. Take $\arg \lambda=\phi$. Let $p \geq 2$. Then it holds that

$$
\begin{aligned}
\left|K_{p}^{*}(\delta ; t)\right| & \leq \frac{1}{2 \pi\left(\frac{p}{\delta}\right) !} \int_{1}^{\infty} r^{\delta} \exp (-r|t| \cos (\phi+\theta)) d r \int_{0}^{d} \exp \left(-r^{\delta} \zeta \cos \delta \phi\right) \zeta^{\frac{2}{\delta}+\frac{p-2}{\delta}} d \zeta \\
& \leq \frac{d^{\frac{p-2}{\delta}}}{2 \pi\left(\frac{p}{\delta}\right) !} \int_{1}^{\infty} r^{\delta} \exp (-r|t| \cos (\phi+\theta)) d r \int_{0}^{d} \exp \left(-r^{\delta} \zeta \cos \delta \phi\right) \zeta^{\frac{2}{\delta}} d \zeta .
\end{aligned}
$$


$\mathrm{By}\left|r^{\delta} \int_{0}^{d} \exp \left(-r^{\delta} \zeta \cos \delta \phi\right) \zeta^{\frac{2}{\delta}} d \zeta\right| \leq C r^{-2}(\cos \delta \phi)^{-\frac{2}{\delta}-1}$, we have

$$
\left|K_{p}^{*}(\delta ; t)\right| \leq \frac{A d^{\frac{p-2}{\delta}}}{(\cos \delta \phi)^{\frac{2}{\delta}+1}\left(\frac{p}{\delta}\right) !} \int_{1}^{\infty} \frac{\exp (-r|t| \cos (\phi+\theta))}{r^{2}} d r \leq \frac{A d^{\frac{p-2}{\delta}}}{\left(\frac{p}{\delta}\right) !} .
$$

Let $p=1$. Then we have

$$
\begin{aligned}
\left|K_{1}^{*}(\delta ; t)\right| & \leq \frac{1}{2 \pi\left(\frac{1}{\delta}\right) !} \int_{1}^{\infty} r^{\delta} \exp (-r|t| \cos (\phi+\theta)) d r \int_{0}^{d} \exp \left(-r^{\delta} \zeta \cos \delta \phi\right) \zeta^{\frac{1}{\delta}} d \zeta \\
& \leq A \int_{1}^{\infty} \frac{\exp (-r|t| \sin \epsilon)}{r} d r=A \int_{|t| \sin \epsilon}^{\infty} \frac{\exp (-r)}{r} d r \\
& \leq A(1+|\log t|) .
\end{aligned}
$$

It remains to estimate $K_{p}^{* *}(\delta ; t)$. However it is easy and we have (2.4).

(2) Suppose $\delta=1$. Then $\hat{g}_{1}(\lambda)=\left(1-(1+\lambda d) e^{-\lambda d}\right) / \lambda$. Hence

$$
\begin{aligned}
K_{1}(1 ; t) & =\frac{1}{2 \pi i} \int_{1}^{\infty} \frac{\exp (-\lambda t)}{\lambda} d \lambda-\frac{1}{2 \pi i} \int_{1}^{\infty} \frac{(1+\lambda d) \exp (-\lambda(t+d))}{\lambda} d \lambda \\
& \equiv \frac{1}{2 \pi i} \int_{1}^{\infty} \frac{\exp (-\lambda t)}{\lambda} d \lambda, \\
\frac{d}{d t} K_{1}(1 ; t) & \equiv \frac{-1}{2 \pi i} \int_{1}^{\infty} \exp (-\lambda t) d \lambda \equiv \frac{-1}{2 \pi i t},
\end{aligned}
$$

where $\equiv$ means modulo holomorphic functions on $\{|t|<d\}$. We also have

$$
\begin{aligned}
\frac{d}{d t} K_{p+1}(1 ; t) & =\frac{-1}{2 \pi i} \int_{1}^{\infty} \lambda \exp (-\lambda t) \hat{g}_{p+1}(\lambda) d \lambda \\
& =\frac{-1}{2 \pi i} \int_{1}^{\infty} \exp (-\lambda t) \hat{g}_{p}(\lambda) d \lambda+\frac{d^{p+1}}{2 \pi i(p+1) !} \int_{1}^{\infty} \lambda \exp (-\lambda(t+d)) d \lambda \\
& \equiv-K_{p}(\delta ; t) .
\end{aligned}
$$

By integrating $K_{p}(\delta ; t)$ successively, we have $(2.5)$.

(3) Let $p \leq 0$. Then we have

$$
K_{p}(\delta ; t)=\frac{1}{2 \pi i} \int_{1}^{\infty} \exp (-\lambda t) \lambda^{|p|} d \lambda=\frac{|p| !}{2 \pi i t|p|+1}-\frac{1}{2 \pi i} \int_{0}^{1} \exp (-\lambda t) \lambda^{|p|} d \lambda,
$$

where the last term defined by the integral on $[0,1]$ is an entire function in $t$.

The following Lemma 2.2 and Proposition 2.3 are used to construct parametrices in the following sections. In particular, the relation (2.7) appears in the calculations in Section 3. 
Lemma 2.2. Let $h \in \mathbb{Z}$ and $s \in \mathbb{N}$. Then it holds that

$$
\begin{aligned}
& \frac{1}{2 \pi i} \int_{1}^{\infty} \exp (-\lambda t) \lambda^{h}\left(-\lambda \partial_{\lambda}\right)^{s} \hat{g}_{p}(\lambda) d \lambda=p^{s} K_{p-h}(\delta ; t)+R_{p, h, s}(t) \\
& R_{p, h, s}(t)=\frac{1}{2 \pi i} \int_{1}^{\infty} \exp (-\lambda t) \hat{r}_{p, h, s}(\lambda) d \lambda
\end{aligned}
$$

where $\hat{r}_{p, h, s}(\lambda)=p^{s} \hat{r}_{p, h}^{0}(\lambda)+\lambda^{h} \hat{r}_{p, s}^{1}(\lambda)$ and

$$
\left\{\begin{array}{l}
\hat{r}_{p, h}^{0}(\lambda):=\lambda^{h} \hat{g}_{p}(\lambda)-\hat{g}_{p-h}(\lambda), \quad \hat{r}_{p, h}^{0}(\lambda)=0 \quad \text { for } p \leq 0 \text { and } h \geq 0 \\
\hat{r}_{p, s}^{1}(\lambda)=\frac{-\delta d^{\frac{p}{\delta}+1}}{\left(\frac{p}{\delta}\right) !}\left(\sum_{k=0}^{s-1} p^{s-1-k}\left(-\lambda \frac{\partial}{\partial \lambda}\right)^{k}\left(\lambda^{\delta} e^{-d \lambda^{\delta}}\right)\right) \quad \text { for } p>0 \\
\hat{r}_{p, s}^{1}(\lambda)=0 \quad \text { for } \quad p \leq 0
\end{array}\right.
$$

Proof. Let $p>0$. Then we have by integrations by parts

$$
\begin{aligned}
-\lambda \frac{\partial}{\partial \lambda} \hat{g}_{p}(\lambda) & =\frac{-\delta \lambda^{\delta}}{\left(\frac{p}{\delta}\right) !} \int_{0}^{d} \exp \left(-\lambda^{\delta} \zeta\right) \zeta^{\frac{p}{\delta}} d \zeta+\frac{\delta \lambda^{\delta}}{\left(\frac{p}{\delta}\right) !} \int_{0}^{d} \lambda^{\delta} \exp \left(-\lambda^{\delta} \zeta\right) \zeta^{\frac{p}{\delta}+1} d \zeta \\
& =p \hat{g}_{p}(\lambda)-\frac{\delta d^{\frac{p}{\delta}+1} \lambda^{\delta} e^{-d \lambda^{\delta}}}{\left(\frac{p}{\delta}\right) !}
\end{aligned}
$$

By repeating the above calculation, we have $\left(-\lambda \frac{\partial}{\partial \lambda}\right)^{s} \hat{g}_{p}(\lambda)=p^{s} \hat{g}_{p}(\lambda)+\hat{r}_{p, s}^{1}(\lambda)$, where $\hat{r}_{p, s}^{1}(\lambda)=\frac{\delta d \frac{p}{\delta}+1}{\left(\frac{p}{\delta}\right) !}\left(\sum_{k=0}^{s-1} p^{s-1-k}\left(-\lambda \frac{\partial}{\partial \lambda}\right)^{k}\left(\lambda^{\delta} e^{-d \lambda^{\delta}}\right)\right)$. If $p \leq 0$, then $\left(-\lambda \frac{\partial}{\partial \lambda}\right)^{s} \hat{g}_{p}(\lambda)=p^{s} \hat{g}_{p}(\lambda)$ and $\hat{r}_{p, s}^{1}(\lambda) \equiv 0$. Set $\hat{r}_{p, h}^{0}(\lambda):=\lambda^{h} \hat{g}_{p}(\lambda)-\hat{g}_{p-h}(\lambda)$. Note that $\hat{r}_{p, h}^{0}(\lambda)=0$ for $p \leq 0$ and $h \geq 0$. Hence $\lambda^{h}\left(-\lambda \frac{\partial}{\partial \lambda}\right)^{s} \hat{g}_{p}(\lambda)=$ $p^{s} \lambda^{h} g_{p}(\lambda)+\lambda^{h} \hat{r}_{p, s}^{1}(\lambda)=p^{s} \hat{g}_{p-h}(\lambda)+p^{s} \hat{r}_{p, h}^{0}(\lambda)+\lambda^{h} \hat{r}_{p, s}^{1}(\lambda)=p^{s} \hat{g}_{p-h}(\lambda)+$ $\hat{r}_{p, h, s}(\lambda)$ and

$$
\frac{1}{2 \pi i} \int_{1}^{\infty} \exp (-\lambda t) \lambda^{h}\left(-\lambda \frac{\partial}{\partial \lambda}\right)^{s} \hat{g}_{p}(\lambda) d \lambda=p^{s} K_{p-h}(t)+R_{p, h, s}(t) .
$$

We give estimates of $\hat{r}_{p, h, s}(\lambda)$ to study $R_{p, h, s}(t)$ defined by $(2.7)$.

Proposition 2.3. Let $\phi_{0}$ be an arbitrary constant with $0<\phi_{0}<\frac{\pi}{2 \delta}$ and $s_{0} \in \mathbb{N}$. Suppose $\lambda \in\left\{|\lambda| \geq 1 ;|\arg \lambda|<\phi_{0}\right\}$ and $0 \leq s \leq s_{0}$. Set $h_{-}=\min \{h, 0\}$ and $h_{+}=\max \{h, 0\}$. Then the following estimates hold. 
(1) If $p \leq h_{-}$, then $\hat{r}_{p, h, s}(\lambda)=0$.

(2) If $h_{-}<p<h_{+}$, then there are constants $A=A\left(\phi_{0}\right)$ and $B=B\left(\phi_{0}\right)$ such that

$$
\left|\hat{r}_{p, h, s}(\lambda)\right| \leq A B^{|h|}(1+|p|)^{s_{0}} d^{\frac{p-h_{+}}{\delta}}\left(\frac{h_{+}-p}{\delta}\right) ! e^{-\frac{d\left(\cos \delta \phi_{0}\right)}{2}|\lambda|^{\delta}} .
$$

(3) If $p \geq h_{+}$, then there are constants $A=A\left(\phi_{0}\right)$ and $B=B\left(\phi_{0}\right)$ such that

$$
\left|\hat{r}_{p, h, s}(\lambda)\right| \leq \frac{A B^{|h|}(1+|p|)^{s_{0}} d^{\frac{p-h_{+}}{\delta}} e^{-\frac{d\left(\cos \delta \phi_{0}\right)}{2}|\lambda|^{\delta}}}{\left(\frac{p-h_{+}}{\delta}\right) !} .
$$

Proposition 2.3 asserts that $\hat{r}_{p, h, s}(\lambda)$ decays exponentially with order $\delta$ as $\lambda \rightarrow \infty$ in some sectorial region. This decay estimate is important. The proof of Proposition 2.3 is given in Section 5. In this paper there appear integral operators

$$
\left(R_{p, h, s} f\right)\left(z_{0}\right)=\int_{\mathcal{C}_{0}} R_{p, h, s}\left(w_{0}-z_{0}\right) f\left(w_{0}\right) d w_{0},
$$

where the path $\mathcal{C}_{0}$ is defined later in this section. $(R f)(z)$ in Theorem 1.2 is an infinite linear combination of integral operators of the form (2.11). We study the behavior and the estimates of $\left(R_{p, h, s} f\right)\left(z_{0}\right)$. Firstly we give

Lemma 2.4. Let $\hat{r}(\lambda)$ be a holomorphic function on $\left\{\lambda ;|\arg \lambda|<\phi_{0}\right.$, $|\lambda| \geq 1\}$ with $\phi_{0}>\pi / 2$. Suppose that there are positive constants $A, c_{0}$ and $0<\delta \leq 1$ such that $|\hat{r}(\lambda)| \leq A \exp \left(-c_{0}|\lambda|^{\delta}\right)$. Define

$$
R(t)=\frac{1}{2 \pi i} \int_{1}^{\infty e^{i \phi}} \exp (-\lambda t) \hat{r}(\lambda) d \lambda .
$$

(1) If $0<\delta<1$, then $R(t) \in \operatorname{Asy}_{\{\gamma\}}\left(\left\{t \neq 0 ;|\arg t|<\phi_{0}+\frac{\pi}{2}\right\}\right)$ with $\gamma=$ $\delta /(1-\delta)$ and there are positive constants $C$ and $c$ such that

$$
\left|R(t)-R\left(t e^{2 \pi i}\right)\right| \leq C \exp \left(-c|t|^{-\gamma}\right) \text { for }|\arg t+\pi|<\phi_{0}-\frac{\pi}{2} .
$$

(2) If $\delta=1$, then $R(t) \in \mathcal{O}\left(\left\{t ;|t|<c_{0}\right\}\right)$.

Proof. Assume $0<\delta<1$. If $|\arg t+\phi|<\pi / 2$, the integral (2.12) is absolutely convergent. Hence $R(t)$ is holomorphic on $\left\{t \neq 0 ;|\arg t|<\phi_{0}+\frac{\pi}{2}\right\}$. We have

$$
\left(\frac{d}{d t}\right)^{n} R(t)=\frac{1}{2 \pi i} \int_{1}^{\infty e^{i \phi}}(-\lambda)^{n} \exp (-\lambda t) \hat{r}(\lambda) d \lambda
$$


and

$$
\left|\left(\frac{d}{d t}\right)^{n} R(t)\right| \leq A \int_{0}^{+\infty}|\lambda|^{n} \exp \left(-c_{0}|\lambda|^{\delta}\right) d|\lambda| \leq \frac{A}{c_{0}^{(n+1) / \delta}}\left(\frac{n+1}{\delta}\right) !
$$

The above estimate of $\left(\frac{d}{d t}\right)^{n} R(t)$ means that $R(t) \in \operatorname{Asy}_{\{\gamma\}}(\{t \neq 0 ;|\arg t|<$ $\left.\left.\phi_{0}+\frac{\pi}{2}\right\}\right)$ with $\gamma=\delta /(1-\delta)$. Let us show (2.13). Firstly further assume $\phi_{0} \leq \pi$. Suppose that $\pi / 2<\phi<\phi_{0}$ and $|\arg t+\pi|<\phi-\pi / 2$. Then $-\pi / 2<$ $\arg t+\phi<-3 \pi / 2+2 \phi$ and $3 \pi / 2-2 \phi<\arg t+2 \pi-\phi<\pi / 2$. It follows from the assumption $\pi / 2<\phi<\phi_{0} \leq \pi$ that $-\pi / 2<\arg t+\phi<\pi / 2$ and $-\pi / 2<\arg t+2 \pi-\phi<\pi / 2$. Hence

$$
\begin{aligned}
& \left(\frac{d}{d t}\right)^{n}\left(R(t)-R\left(t e^{2 \pi i}\right)\right) \\
= & \frac{1}{2 \pi i}\left(\int_{1}^{\infty e^{i \phi}}(-\lambda)^{n} \exp (-\lambda t) \hat{r}(\lambda) d \lambda-\int_{1}^{\infty e^{-i \phi}}(-\lambda)^{n} \exp (-\lambda t) \hat{r}(\lambda) d \lambda\right) \\
= & \frac{1}{2 \pi i} \int_{\infty e^{-i \phi}}^{\infty e^{i \phi}}(-\lambda)^{n} \exp (-\lambda t) \hat{r}(\lambda) d \lambda
\end{aligned}
$$

and by the decay estimate of $\hat{r}(\lambda)$ it holds that

$$
\lim _{t \rightarrow 0}\left(\frac{d}{d t}\right)^{n}\left(R(t)-R\left(t e^{2 \pi i}\right)\right)=\frac{1}{2 \pi i} \int_{\infty e^{-i \phi}}^{\infty e^{i \phi}}(-\lambda)^{n} \hat{r}(\lambda) d \lambda=0 .
$$

Consequently by (2.14) and Taylor's formula

$$
\left|R(t)-R\left(t e^{2 \pi i}\right)\right| \leq \frac{2 A|t|^{n}}{c_{0}^{(n+1) / \delta} n !}\left(\frac{n+1}{\delta}\right) ! \leq A^{\prime} B^{n}|t|^{n}\left(\frac{n+1}{\gamma}\right) !
$$

for any $n \in \mathbb{N}$. Since $\phi$ is an arbitrary constant with $\pi / 2<\phi<\phi_{0}$, it follows from Lemma 6.1 in Section 6 that there exist positive constants $C$ and $c$ such that (2.13) holds. If $\phi_{0}>\pi$, by considering the rotation $\hat{r}_{\varphi}(\lambda)=\hat{r}\left(\lambda e^{i \varphi}\right)$, we have (2.13).

In the case of $\delta=1$ it follows easily from the decay estimate of $\hat{r}(\lambda)$ that $R(t)$ is holomorphic in $\left\{|t|<c_{0}\right\}$.

Let us define a path $\mathcal{C}_{0}$ in $w_{0}$-space, which appears in the sequel to define integral operators on a sectorial region. $\mathcal{C}_{0}$ is a path which starts at $w_{0}=0$, encloses $w_{0}=z_{0}$ once anticlockwise and ends at $w_{0}=0 . \mathcal{C}_{0}$ depends on $z_{0}$.

Suppose that $\hat{r}(\lambda)$ satisfies the conditions in Lemma 2.4, and the constants $\phi_{0}, c_{0}$ and $\delta$ are those in Lemma 2.4. Let $R(t)$ be a function defined by $(2.12)$ 


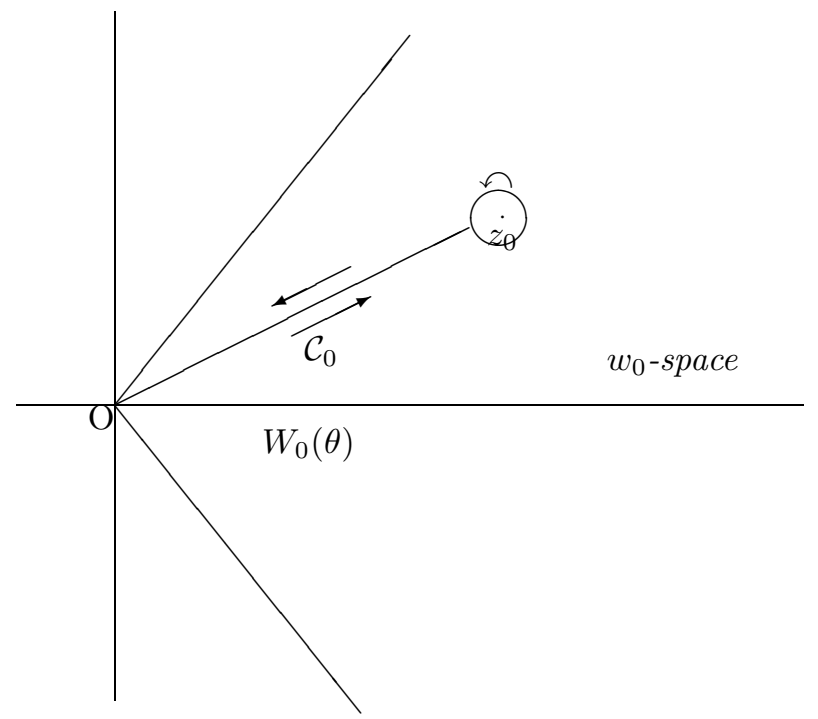

Figure 2. Path $\mathcal{C}_{0}$

and $W_{0}$ be an open disk in $\mathbb{C}$ centered at the origin such that $W_{0} \subset\left\{\left|w_{0}\right|<c_{0}\right\}$. Let $f\left(w_{0}\right) \in \mathcal{O}\left(W_{0}\left(\theta_{0}\right)\right)$ with $0<\theta_{0}<\phi_{0}-\frac{\pi}{2}$ and be bounded. Suppose $z_{0} \in$ $W_{0}\left(\theta_{0}\right)$. Then we may take $\mathcal{C}_{0} \subset W_{0}\left(\theta_{0}\right)$ and $\left|\arg \left(w_{0}-z_{0}\right)\right|<\theta_{0}+\pi<\phi_{0}+\frac{\pi}{2}$ for $w_{0} \in \mathcal{C}_{0}$. We can define

$$
(R f)\left(z_{0}\right)=\frac{1}{2 \pi i} \int_{\mathcal{C}_{0}} R\left(w_{0}-z_{0}\right) f\left(w_{0}\right) d w_{0}
$$

and $(R f)\left(z_{0}\right) \in \mathcal{O}\left(W_{0}\left(\theta_{0}\right)\right)$. In particular if $\delta=1, R(t)$ is holomorphic in a neighborhood of $t=0$, hence $(R f)\left(z_{0}\right)=0$. We have more precisely

Proposition 2.5. Suppose that $\hat{r}(\lambda)$ satisfies the conditions in Lemma 2.4. Let $f\left(z_{0}\right) \in \mathcal{O}\left(W_{0}\left(\theta_{0}\right)\right)\left(0<\theta_{0}<\phi_{0}-\frac{\pi}{2}\right)$ be bounded and $(R f)\left(z_{0}\right)$ be the operator defined by $(2.16)$.

(1) If $\delta=1$, then $(R f)\left(z_{0}\right)=0$.

(2) If $0<\delta<1$, then $(R f)\left(z_{0}\right) \in \mathcal{O}\left(W_{0}\left(\theta_{0}\right)\right)$ and there exist positive constants $A$ and $c$ which are independent of $f\left(w_{0}\right)$ such that

$$
\left|(R f)\left(z_{0}\right)\right| \leq A \exp \left(-c\left|z_{0}\right|^{-\gamma}\right) \sup _{w_{0} \in W_{0}\left(\theta_{0}\right)}\left|f\left(w_{0}\right)\right| .
$$


Proof. We only have to treat the case $0<\delta<1$. $R(t)$ is bounded on $\left\{t \neq 0 ;|\arg t|<\phi_{0}+\frac{\pi}{2}\right\}$ by Lemma 2.4. We can deform the path $\mathcal{C}_{0}$ to the segment jointing $w_{0}=0$ with $w_{0}=z_{0}$ and the infinitesimal small circle with center $w_{0}=z_{0}$ and have

$$
(R f)\left(z_{0}\right)=\int_{0}^{z_{0}}\left(R\left(w_{0}-z_{0}\right)-R\left(\left(w_{0}-z_{0}\right) e^{2 \pi i}\right)\right) f\left(w_{0}\right) d w_{0} .
$$

By Lemma 2.4

$$
\begin{aligned}
\left|(R f)\left(z_{0}\right)\right| & \leq C \sup _{w_{0} \in W\left(\theta_{0}\right)}\left|f\left(w_{0}\right)\right| \int_{0}^{z_{0}} \exp \left(-c\left|z_{0}-w_{0}\right|^{-\gamma}\right)\left|d w_{0}\right| \\
& \leq A \exp \left(-c\left|z_{0}\right|^{-\gamma}\right)\left(\sup _{w_{0} \in W\left(\theta_{0}\right)}\left|f\left(w_{0}\right)\right|\right) .
\end{aligned}
$$

Let us apply Propositions 2.3 and 2.5 and Lemma 2.4 to $R_{p, h, s}(t)$ and $\left(R_{p, h, s} f\right)$ $\left(z_{0}\right)$ (see $(2.7)$ and $\left.(2.11)\right)$.

Proposition 2.6. Let $W_{0}$ be a polydisk centered at the origin and $f\left(w_{0}\right) \in \mathcal{O}\left(W_{0}\left(\theta_{0}\right)\right)$ be bounded. Let $\left(R_{p, h, s} f\right)\left(z_{0}\right)$ be the operator defined by (2.11). If $\delta=1$, then $\left(R_{p, h, s} f\right)\left(z_{0}\right)=0$.

Let $0<\delta<1, \gamma=\delta /(1-\delta)$ and $s_{0} \in \mathbb{N}$. Suppose further $0<\theta_{0}<\pi / 2 \gamma$ and $0 \leq s \leq s_{0}$. Put $h_{-}=\min \{h, 0\}$ and $h_{+}=\max \{h, 0\}$. Then the following estimates hold.

(1) If $p \leq h_{-}$, then $\left(R_{p, h, s} f\right)\left(z_{0}\right) \equiv 0$.

(2) If $h_{-}<p<h_{+}$, then there are positive constants $A=A\left(\theta_{0}\right), B=B\left(\theta_{0}\right)$ and $c=c\left(d, \theta_{0}\right)$ such that

$$
\left|\left(R_{p, h, s} f\right)\left(z_{0}\right)\right| \leq A B^{|h|}(1+|p|)^{s_{0}} d^{\frac{p-h_{+}}{\delta}}\left(\frac{h_{+}-p}{\delta}\right) ! e^{-c\left|z_{0}\right|^{-\gamma}} \sup _{w_{0} \in W_{0}\left(\theta_{0}\right)}\left|f\left(w_{0}\right)\right| .
$$

(3) If $p \geq h_{+}$, then there are positive constants $A=A\left(\theta_{0}\right), B=B\left(\theta_{0}\right)$ and $c=c\left(d, \theta_{0}\right)$ such that

$$
\left|\left(R_{p, h, s} f\right)\left(z_{0}\right)\right| \leq \frac{A B^{|h|}(1+|p|)^{s_{0}} d^{\frac{p-h_{+}}{\delta}} e^{-c\left|z_{0}\right|^{-\gamma}}}{\left(\frac{p-h_{+}}{\delta}\right) !} \sup _{w_{0} \in W_{0}\left(\theta_{0}\right)}\left|f\left(w_{0}\right)\right| .
$$


Proof. Assume $0<\delta<1$. Then we can choose $\phi_{0}$ such that $\theta_{0}+\frac{\pi}{2}<$ $\phi_{0}<\frac{\pi}{2 \delta}$ by $0<\theta_{0}<\frac{\pi}{2 \gamma}=\frac{\pi}{2}\left(\frac{1}{\delta}-1\right)$. The assertions follow from the estimates in Propositions 2.3 and 2.5 and Lemma 2.4.

Finally let us study an integral operator,

$$
\left(K_{p} f\right)\left(z_{0}\right)=\frac{1}{2 \pi i} \int_{\mathcal{C}_{0}} K_{p}\left(\delta ; w_{0}-z_{0}\right) f\left(w_{0}\right) d w_{0}
$$

which also appears in the sequel and depends on $\delta$ and $d$ (see (2.1) and (2.2)).

Proposition 2.7. Let $W_{0}$ be a polydisk centered at the origin and $f\left(w_{0}\right)$ $\in \mathcal{O}\left(W_{0}\left(\theta_{0}\right)\right)$ be bounded. Let $\left(K_{p} f\right)\left(z_{0}\right)$ be the operator defined by $(2.20)$.

(1) If $\delta=1$ and $p \geq 1$, then for $z_{0} \in W_{0}\left(\theta_{0}\right)$ with $\left|z_{0}\right|<d$

$$
\left(K_{p} f\right)\left(z_{0}\right)=\int_{0}^{z_{0}} \frac{\left(z_{0}-w_{0}\right)^{p-1}}{(p-1) !} f\left(w_{0}\right) d w_{0} .
$$

(2) Let $0<\delta<1$ and $\gamma=\delta /(1-\delta)$. Suppose $0<\theta_{0}<\pi / 2 \gamma$. Then $\left(K_{p} f\right)\left(z_{0}\right) \in$ $\mathcal{O}\left(W_{0}\left(\theta_{0}\right)\right)$ and there is a constant $C$ such that for $z_{0} \in W_{0}\left(\theta_{0}\right)$

$$
\left|\left(K_{p} f\right)\left(z_{0}\right)\right| \leq\left\{\begin{array}{l}
C d^{\frac{p-2}{\delta}} \sup _{w_{0} \in W_{0}}\left|f\left(w_{0}\right)\right| /\left(\frac{p}{\delta}\right) ! \quad \text { for } p \geq 2 \\
C \sup _{w_{0} \in W_{0}}\left|f\left(w_{0}\right)\right| \quad \text { for } p=1
\end{array}\right.
$$

(3) Suppose $p \leq 0$. Then

$$
\left(K_{p} f\right)\left(z_{0}\right)=\left(\frac{\partial}{\partial z_{0}}\right)^{|p|} f\left(z_{0}\right) .
$$

Proof. (1) If $\delta=1$, then by Lemma 2.1-(2) for $p \geq 1$

$$
\begin{aligned}
\int_{\mathcal{C}_{0}} K_{p}\left(w_{0}-z_{0}\right) f\left(w_{0}\right) d w_{0} & =\frac{(-1)^{p}}{2 \pi i(p-1) !} \int_{\mathcal{C}_{0}}\left(w_{0}-z_{0}\right)^{p-1} \log \left(w_{0}-z_{0}\right) f\left(w_{0}\right) d w_{0} \\
& =\int_{0}^{z_{0}} \frac{\left(z_{0}-w_{0}\right)^{p-1}}{(p-1) !} f\left(w_{0}\right) d w_{0} .
\end{aligned}
$$

(2) Let $0<\delta<1$ and $z_{0} \in W_{0}\left(\theta_{0}\right)$. By the assumption $0<\theta_{0}<\pi / 2 \gamma$ we can choose $\phi_{0}$ with $\theta_{0}+\frac{\pi}{2}<\phi_{0}<\frac{\pi}{2 \delta}$. Then it holds that $\left|\arg \left(w_{0}-z_{0}\right)\right|<\theta_{0}+\pi<$ $\phi_{0}+\pi / 2$ and (2.22) follows from Lemma 2.1-(1).

(3) We have (2.23) by Lemma 2.1-(3). 


\section{$\S 3 . \quad$ Construction of Parametrix-1}

Let us find $g(z)$ in Theorem 1.2. Let $w=\left(w_{0}, w_{1}, \ldots, w_{d}\right)=\left(w_{0}, w^{\prime}\right) \in$ $\mathbb{C}^{d+1}$. Define

$$
\begin{aligned}
K_{p, q}\left(\delta ; w_{0}-z_{0}, w_{0}\right) & =w_{0}^{q}\left(-\frac{\partial}{\partial w_{0}}\right)^{q} K_{p}\left(\delta ; w_{0}-z_{0}\right) \\
& =\frac{w_{0}^{q}}{2 \pi i} \int_{1}^{\infty} \exp \left(-\lambda\left(w_{0}-z_{0}\right)\right) \lambda^{q} \hat{g}_{p}(\lambda) d \lambda .
\end{aligned}
$$

We note $K_{p, 0}\left(\delta ; w_{0}-z_{0}, w_{0}\right)=K_{p}\left(\delta ; w_{0}-z_{0}\right)$.

We assume condition $\left(\mathrm{C}_{i}\right)$ for fixed $i$ with $0 \leq i \leq p^{*}-1$. Put $\delta=\delta_{i}:=$ $\gamma_{i} /\left(\gamma_{i}+1\right)$ in the definition of $K_{p}(\delta ; t)$. We denote simply $K_{p}(\delta ; t)$ by $K_{p}(t)$ and $K_{p, q}\left(\delta ; w_{0}-z_{0}, w_{0}\right)$ by $K_{p, q}\left(w_{0}-z_{0}, w_{0}\right)$ respectively. Let $W=W_{0} \times W^{\prime}$ be a small open polydisk centered at the origin in $\mathbb{C}^{d+1}$ and $f(w) \in \operatorname{Asy}_{\{\gamma\}}(W(\theta))$ with $\gamma_{i+1} \leq \gamma<\gamma_{i}$. We try to find $g(z)$ in Theorem 1.2 in the following form:

$$
\begin{aligned}
g(z) & =\sum_{p \in \mathbb{Z}, q \in \mathbb{N}}\left(G_{p, q} f\right)(z) \\
\left(G_{p, q} f\right)(z) & =\int_{\mathcal{C}} k_{p, q}\left(z, w^{\prime}\right) K_{p, q}\left(w_{0}-z_{0}, w_{0}\right) f\left(w_{0}, w^{\prime}\right) d w \\
& =\int_{\mathcal{C}^{\prime}} k_{p, q}\left(z, w^{\prime}\right) d w^{\prime} \int_{\mathcal{C}_{0}} K_{p, q}\left(w_{0}-z_{0}, w_{0}\right) f\left(w_{0}, w^{\prime}\right) d w_{0},
\end{aligned}
$$

where the path $\mathcal{C}=\mathcal{C}_{0} \times \mathcal{C}^{\prime}$ is defined as follows. $\mathcal{C}_{0}$ is the one in the sectorial region $W_{0}(\theta)$ defined in Section 2 (see Figure 2) and $\mathcal{C}^{\prime}$ is a chain in $\mathbb{C}^{d}$ defined by the $d$-dimensional product of circles $\prod_{h=1}^{d}\left\{\left|w_{i}\right|=r_{1}\right\}$. Then $\mathcal{C}=\mathcal{C}_{0} \times \mathcal{C}^{\prime}$ is a $(d+1)$-dimensional chain in $\mathbb{C}^{d+1}$. Functions $\left\{k_{p, q}\left(z, w^{\prime}\right)\right\}_{p \in \mathbb{Z}, q \in \mathbb{N}}$ which are holomorphic in $\left(z, w^{\prime}\right)$ in a neighborhood of $\{z=0\} \times\left\{w^{\prime} \in \mathcal{C}^{\prime}\right\}$ and they will be determined in order that $g(z)$ has the properties stated in Theorem 1.2. Put

$$
(G f)(z)=\sum_{p \in \mathbb{Z}, q \in \mathbb{N}}\left(G_{p, q} f\right)(z)
$$

and we call $G$ a parametrix. The main purpose in this section is to show how to determine $k_{p, q}\left(z, w^{\prime}\right)$. The convergence and the properties of the operator $G$ are studied in the following sections.

First we give some lemmas and use the notation $\partial_{\lambda}=\frac{\partial}{\partial \lambda}$. 
Lemma 3.1. The following identity holds:

$$
\begin{aligned}
& z_{0}^{j} \partial_{z_{0}}^{h} \int_{\mathcal{C}_{0}} K_{p, q}\left(w_{0}-z_{0}, w_{0}\right) f\left(w_{0}, w^{\prime}\right) d w_{0} \\
& =\int_{\mathcal{C}_{0}}\left(\int_{1}^{\infty}\left(\partial_{\lambda}^{j} e^{\lambda z_{0}}\right) e^{-\lambda w_{0}} \lambda^{q+h} \hat{g}_{p}(\lambda) d \lambda\right) w_{0}^{q} f\left(w_{0}, w^{\prime}\right) d w_{0} .
\end{aligned}
$$

We have easily (3.4) by differentiating (3.1).

Lemma 3.2. $\quad$ There exist constants $C_{j, a, s, s^{\prime}}$ such that

$$
\left(-\partial_{\lambda}\right)^{j}\left(e^{-\lambda w_{0}} \lambda^{a} \hat{g}(\lambda)\right)=e^{-\lambda w_{0}} \lambda^{-j+a}\left(\sum_{s+s^{\prime} \leq j} C_{j, a, s, s^{\prime}}\left(\lambda w_{0}\right)^{s^{\prime}}\left(-\lambda \partial_{\lambda}\right)^{s} \hat{g}(\lambda)\right)
$$

and

$$
\left|C_{j, a, s, s^{\prime}}\right| \leq A(1+|a|)^{j-s-s^{\prime}}
$$

for a constant $A=A(j)$ depending only on $j$.

Proof. If $j=0,(3.5)$ is obvious. We have inductively

$$
\begin{aligned}
& \left(-\partial_{\lambda}\right)^{j+1}\left(e^{-\lambda w_{0}} \lambda^{a} \hat{g}(\lambda)\right) \\
& =\left(-\partial_{\lambda}\right)\left(e^{-\lambda w_{0}} \lambda^{-j+a}\left(\sum_{s+s^{\prime} \leq j} C_{j, a, s, s^{\prime}}\left(\lambda w_{0}\right)^{s^{\prime}}\left(-\lambda \partial_{\lambda}\right)^{s} \hat{g}(\lambda)\right)\right) \\
& =e^{-\lambda w_{0}} \lambda^{-j-1+a}\left(\sum _ { s + s ^ { \prime } \leq j } \left(C_{j, a, s, s^{\prime}}\left(\lambda w_{0}\right)^{s^{\prime}+1}\left(-\lambda \partial_{\lambda}\right)^{s} \hat{g}(\lambda)+\left(j-a-s^{\prime}\right)\right.\right. \\
& \left.\left.\quad \times C_{j, a, s, s^{\prime}}\left(\lambda w_{0}\right)^{s^{\prime}}\left(-\lambda \partial_{\lambda}\right)^{s} \hat{g}(\lambda)+C_{j, a, s, s^{\prime}}\left(\lambda w_{0}\right)^{s^{\prime}}\left(-\lambda \partial_{\lambda}\right)^{s+1} \hat{g}(\lambda)\right)\right)
\end{aligned}
$$

Hence $C_{j+1, a, s, s^{\prime}}=C_{j, a, s, s^{\prime}-1}+\left(j-a-s^{\prime}\right) C_{j, a, s, s^{\prime}}+C_{j, a, s-1, s^{\prime}}$ holds and we have (3.6) by induction. 
We remark that the constants $C_{j, a, s, s^{\prime}}\left(s+s^{\prime} \leq j\right)$ will often appear.

Lemma 3.3. The following identity holds:

$$
\begin{aligned}
& z_{0}^{j} \partial_{z_{0}}^{h} \int_{\mathcal{C}_{0}} K_{p, q}\left(w_{0}-z_{0}, w_{0}\right) f\left(w_{0}, w^{\prime}\right) d w_{0} \\
& =\sum_{s+s^{\prime} \leq j} C_{j, q+h, s, s^{\prime}}\left(\int_{\mathcal{C}_{0}} p^{s} K_{p+j-h, q+s^{\prime}}\left(w_{0}-z_{0}, w_{0}\right) f\left(w_{0}, w^{\prime}\right) d w_{0}\right. \\
& \left.\quad+\int_{\mathcal{C}_{0}} R_{p,-j+h, s}\left(w_{0}-z_{0}\right) \partial_{w_{0}}^{q+s^{\prime}}\left(w_{0}^{q+s^{\prime}} f\left(w_{0}, w^{\prime}\right)\right) d w_{0}\right) .
\end{aligned}
$$

Proof. We have from Lemma 3.1 and by integrations by parts

$$
\begin{aligned}
& z_{0}^{j} \partial_{z_{0}}^{h} \int_{\mathcal{C}_{0}} K_{p, q}\left(w_{0}-z_{0}, w_{0}\right) f\left(w_{0}, w^{\prime}\right) d w_{0} \\
& =\int_{\mathcal{C}_{0}}\left(\int_{1}^{\infty}\left(\partial_{\lambda}^{j} e^{\lambda z_{0}}\right) e^{-\lambda w_{0}} \lambda^{q+h} \hat{g}_{p}(\lambda) d \lambda\right) w_{0}^{q} f\left(w_{0}, w^{\prime}\right) d w_{0} \\
& =\int_{\mathcal{C}_{0}}\left(-\int_{1}^{\infty}\left(\partial_{\lambda}^{j-1} e^{\lambda z_{0}}\right) \partial_{\lambda}\left(e^{-\lambda w_{0}} \lambda^{q+h} \hat{g}_{p}(\lambda)\right) d \lambda\right) w_{0}^{q} f\left(w_{0}, w^{\prime}\right) d w_{0} \\
& +\int_{\mathcal{C}_{0}}\left(\text { an entire function in } w_{0}\right) \times f\left(w_{0}, w^{\prime}\right) d w_{0} \\
& =\int_{\mathcal{C}_{0}}\left(\int_{1}^{\infty} e^{\lambda z_{0}}\left(-\partial_{\lambda}\right)^{j}\left(e^{-\lambda w_{0}} \lambda^{q+h} \hat{g}_{p}(\lambda)\right) d \lambda\right) w_{0}^{q} f\left(w_{0}, w^{\prime}\right) d w_{0} \\
& =\int_{\mathcal{C}_{0}} w_{0}^{q} f\left(w_{0}, w^{\prime}\right) d w_{0}\left(\int_{1}^{\infty} e^{-\lambda\left(w_{0}-z_{0}\right)} \lambda^{q-j+h}\right. \\
& \left.\times\left(\sum_{s+s^{\prime} \leq j} C_{j, q+h, s, s^{\prime}}\left(\lambda w_{0}\right)^{s^{\prime}}\left(-\lambda \partial_{\lambda}\right)^{s} \hat{g}_{p}(\lambda)\right) d \lambda\right) \\
& =\sum_{s+s^{\prime} \leq j} C_{j, q+h, s, s^{\prime}} \int_{\mathcal{C}_{0}} w_{0}^{q+s^{\prime}} f\left(w_{0}, w^{\prime}\right) d w_{0} \int_{1}^{\infty} e^{-\lambda\left(w_{0}-z_{0}\right)} \lambda^{q-j+h+s^{\prime}} \\
& \times\left(-\lambda \partial_{\lambda}\right)^{s} \hat{g}_{p}(\lambda) d \lambda \\
& =\sum_{s+s^{\prime} \leq j} C_{j, q+h, s, s^{\prime}} \int_{\mathcal{C}_{0}} w_{0}^{q+s^{\prime}} f\left(w_{0}, w^{\prime}\right) d w_{0} \\
& \times\left(-\partial_{w_{0}}\right)^{q+s^{\prime}} \int_{1}^{\infty} e^{-\lambda\left(w_{0}-z_{0}\right)} \lambda^{-j+h}\left(-\lambda \partial_{\lambda}\right)^{s} \hat{g}_{p}(\lambda) d \lambda .
\end{aligned}
$$


We have from Lemma 2.2

$$
\begin{aligned}
& \int_{1}^{\infty} e^{-\lambda\left(w_{0}-z_{0}\right)} \lambda^{-j+h}\left(-\lambda \partial_{\lambda}\right)^{s} \hat{g}_{p}(\lambda) d \lambda \\
& =p^{s} K_{p+j-h}\left(w_{0}-z_{0}\right)+R_{p,-j+h, s}\left(w_{0}-z_{0}\right),
\end{aligned}
$$

hence

$$
\begin{aligned}
z_{0}^{j} \partial_{z_{0}}^{h} & \int_{\mathcal{C}_{0}} K_{p, q}\left(w_{0}-z_{0}, w_{0}\right) f\left(w_{0}, w^{\prime}\right) d w_{0} \\
= & \sum_{s+s^{\prime} \leq j} C_{j, q+h, s, s^{\prime}}\left(\int_{\mathcal{C}_{0}}\left(-\partial_{w_{0}}\right)^{q+s^{\prime}} p^{s} K_{p+j-h}\left(w_{0}-z_{0}\right) w_{0}^{q+s^{\prime}} f\left(w_{0}, w^{\prime}\right) d w_{0}\right. \\
& \left.\quad+\int_{\mathcal{C}_{0}}\left(-\partial_{w_{0}}\right)^{q+s^{\prime}} R_{p,-j+h, s}\left(w_{0}-z_{0}\right) w_{0}^{q+s^{\prime}} f\left(w_{0}, w^{\prime}\right) d w_{0}\right) \\
= & \sum_{s+s^{\prime} \leq j} C_{j, q+h, s, s^{\prime}}\left(\int_{\mathcal{C}_{0}} p^{s} K_{p+j-h, q+s^{\prime}}\left(w_{0}-z_{0}, w_{0}\right) f\left(w_{0}, w^{\prime}\right) d w_{0}\right. \\
& \left.+\int_{\mathcal{C}_{0}} R_{p,-j+h, s}\left(w_{0}-z_{0}\right) \partial_{w_{0}}^{q+s^{\prime}}\left(w_{0}^{q+s^{\prime}} f\left(w_{0}, w^{\prime}\right)\right) d w_{0}\right) .
\end{aligned}
$$

Proposition 3.4. It holds that

$$
\begin{aligned}
& z_{0}^{j_{\alpha}} b_{\alpha}(z) \partial_{z}^{\alpha} \int_{\mathcal{C}^{\prime}} k_{p, q}\left(z, w^{\prime}\right) d w^{\prime} \int_{\mathcal{C}_{0}} K_{p, q}\left(w_{0}-z_{0}, w_{0}\right) f\left(w_{0}, w^{\prime}\right) d w_{0} \\
& =\sum_{\substack{0 \leq l \leq \alpha_{0} \\
s+s^{\prime} \leq j_{\alpha}}} b_{\alpha, l}(z) C_{j_{\alpha}, q+\alpha_{0}-l, s, s^{\prime}} \int_{\mathcal{C}^{\prime}} \partial_{z^{\prime}}^{\alpha^{\prime}} \partial_{z_{0}}^{l} k_{p, q}\left(z, w^{\prime}\right) d w^{\prime} \\
& \quad \times \int_{\mathcal{C}_{0}} p^{s} K_{p+e_{\alpha}+l, q+s^{\prime}}\left(w_{0}-z_{0}, w_{0}\right) f\left(w_{0}, w^{\prime}\right) d w_{0}+\left(R_{\alpha, p, q}^{\#} f\right)(z),
\end{aligned}
$$

where $b_{\alpha, l}(z)=\left(\begin{array}{c}\alpha_{0} \\ l\end{array}\right) b_{\alpha}(z)$ and

$$
\begin{aligned}
\left(R_{\alpha, p, q}^{\#} f\right)(z)= & \sum_{\substack{0 \leq l \leq \alpha_{0} \\
s+s^{\prime} \leq j_{\alpha}}} C_{j_{\alpha}, q+\alpha_{0}-l, s, s^{\prime}} b_{\alpha, l}(z) \int_{\mathcal{C}^{\prime}} \partial_{z^{\prime}}^{\alpha^{\prime}} \partial_{z_{0}}^{l} k_{p, q}\left(z, w^{\prime}\right) d w^{\prime} \\
& \times \int_{\mathcal{C}_{0}} R_{p,-e_{\alpha}-l, s}\left(w_{0}-z_{0}\right) \partial_{w_{0}}^{q+s^{\prime}}\left(w_{0}^{q+s^{\prime}} f\left(w_{0}, w^{\prime}\right)\right) d w_{0}
\end{aligned}
$$


Proof. By Leibniz' formula and Lemma 3.3 and putting $b_{\alpha, l}(z)=\left(\begin{array}{c}\alpha_{0} \\ l\end{array}\right)$ $b_{\alpha}(z)$,

$$
\begin{aligned}
& z_{0}^{j_{\alpha}} b_{\alpha}(z) \partial_{z}^{\alpha} \int_{\mathcal{C}^{\prime}} k_{p, q}\left(z, w^{\prime}\right) d w^{\prime} \int_{\mathcal{C}_{0}} K_{p, q}\left(w_{0}-z_{0}, w_{0}\right) f\left(w_{0}, w^{\prime}\right) d w_{0} \\
& =\sum_{l=0}^{\alpha_{0}} b_{\alpha, l}(z) \int_{\mathcal{C}^{\prime}} \partial_{z^{\prime}}^{\alpha^{\prime}} \partial_{z_{0}}^{l} k_{p, q}\left(z, w^{\prime}\right) d w^{\prime}\left(z_{0}^{j_{\alpha}} \partial_{z_{0}}^{\alpha_{0}-l} \int_{\mathcal{C}_{0}} K_{p, q}\left(w_{0}-z_{0}, w_{0}\right) f\left(w_{0}, w^{\prime}\right) d w_{0}\right) \\
& =\sum_{l=0}^{\alpha_{0}} b_{\alpha, l} \int_{\mathcal{C}^{\prime}} \partial_{z^{\prime}}^{\alpha^{\prime}} \partial_{z_{0}}^{l} k_{p, q}\left(z, w^{\prime}\right) d w^{\prime}\left(\sum_{s+s^{\prime} \leq j_{\alpha}} C_{j_{\alpha}, q+\alpha_{0}-l, s, s^{\prime}}\right. \\
& \quad \times\left(\int_{\mathcal{C}_{0}} p^{s} K_{p+j_{\alpha}-\alpha_{0}+l, q+s^{\prime}}\left(w_{0}-z_{0}, w_{0}\right) f\left(w_{0}, w^{\prime}\right) d w_{0}\right. \\
& \left.\left.\quad+\int_{\mathcal{C}_{0}} R_{p,-j_{\alpha}+\alpha_{0}-l, s}\left(w_{0}-z_{0}\right) \partial_{w_{0}}^{q+s^{\prime}}\left(w_{0}^{q+s^{\prime}} f\left(w_{0}, w^{\prime}\right)\right) d w_{0}\right)\right)
\end{aligned}
$$

By $e_{\alpha}=j_{\alpha}-\alpha_{0}$, we have (3.9) and (3.10).

Now let us show how to construct a parametrix $G$ (see (3.2) and (3.3)). We assume condition $\left(\mathrm{C}_{i}\right)$ for some fixed $i$. Define for $0 \leq h \leq e(i)-$ $e\left(p^{*}-1\right)$

$$
\left\{\begin{array}{l}
P_{0}\left(z, \partial_{z}\right)=\sum_{\left\{\alpha ; e_{\alpha} \geq e(i)\right\}} z_{0}^{j_{\alpha}} b_{\alpha}(z) \partial_{z}^{\alpha}, \\
P_{h}\left(z, \partial_{z}\right)=\sum_{\left\{\alpha ; e_{\alpha}-e(i)=-h\right\}} z_{0}^{j_{\alpha}} b_{\alpha}(z) \partial_{z}^{\alpha} \quad \text { for } \quad h \geq 1 .
\end{array}\right.
$$

$P_{h}\left(z, \partial_{z}\right)$ depends on $i$. Then we have a decomposition of $P\left(z, \partial_{z}\right), P\left(z, \partial_{z}\right)=$ $\sum_{h=0}^{e(i)-e\left(p^{*}-1\right)} P_{h}\left(z, \partial_{z}\right)$ depending on $i$. In particular $P\left(z, \partial_{z}\right)=P_{0}\left(z, \partial_{z}\right)$ if $i=p^{*}-1$. We construct $G$ by a successive approximation. Find $G^{n}(n \in \mathbb{N})$ so as to satisfy

$$
\left\{\begin{array}{l}
\left(P_{0}\left(z, \partial_{z}\right)\left(G^{0} f\right)(z)-f(z)\right) \sim 0 \\
P_{0}\left(z, \partial_{z}\right)\left(G^{n} f\right)(z)+\sum_{h=1}^{e(i)-e\left(p^{*}-1\right)} P_{h}\left(z, \partial_{z}\right)\left(G^{n-h} f\right)(z) \sim 0
\end{array}\right.
$$


where $\sim 0$ means zero asymptotic expansion in $\operatorname{Asy}_{\left\{\gamma_{i}\right\}}\left(U\left(\theta^{\prime}\right)\right)$ for some polydisk $U$ and $0<\theta^{\prime}<\theta$. Then we may expect that $G=\sum_{n=0}^{\infty} G^{n}$ satisfies $(P(z, \partial)(G f)(z)-f(z)) \sim 0$ and is a desired parametrix. Set

$$
\begin{aligned}
\left(G^{n} f\right)(z) & =\int_{\mathcal{C}} G^{n}(z, w) f(w) d w \\
G^{n}(z, w) & =\sum_{\substack{p \geq p_{n} \\
q \geq 0}} k_{p, q}^{n}\left(z, w^{\prime}\right) K_{p, q}\left(w_{0}-z_{0}, w_{0}\right) .
\end{aligned}
$$

It is the main aim to obtain equations that determine the coefficients $k_{p, q}^{n}\left(z, w^{\prime}\right)$ of $G^{n}(z, w)$ and to solve them. We have formally from Proposition 3.4

$$
\begin{aligned}
& P_{0}\left(z, \partial_{z}\right)\left(G^{n} f\right)=\sum_{\left\{\alpha ; e_{\alpha} \geq e(i)\right\}} \sum_{\substack{p^{\prime} \geq p_{n} \\
q^{\prime} \geq 0}}\left\{\left(\sum_{\substack{0 \leq l \leq \alpha_{0} \\
s+s^{\prime} \leq j_{\alpha}}} b_{\alpha, l}(z) C_{j_{\alpha}, q^{\prime}+\alpha_{0}-l, s, s^{\prime}}\right.\right. \\
& \left.\times \int_{\mathcal{C}^{\prime}} \partial_{z^{\prime}}^{\alpha^{\prime}} \partial_{z_{0}}^{l} k_{p^{\prime}, q^{\prime}}^{n}\left(z, w^{\prime}\right) d w^{\prime} p^{\prime s} \int_{\mathcal{C}_{0}} K_{p^{\prime}+e_{\alpha}+l, q^{\prime}+s^{\prime}}\left(w_{0}-z_{0}, w_{0}\right) f\left(w_{0}, w^{\prime}\right) d w_{0}\right) \\
& \left.+\left(R_{\alpha, p^{\prime}, q^{\prime}}^{\#} f\right)(z)\right\} \\
& =\sum_{\substack{p \geq p_{n}+e(i) \\
q \geq 0}}\left(\sum_{\substack{\left(\alpha, l, s, s^{\prime}, p^{\prime}, q^{\prime}\right) ; \\
p^{\prime}+e_{\alpha}+l=p, q^{\prime}+s^{\prime}=q \\
e_{\alpha}-e(i) \geq 0,0 \leq l \leq \alpha_{0} \\
s+s^{\prime} \leq j_{\alpha}}} C_{j_{\alpha}, q^{\prime}+\alpha_{0}-l, s, s^{\prime}} p^{\prime s}\right. \\
& \left.\times \int_{\mathcal{C}^{\prime}} b_{\alpha, l}(z) \partial_{z^{\prime}}^{\alpha^{\prime}} \partial_{z_{0}}^{l} k_{p^{\prime}, q^{\prime}}^{n}\left(z, w^{\prime}\right) d w^{\prime}\right) \\
& \times \int_{\mathcal{C}_{0}} K_{p, q}\left(w_{0}-z_{0}, w_{0}\right) f\left(w_{0}, w^{\prime}\right) d w_{0}+\sum_{\{\alpha ; e(\alpha) \geq e(i)\}} \sum_{\substack{p \geq p_{n} \\
q \geq 0}}\left(R_{\alpha, p, q}^{\#} f\right)(z),
\end{aligned}
$$

where we sum up firstly $\left(\alpha, l, s, s^{\prime}, p^{\prime}, q^{\prime}\right)$ satisfying $p^{\prime}+e_{\alpha}+l=p$ and $q^{\prime}+s^{\prime}=q$ for given $p, q$. 
We also have in the same way

$$
\begin{aligned}
P_{h}\left(z, \partial_{z}\right)\left(G^{n-h} f\right)= & \sum_{\substack{p \geq p_{n-h}+e(i)-h \\
q \geq 0}}\left(\sum_{\substack{\left(\alpha, l, s, s^{\prime}, p^{\prime}, q^{\prime}\right) ; \\
p^{\prime}+e_{\alpha}+l=p, q^{\prime}+s^{\prime}=q \\
e_{\alpha}-e(i)=-h, 0 \leq l \leq \alpha_{0} \\
s+s^{\prime} \leq j_{\alpha}}} C_{j_{\alpha}, q^{\prime}+\alpha_{0}-l, s, s^{\prime}} p^{s}\right. \\
& \left.\times \int_{\mathcal{C}^{\prime}} b_{\alpha, l}(z) \partial_{z^{\prime}}^{\alpha^{\prime}} \partial_{z_{0}}^{l} k_{p^{\prime}, q^{\prime}}^{n-h}\left(z, w^{\prime}\right) d w^{\prime}\right) \int_{\mathcal{C}_{0}} K_{p, q}\left(w_{0}-z_{0}, w_{0}\right) \\
& \times f\left(w_{0}, w^{\prime}\right) d w_{0}+\sum_{\left\{\alpha ; e_{\alpha}-e(i)=-h\right\}} \sum_{\substack{p \geq p_{n} \\
q \geq 0}}\left(R_{\alpha, p, q}^{\#} f\right)(z) .
\end{aligned}
$$

By putting

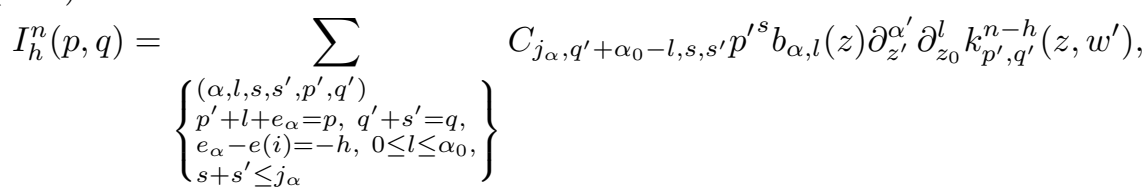

which is a holomorphic function in $z$ and $w^{\prime}$, we have

$$
\begin{aligned}
& P_{h}\left(z, \partial_{z}\right)\left(G^{n-h} f\right) \sum_{\substack{p \geq p_{n-h}+e(i)-h \\
q \geq 0}} \int_{\mathcal{C}^{\prime}} I_{h}^{n}(p, q) d w^{\prime} \int_{\mathcal{C}_{0}} K_{p, q}\left(w_{0}-z_{0}, w_{0}\right) f\left(w_{0}, w^{\prime}\right) d w_{0} \\
& \quad+\sum_{\left\{\alpha ; e_{\alpha}-e(i)=-h\right\}} \sum_{\substack{p \geq p_{n} \\
q \geq 0}}\left(R_{\alpha, p, q}^{\#} f\right)(z) .
\end{aligned}
$$

On the other hand, by $K_{0,0}\left(w_{0}-z_{0}, w_{0}\right)=\frac{1}{2 \pi i\left(w_{0}-z_{0}\right)}+$ an entire function,

$$
f(z)=\frac{1}{(2 \pi i)^{d}} \int_{\mathcal{C}^{\prime}} d w^{\prime} \int_{C_{0}} \frac{K_{0,0}\left(w_{0}-z_{0}, w_{0}\right) f\left(w_{0}, w^{\prime}\right)}{\prod_{j=1}^{d}\left(w_{j}-z_{j}\right)} d w_{0} .
$$

Thus by considering the first relation in (3.11) and (3.12) and by comparing the coefficients of $K_{p, q}\left(w_{0}-z_{0}, w_{0}\right)$, we have equations that determine 
$k_{p, q}^{0}\left(z, w^{\prime}\right):$

$$
\begin{gathered}
\sum_{\left\{\begin{array}{l}
\left(\alpha, l, s, s^{\prime}, p^{\prime}, q^{\prime}\right) ; \\
p^{\prime}+e_{\alpha}+l=p, q^{\prime}+s^{\prime}=q \\
e_{\alpha}-e(i) \geq 0,0 \leq l \leq \alpha_{0} \\
s+s^{\prime} \leq j_{\alpha}
\end{array}\right\}} C_{j_{\alpha}, q^{\prime}+\alpha_{0}-l, s, s^{\prime}} p^{s} b_{\alpha, l}(z) \partial_{z^{\prime}}^{\alpha^{\prime}} \partial_{z_{0}}^{l} k_{p^{\prime}, q^{\prime}}^{0}\left(z, w^{\prime}\right) \\
=\frac{\delta_{p, 0} \delta_{q, 0}}{(2 \pi i)^{d} \prod_{j=1}^{d}\left(w_{j}-z_{j}\right)}
\end{gathered}
$$

where we omit the terms $\left(R_{\alpha, p, q}^{\#} f\right)(z)$ in (3.12), because by Proposition 2.6 and (3.10) they decay exponentially with order $\gamma_{i}$ as $z_{0}$ tends to 0 in some sectorial region. Similarly we have from the second relation in (3.11) and (3.14)

$$
\begin{array}{ll}
\sum_{\left\{\begin{array}{l}
\left(\alpha, l, s, s^{\prime}, p^{\prime}, q^{\prime}\right) ; \\
p^{\prime}+e_{\alpha}+l=p, q^{\prime}+s^{\prime}=q \\
e_{\alpha}-e(i) \geq 0,0 \leq l \leq \alpha_{0} \\
s+s^{\prime} \leq j_{\alpha}
\end{array}\right\}} & C_{j_{\alpha}, q^{\prime}+\alpha_{0}-l, s, s^{\prime}} p^{s} b_{\alpha, l}(z) \partial_{z^{\prime}}^{\alpha^{\prime}} \partial_{z_{0}}^{l} k_{p^{\prime}, q^{\prime}}^{n}\left(z, w^{\prime}\right) \\
& +\sum_{h=1}^{e(i)-e\left(p^{*}-1\right)} I_{h}^{n}(p, q)=0 .
\end{array}
$$

We decompose the first sum in (3.17) (the left hand side of (3.16)) into two parts. One is the sum with respect to $\left(\alpha, l, s, s^{\prime}, p^{\prime}, q^{\prime}\right)$ with $e_{\alpha}=e(i)$ and $l=s^{\prime}=0$, hence $p^{\prime}=p-e(i), q^{\prime}=q$. Set

$$
\mathcal{P}\left(p, q ; z, \partial_{z^{\prime}}\right)=\sum_{\left\{(\alpha, s) ; e_{\alpha}=e(i), 0 \leq s \leq j_{\alpha}\right\}} C_{j_{\alpha}, q+\alpha_{0}, s, 0}(p-e(i))^{s} b_{\alpha, 0}(z) \partial_{z^{\prime}}^{\alpha^{\prime}}
$$

The other is the rest denoted by $I_{0}^{n}(p, q)$, that is, the sum of the terms satisfying $l+e_{\alpha}-e(i)+s^{\prime}>0$

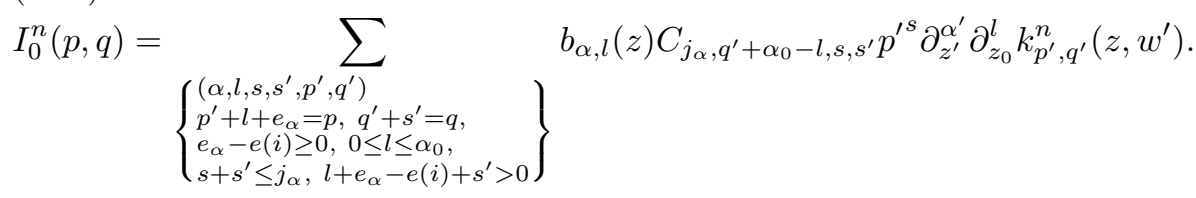


Then it follows from the above decomposition that the equations (3.16) and (3.17) are written as follows:

$$
\begin{aligned}
& \mathcal{P}\left(p, q ; z, \partial_{z^{\prime}}\right) k_{p-e(i), q}^{n}\left(z, w^{\prime}\right)+I_{0}^{n}(p, q)+\sum_{h=1}^{e(i)-e\left(p^{*}-1\right)} I_{h}^{n}(p, q) \\
& =\frac{\delta_{n, 0} \delta_{p, 0} \delta_{q, 0}}{(2 \pi i)^{d} \prod_{j=1}^{d}\left(w_{j}-z_{j}\right)} .
\end{aligned}
$$

Here we note that $I_{0}^{n}(p, q)$ is determined by $k_{p^{\prime} \cdot q^{\prime}}^{n}\left(z, w^{\prime}\right)$ with $p^{\prime}+q^{\prime}<p+q-e(i)$ and $I_{h}^{n}(p, q)$ is determined by $k_{p^{\prime} \cdot q^{\prime}}^{n-h}\left(z, w^{\prime}\right)$.

We show that (3.20) is solvable under the condition $\left(\mathrm{C}_{i}\right)$. We have $\Delta_{0}(i)=$ $\left\{\alpha=\left(k_{i}-l_{i}, \alpha^{\prime}\right) ;\left|\alpha^{\prime}\right|=l_{i}, j_{\alpha}=0\right\}$ and $e(i)=-k_{i}+l_{i} \leq 0$. Since $\chi_{P, i}\left(0, \xi^{\prime}\right) \not \equiv 0$, we may assume $\chi_{P, i}\left(0, \hat{\xi}^{\prime}\right) \neq 0$ for $\hat{\xi}^{\prime}=(1,0, \ldots, 0) \in \mathbb{C}^{d}$, that is, $b_{\alpha(i)}(0) \neq 0$ for $\alpha(i)=\left(k_{i}-l_{i}, l_{i}, 0, \ldots, 0\right) \in \mathbb{N}^{d+1}$. Hence further we may assume $b_{\alpha(i)}(z)=$ 1. $\mathcal{P}\left(p, q ; z, \partial_{z^{\prime}}\right)$ is a partial differential operator with order $l_{i}$. It depends on parameters $p$ and $q$, however, its principal part $\sum_{\alpha \in \Delta_{0}(i)} b_{\alpha}(z) \partial_{z^{\prime}}^{\alpha^{\prime}}$ does not. By the assumption $b_{\alpha(i)}(0) \neq 0, \mathcal{P}\left(p, q ; z, \partial_{z^{\prime}}\right)$ is noncharacteristic with respect to $\left\{z_{1}=0\right\}$. Hence we can consider the Cauchy problem for the equation (3.20). Let $n=0$. Then we have the following Cauchy problems for $k_{p, q}^{0}\left(z, w^{\prime}\right)$ $\left(p \geq p_{0}=-e(i), q \geq 0\right)$ :

$$
\left\{\begin{array}{l}
\mathcal{P}\left(0,0 ; z, \partial_{z^{\prime}}\right) k_{-e(i), 0}^{0}\left(z, w^{\prime}\right)=\frac{1}{(2 \pi i)^{d} \prod_{j=1}^{d}\left(w_{j}-z_{j}\right)} \\
\partial_{z_{1}}^{l} k_{-e(i), 0}^{0}\left(z_{0}, 0, z^{\prime \prime}, w^{\prime}\right)=0 \quad \text { for } \quad 0 \leq l \leq l_{i}-1,
\end{array}\right.
$$

and for $(p, q) \neq(-e(i), 0)$

$$
\left\{\begin{array}{c}
\mathcal{P}\left(p+e(i), q ; z, \partial_{z^{\prime}}\right) k_{p, q}^{0}\left(z, w^{\prime}\right)+I_{0}^{0}(p+e(i), q)=0 \\
\partial_{z_{1}}^{h} k_{p, q}^{0}\left(z_{0}, 0, z^{\prime \prime}, w^{\prime}\right)=0 \quad \text { for } \quad 0 \leq h \leq l_{i}-1 .
\end{array}\right.
$$

Suppose that the coefficients of $P\left(z, \partial_{z}\right)$ are holomorphic in $\left\{z ;\left|z_{i}\right| \leq\right.$ $R, 0 \leq i \leq d\}$. Let $0<r_{0}<r_{1}<r_{2}<R$ and $\left|z_{i}\right| \leq r_{0}<r_{1} \leq\left|w_{i}\right| \leq r_{2}$ for $1 \leq i \leq d$. Then we can define $k_{-e(i), 0}^{0}\left(z, w^{\prime}\right)$ by $(3.21)$. As for $(p, q) \neq$ $(-e(i), 0), I_{0}^{0}(p+e(i), q)$ is determined by $\left\{k_{p^{\prime}, q^{\prime}}^{0}\left(z, w^{\prime}\right) ; p^{\prime}+q^{\prime}<p+q\right\}$. So we can solve the equation (3.22) successively. Therefore we obtain $k_{p, q}^{0}\left(z, w^{\prime}\right)(p \geq$ $\left.p_{0}=-e(i), q \geq 0\right)$ in a neighborhood of $z=0$.

Next let $n \geq 1$ and consider the Cauchy Problem

$$
\left\{\begin{array}{l}
\mathcal{P}\left(p, q ; z, \partial_{z^{\prime}}\right) k_{p-e(i), q}^{n}\left(z, w^{\prime}\right)+I_{0}^{n}(p, q)+\sum_{h=1}^{e(i)-e\left(p^{*}-1\right)} I_{h}^{n}(p . q)=0 \\
\partial_{z_{1}}^{h} k_{p, q}^{n}\left(z_{0}, 0, z^{\prime \prime}, w^{\prime}\right)=0 \quad \text { for } \quad 0 \leq h \leq l_{i}-1 .
\end{array}\right.
$$


Since $I_{h}^{n}(p, q)(h \geq 1)$ is determined by $k_{p^{\prime}, q^{\prime}}^{n-h}\left(z, w^{\prime}\right)$, we have $p_{n}=-e(i)-n$ and can solve (3.23) successively. Consequently $\left\{k_{p, q}^{n}\left(z, w^{\prime}\right) ; n \in \mathbb{N}, p \geq p_{n}, q \geq 0\right\}$ are determined in a neighborhood of $z=0$.

We give formal relations concerning $k_{p, q}^{n}\left(z, w^{\prime}\right)$. Set

$$
k_{p, q}\left(z, w^{\prime}\right)=\sum_{n=\max \{0,-p-e(i)\}}^{+\infty} k_{p, q}^{n}\left(z, w^{\prime}\right) .
$$

In particular $k_{p, q}\left(z, w^{\prime}\right)=k_{p, q}^{0}\left(z, w^{\prime}\right)$ if $i=p^{*}-1$. Then we have formally

$$
\begin{aligned}
G(z, w) & =\sum_{n=0}^{+\infty} G^{n}(z, w)=\sum_{n=0}^{+\infty} \sum_{p=p_{n}}^{+\infty} \sum_{q=0}^{+\infty} k_{p, q}^{n}\left(z, w^{\prime}\right) K_{p, q}\left(w_{0}-z_{0}, w_{0}\right) \\
& =\sum_{p=-\infty}^{+\infty} \sum_{q=0}^{+\infty} k_{p, q}\left(z, w^{\prime}\right) K_{p, q}\left(w_{0}-z_{0}, w_{0}\right) .
\end{aligned}
$$

We give another formal relation of $\left\{k_{p, q}\left(z, w^{\prime}\right) ; p \in \mathbb{Z}, q \in \mathbb{N}\right\}$

$$
\begin{aligned}
& \sum_{\left.\begin{array}{l}
\left(\alpha, l, s, s^{\prime}, p^{\prime}, q^{\prime}\right) \\
p^{\prime}+l+e_{\alpha}=p, q^{\prime}+s^{\prime}=q, \\
0 \leq l \leq \alpha_{0}, s+s^{\prime} \leq j_{\alpha}
\end{array}\right\}} b_{\alpha, l}(z) C_{j_{\alpha}, q^{\prime}+\alpha_{0}-l, s, s^{\prime}} p^{\prime s} \partial_{z^{\prime}}^{\alpha^{\prime}} \partial_{z_{0}}^{l} k_{p^{\prime}, q^{\prime}}\left(z, w^{\prime}\right) \\
= & \frac{\delta_{p, 0} \delta_{q, 0}}{(2 \pi i)^{d} \prod_{j=1}^{d}\left(w_{j}-z_{j}\right)}
\end{aligned}
$$

which is available to show that $G$ is a parametrix. Let us show how to obtain (3.26) formally. From (3.16) and (3.17) we have for $n \in \mathbb{N}$

$$
\begin{aligned}
& \begin{array}{l}
\sum_{\left\{\begin{array}{l}
\left(\alpha, l, s, s^{\prime}, p^{\prime}, q^{\prime}\right) \\
p^{\prime}+l+e_{\alpha}=p, q^{\prime}+s^{\prime}=q, \\
e_{\alpha}-e(i) \geq 0,0 \leq l \leq \alpha_{0}, \\
s+s^{\prime} \leq j_{\alpha}
\end{array}\right\}} b_{\alpha, l}(z) C_{j_{\alpha}, q^{\prime}+\alpha_{0}-l, s, s^{\prime}} p^{\prime s} \partial_{z^{\prime}}^{\alpha^{\prime}} \partial_{z_{0}}^{l} k_{p^{\prime}, q^{\prime}}^{n}\left(z, w^{\prime}\right) \\
e^{(i)-e\left(p^{*}-1\right)}
\end{array} \\
& +\sum_{h=1}^{e(i)-e\left(p^{*}-1\right)} \sum_{\substack{\left(\alpha, l, s, s^{\prime}, p^{\prime}, q^{\prime}\right) \\
p^{\prime}+l+e_{\alpha}=p, q^{\prime}+s^{\prime}=q, e_{\alpha}-e(i)=-h, 0 \leq l \leq \alpha_{0}, s+s^{\prime} \leq j_{\alpha}}} b_{\alpha, l}(z) C_{j_{\alpha}, q^{\prime}+\alpha_{0}-l, s, s^{\prime}} p^{s} \partial_{z^{\prime}}^{\alpha^{\prime}} \partial_{z_{0}}^{l} k_{p^{\prime}, q^{\prime}}^{n-h}\left(z, w^{\prime}\right) \\
& =\frac{\delta_{n, 0} \delta_{p, 0} \delta_{q, 0}}{(2 \pi i)^{d} \prod_{j=1}^{d}\left(w_{j}-z_{j}\right)} .
\end{aligned}
$$


By summing up (3.27) formally with respect to $n$, we have

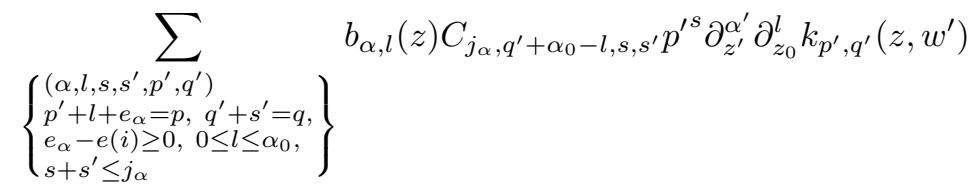

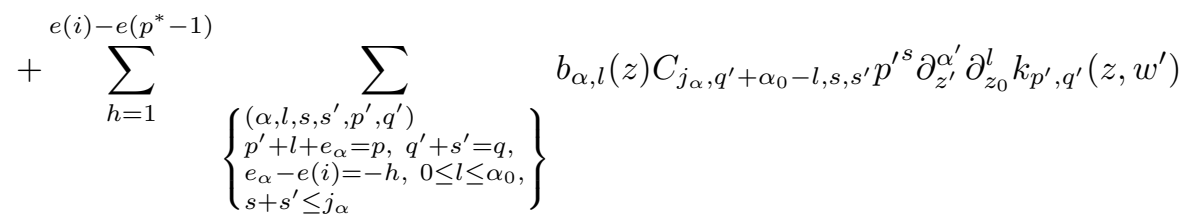

$$
\begin{aligned}
& =\frac{\delta_{p, 0} \delta_{q, 0}}{(2 \pi i)^{d} \prod_{j=1}^{d}\left(w_{j}-z_{j}\right)},
\end{aligned}
$$

which implies (3.26). We show in the following section that $k_{p, q}\left(z, w^{\prime}\right)$ converges (Proposition 4.2). Therefore the relation (3.26) is analytically valid.

\section{$\S 4$. Construction of Parametrix-2}

In this section firstly we show the convergence of $k_{p, q}\left(z, w^{\prime}\right)$ (see (3.24)) and define an integral operator $(G f)(z)$,

$$
\begin{aligned}
(G f)(z) & :=\sum_{p=-\infty}^{+\infty} \sum_{q=0}^{\infty}\left(G_{p, q} f\right)(z) \\
\left(G_{p, q} f\right)(z) & :=\int_{\mathcal{C}^{\prime}} k_{p, q}\left(z, w^{\prime}\right) d w^{\prime} \int_{\mathcal{C}_{0}} K_{p, q}\left(w_{0}-z_{0}, w_{0}\right) f\left(w_{0}, w^{\prime}\right) d w_{0} .
\end{aligned}
$$

Secondly we study the properties of $g(z):=(G f)(z)$ and $(R f)(z):=P\left(z, \partial_{z}\right)$ $g(z)-f(z)$, and show Theorems 1.2 and 1.3. As in the previous section we assume condition $\left(\mathrm{C}_{i}\right)$ for a fixed $i, 0 \leq i \leq p^{*}-1$ and suppose that the coefficients of $P\left(z, \partial_{z}\right)$ are holomorphic in $\left\{z \in \mathbb{C}^{d+1} ;\left|z_{i}\right| \leq R, 0 \leq i \leq d\right\}$ and let $r_{i}(0 \leq i \leq 2)$ be positive constants with $r_{0}<r_{1}<r_{2}<R$. First of all let us estimate $k_{p, q}^{n}\left(z, w^{\prime}\right)$. Recall $\delta_{i}=\gamma_{i} /\left(\gamma_{i}+1\right)$.

Theorem 4.1. Suppose that $\left|z_{i}\right| \leq r_{0}<r_{1} \leq\left|w_{i}\right| \leq r_{2}$ for $1 \leq i \leq d$. Then there exist constants $A, B, C, \rho_{0} \geq 1$ and a small $r>0$ such that for $\left|z_{0}\right|+\rho\left|z_{1}\right|+\sum_{i=2}^{d}\left|z_{i}\right| \leq r / 2$ and $\rho \geq \rho_{0}$ the following estimates hold. 


$$
\text { If } 0 \leq i \leq p^{*}-2 \text {, then } k_{p, q}^{n}\left(z, w^{\prime}\right)=0 \text { for } p<-e(i)-n \text { and }
$$

$$
\left|\partial_{z}^{\alpha} k_{p, q}^{n}\left(z, w^{\prime}\right)\right| \leq A^{n+1} B^{p+e(i)+n+q}(\rho C)^{|\alpha|} \frac{\rho^{(p+e(i)+n) / \delta_{i}}}{\rho^{n+q}} \frac{\left(\frac{p+e(i)+n}{\delta_{i}}\right) !|\alpha| !}{\left(\frac{n}{\delta_{i+1}}\right) ! q !}
$$

for $p \geq-e(i)-n$.

$$
\text { If } i=p^{*}-1 \text {, then } k_{p, q}^{0}\left(z, w^{\prime}\right)=0 \text { for } p<-e\left(p^{*}-1\right) \text { and }
$$

$$
\left|\partial_{z}^{\alpha} k_{p, q}^{0}\left(z, w^{\prime}\right)\right| \leq A B^{p+e\left(p^{*}-1\right)+q}(\rho C)^{|\alpha|} \frac{\rho^{\left(p+e\left(p^{*}-1\right)\right) / \delta_{i}}}{\rho^{q}} \frac{\left(\frac{p+e\left(p^{*}-1\right)}{\delta_{i}}\right) !|\alpha| !}{q !}
$$

for $p \geq-e\left(p^{*}-1\right)$. Here constants $A, B$ and $C$ are independent of $\rho$.

The proof of Theorem 4.1 is given in Section 6 . We note that if $i=p^{*}-1$, then $k_{p, q}^{n}\left(z, w^{\prime}\right)=0$ for $n \geq 1$ and $k_{p, q}\left(z, w^{\prime}\right)=k_{p, q}^{0}\left(z, w^{\prime}\right)$. Let us show the convergence of $k_{p, q}\left(z, w^{\prime}\right)=\sum_{n=\max \{0,-p-e(i)\}}^{+\infty} k_{p, q}^{n}\left(z, w^{\prime}\right)$.

Proposition 4.2. Let $\left|z_{0}\right|+\rho\left|z_{1}\right|+\sum_{i=2}^{d}\left|z_{i}\right| \leq r / 2$. Then there are constants $A_{\rho}$ depending on $\rho$ with $\rho \geq \rho_{0}, B$ and $C$ such that

$$
\left|\partial_{z}^{\alpha} k_{p, q}\left(z, w^{\prime}\right)\right| \leq\left\{\begin{array}{l}
\frac{A_{\rho} B^{q}\left(\rho^{1 / \delta_{i}} B\right)^{p}(\rho C)^{|\alpha|}\left(\frac{p}{\delta_{i}}\right) !|\alpha| !}{\rho^{q} q !} \text { for } p \geq 0, \\
\frac{A_{\rho} A^{|p|} B^{q}(\rho C)^{|\alpha|}|\alpha| !}{\rho^{q+|p|} q !\left(\frac{|p|}{\delta_{i+1}}\right) !} \text { for } i \neq p^{*}-1 \text { and } p \leq 0,
\end{array}\right.
$$

where $\rho_{0}$ is that in Theorem 4.1 .

Proof. Assume $i \neq p^{*}-1$. Let $p+e(i) \geq 0$. Then by Theorem 4.1 we have

$$
\begin{aligned}
& \left|\partial_{z}^{\alpha} k_{p, q}\left(z, w^{\prime}\right)\right| \leq \sum_{n=0}^{+\infty}\left|\partial_{z}^{\alpha} k_{p, q}^{n}\left(z, w^{\prime}\right)\right| \\
& \leq \frac{A B^{q}\left(\rho^{1 / \delta_{i}} B\right)^{p+e(i)}(\rho C)^{|\alpha|}|\alpha| !}{\rho^{q} q !} \sum_{n=0}^{+\infty} \frac{\left(\rho^{1 / \delta_{i}} A B\right)^{n}}{\rho^{n}} \frac{\left(\frac{p+e(i)+n}{\delta_{i}}\right) !}{\left(\frac{n}{\delta_{i+1}}\right) !} \\
& \leq \frac{A_{\rho}^{\prime} B^{q}\left(\rho^{1 / \delta_{i}} B\right)^{p+e(i)}(\rho C)^{|\alpha|}|\alpha| ! C_{0}^{p+e(i)}\left(\frac{p+e(i)}{\delta_{i}}\right) !}{\rho^{q} q !}
\end{aligned}
$$


Let $p+e(i)<0$. Then by Theorem 4.1 we have

$$
\begin{aligned}
\left|\partial_{z}^{\alpha} k_{p, q}\left(z, w^{\prime}\right)\right| & \leq \sum_{n=-p-e(i)}^{+\infty}\left|\partial_{z}^{\alpha} k_{p, q}^{n}\left(z, w^{\prime}\right)\right| \\
& \leq \frac{A^{-p-e(i)} B^{q}(\rho C)^{|\alpha|}|\alpha| !}{\rho^{q-p-e(i)} q !} \sum_{n=0}^{+\infty} \frac{(A B)^{n} \rho^{n / \delta_{i}}\left(\frac{n}{\delta_{i}}\right) !}{\rho^{n}\left(\frac{n-p-e(i)}{\delta_{i+1}}\right) !} \\
& \leq \frac{A^{|p+e(i)|} B^{q}(\rho C)^{|\alpha|}|\alpha| !}{\rho^{q+|p+e(i)|} q !\left(\frac{|p+e(i)|}{\delta_{i+1}}\right) !} \sum_{n=0}^{+\infty} \frac{(A B)^{n} \rho^{n / \delta_{i}}\left(\frac{n}{\delta_{i}}\right) !}{\rho^{n}\left(\frac{n}{\delta_{i+1}}\right) !} \\
& \leq \frac{A_{\rho}^{\prime} A^{|p+e(i)|} B^{q}(\rho C)^{|\alpha|}|\alpha| !}{\rho^{q+|p+e(i)|} q !\left(\frac{|p+e(i)|}{\delta_{i+1}}\right) !}
\end{aligned}
$$

The estimate (4.3) follows from the above two inequalities with other constants $A_{\rho}, B$ and $C$. If $i=p^{*}-1$, the estimate easily follows from (4.2b).

Let $W$ be a small open polydisk centered at the origin and $f(z) \in \mathcal{O}(W(\theta))$ be bounded. Define as in the previous sections (see (3.2))

$$
\begin{aligned}
\left(G_{p, q} f\right)(z) & =\int_{\mathcal{C}} k_{p, q}\left(z, w^{\prime}\right) K_{p, q}\left(w_{0}-z_{0}, w_{0}\right) f(w) d w \\
& =\int_{\mathcal{C}^{\prime}} k_{p, q}\left(z, w^{\prime}\right) d w^{\prime} \int_{\mathcal{C}_{0}} K_{p, q}\left(w_{0}-z_{0}, w_{0}\right) f\left(w_{0}, w^{\prime}\right) d w_{0} .
\end{aligned}
$$

Lemma 4.3. $\quad$ Suppose that $f(w) \in \mathcal{O}(W(\theta))$ is bounded. Then

$$
\begin{aligned}
& \int_{\mathcal{C}_{0}} K_{p, q}\left(w_{0}-z_{0}, w_{0}\right) f\left(w_{0}, w^{\prime}\right) d w_{0} \\
& =\int_{\mathcal{C}_{0}} K_{p}\left(w_{0}-z_{0}\right)\left(\frac{\partial}{\partial w_{0}}\right)^{q}\left(w_{0}^{q} f\left(w_{0}, w^{\prime}\right)\right) d w_{0}
\end{aligned}
$$

and in particular if $i=0$ and $p>0$,

$$
\begin{aligned}
\int_{\mathcal{C}_{0}} & K_{p, q}\left(w_{0}-z_{0}, w_{0}\right) f\left(w_{0}, w^{\prime}\right) d w_{0} \\
\quad= & \int_{0}^{z_{0}} \frac{\left(z_{0}-w_{0}\right)^{p-1}}{(p-1) !}\left(\frac{\partial}{\partial w_{0}}\right)^{q}\left(w_{0}^{q} f\left(w_{0}, w^{\prime}\right)\right) d w_{0} .
\end{aligned}
$$

Proof. Since $f(z)$ is bounded on $W(\theta)$, we have (4.5) by integrations by parts. If $i=0$ and $p>0$, then $\delta_{0}=1$ and we have (4.6) by Proposition 2.7. 
By Lemma $4.3\left(G_{p, q} f\right)(z)$ is of the form

$$
\left(G_{p, q} f\right)(z)=\int_{\mathcal{C}^{\prime}} k_{p, q}\left(z, w^{\prime}\right) d w^{\prime} \int_{\mathcal{C}_{0}} K_{p}\left(w_{0}-z_{0}\right)\left(\frac{\partial}{\partial w_{0}}\right)^{q}\left(w_{0}^{q} f\left(w_{0}, w^{\prime}\right)\right) d w_{0}
$$

and $\left(G_{p, q} f\right)(z) \in \mathcal{O}(W(\theta))$.

Put $(G f)(z):=\sum_{p \in \mathbb{Z}, q \in \mathbb{N}}\left(G_{p, q} f\right)(z)$. Our concerns are to show the convergence of $(G f)(z)$, to estimate its derivatives and to show that $g(z)=(G f)(z)$ has the properties stated in Theorem 1.2, that is, $G$ is a parametrix of $P\left(z, \partial_{z}\right)$. We need estimates of $\partial_{w_{0}}^{q}\left(w_{0}^{q} f\left(w_{0}, w^{\prime}\right)\right)$ and $\partial_{w_{0}}^{n+q}\left(w_{0}^{q} f\left(w_{0}, w^{\prime}\right)\right)$ for our purposes.

Lemma 4.4. Suppose $f(w) \in \mathcal{O}(W(\theta))$. Let $0<\theta_{0}<\theta$ and put $M=$ $\sup \left\{\left|f\left(w_{0}, w^{\prime}\right)\right| ; w \in W\left(\theta_{0}\right)\right\}$. Let $V$ be a polydisk with $V \Subset W$ and $\eta>0$ be an arbitrary small constant. Then

$$
\left|\left(\frac{\partial}{\partial w_{0}}\right)^{q} w_{0}^{q} f\left(w_{0}, w^{\prime}\right)\right| \leq \frac{M C_{0}^{q} q !}{(\sin \eta)^{q}} \quad \text { for } z \in V\left(\theta_{0}-\eta\right),
$$

where $C_{0}$ is independent of $f(w)$ and $\eta$.

Further assume $f(w) \in \operatorname{Asy}_{\{\gamma\}}(W(\theta))$. If $\gamma>0$ and $\left|\partial_{w_{0}}^{n} f(w)\right| \leq M F^{n}$ $(n / \delta) !(\delta=\gamma /(\gamma+1))$ for $z \in W\left(\theta_{0}\right)$, then

$$
\left|\left(\frac{\partial}{\partial w_{0}}\right)^{n+q} w_{0}^{q} f\left(w_{0}, w^{\prime}\right)\right| \leq \frac{M F_{*}^{n} C_{0}^{q} q !}{(\sin \eta)^{q}}\left(\frac{n}{\delta}\right) ! \quad \text { for } z \in V\left(\theta_{0}-\eta\right),
$$

and if $\gamma=0$ and $\left|\partial_{w_{0}}^{n} f(w)\right| \leq M_{n}$ for $z \in W\left(\theta_{0}\right)$, then

$$
\left|\left(\frac{\partial}{\partial w_{0}}\right)^{n+q} w_{0}^{q} f\left(w_{0}, w^{\prime}\right)\right| \leq \frac{M_{n} C_{1}^{n} C_{0}^{q} q !}{(\sin \eta)^{q}} \quad \text { for } z \in V\left(\theta_{0}-\eta\right),
$$

where $C_{0}$ and $C_{1}$ are independent of $f(w)$ and $\eta$, and $F_{*}$ does not depend on $\eta$ but depends on the constant $F$ appearing in the bounds of $\partial_{w_{0}}^{n} f(w)$.

Proof. Let $z \in V\left(\theta_{0}-\eta\right)$. Then by Cauchy's integral formula

$$
\left(\frac{\partial}{\partial w_{0}}\right)^{q} w_{0}^{q} f(w)=\frac{q !}{2 \pi i} \oint_{\mathcal{Z}} \frac{\zeta^{q} f\left(\zeta, w^{\prime}\right) d \zeta}{\left(\zeta-w_{0}\right)^{q+1}}
$$


where $\mathcal{Z}$ is a circle $\zeta-w_{0}=\left|w_{0}\right| \sin (\eta / 2) e^{i \varphi}(0 \leq \varphi \leq 2 \pi)$. We have $|\zeta| \leq 2\left|w_{0}\right|$ on $\mathcal{Z}$, hence

$$
\left|\left(\frac{\partial}{\partial w_{0}}\right)^{q} w_{0}^{q} f(w)\right| \leq \frac{M q !}{2 \pi\left(\left|w_{0}\right| \sin (\eta / 2)\right)^{q}} \int_{0}^{2 \pi}|\zeta|^{q} d \varphi \leq \frac{M C_{0}^{q} q !}{(\sin \eta)^{q}} .
$$

Before proving (4.8), we note that $\left|w_{0}^{q}\left(\frac{\partial}{\partial w_{0}}\right)^{q} f(w)\right| \leq M C_{0}^{q} q ! /(\sin \eta)^{q}$ holds by the same method. Suppose $f(w) \in \operatorname{Asy}_{\{\gamma\}}(W(\theta))$ with $\gamma>0$ and $\left|\partial_{w_{0}}^{n} f(w)\right| \leq$ $M F^{n}\left(\frac{n}{\delta}\right)$ ! on $W\left(\theta_{0}\right)$. Then by the above remark

$$
\left|w_{0}^{s} \partial_{w_{0}}^{s+n} f(w)\right| \leq M F^{n}\left(\frac{n}{\delta}\right) ! C_{0}^{s} s ! /(\sin \eta)^{s} .
$$

We have $\partial_{w_{0}}^{n+q} w^{q} f(w)=\sum_{h=0}^{q}\left(\begin{array}{c}n+q \\ h\end{array}\right) \frac{q !}{(q-h) !} w_{0}^{q-h} \partial_{w_{0}}^{n+q-h} f(w)$ by Leibniz' formula, therefore

$$
\begin{aligned}
& \left|\partial_{w_{0}}^{n+q} w^{q} f(w)\right| \leq M F^{n}\left(\frac{n}{\delta}\right) ! q ! \sum_{h=0}^{q}\left(\begin{array}{c}
n+q \\
h
\end{array}\right) C_{0}^{q-h} /(\sin \eta)^{q-h} \\
\leq & \frac{M F^{n} C_{0}^{q}\left(1+C_{0}^{-1}\right)^{n+q}\left(\frac{n}{\delta}\right) ! q !}{(\sin \eta)^{q}} . \leq \frac{M F^{n} C_{1}^{n+q}\left(\frac{n}{\delta}\right) ! q !}{(\sin \eta)^{q}}
\end{aligned}
$$

This means that (4.8) holds for $F_{*}=C_{1} F$ and another $C_{0}$. We can show (4.9) for $f(w) \in \operatorname{Asy}_{\{0\}}(W(\theta))$ by the same way.

Now let us proceed to show the convergence of $(G f)(z)$ and to obtain the bounds of its derivatives $\partial_{z_{0}}^{n}(G f)(z)$. Let $f(z) \in \mathcal{O}(W(\theta))$ be bounded. We have in the same way as Proposition 3.4 , by putting $\alpha=(n, 0, \ldots, 0), j_{\alpha}=0$ and $b_{\alpha}(z) \equiv 1$,

$$
\begin{aligned}
& \partial_{z_{0}}^{n} \int_{\mathcal{C}^{\prime}} k_{p, q}\left(z, w^{\prime}\right) d w^{\prime} \int_{\mathcal{C}_{0}} K_{p, q}\left(w_{0}-z_{0}, w_{0}\right) f\left(w_{0}, w^{\prime}\right) d w_{0} \\
= & \sum_{l=0}^{n}\left(G_{n, l, p, q} f\right)(z)+\left(R_{n, p, q}^{*} f\right)(z),
\end{aligned}
$$

where

$$
\begin{aligned}
\left(G_{n, l, p, q} f\right)(z) & :=\left(\begin{array}{c}
n \\
l
\end{array}\right) \int_{\mathcal{C}^{\prime}} \partial_{z_{0}}^{l} k_{p, q}\left(z, w^{\prime}\right) d w^{\prime} \\
& \times \int_{\mathcal{C}_{0}} K_{p-n+l}\left(w_{0}-z_{0}\right) \partial_{w_{0}}^{q}\left(w_{0}^{q} f\left(w_{0}, w^{\prime}\right)\right) d w_{0}
\end{aligned}
$$


and

$$
\begin{aligned}
\left(R_{n, p, q}^{*} f\right)(z) & :=\sum_{l=0}^{n}\left(\begin{array}{l}
n \\
l
\end{array}\right) \int_{\mathcal{C}^{\prime}} \partial_{z_{0}}^{l} k_{p, q}\left(z, w^{\prime}\right) d w^{\prime} \\
& \times \int_{\mathcal{C}_{0}} R_{p, n-l, 0}\left(w_{0}-z_{0}\right) \partial_{w_{0}}^{q}\left(w_{0}^{q} f\left(w_{0}, w^{\prime}\right)\right) d w_{0}
\end{aligned}
$$

We note that $\left(R_{n, p, q}^{*} f\right)(z) \equiv 0$ for $p \leq 0$. Define $I_{n, p}$ by

$$
\begin{aligned}
I_{n, p}= & \sum_{\substack{q \in \mathbb{N} \\
0 \leq l \leq n}}\left(\begin{array}{l}
n \\
l
\end{array}\right) \int_{\mathcal{C}^{\prime}} \partial_{z_{0}}^{l} k_{p+n-l, q}\left(z, w^{\prime}\right) d w^{\prime} \\
& \times \int_{\mathcal{C}_{0}} K_{p}\left(w_{0}-z_{0}\right) \partial_{w_{0}}^{q}\left(w_{0}^{q} f\left(w_{0}, w^{\prime}\right)\right) d w_{0} .
\end{aligned}
$$

Then we have

$$
\begin{aligned}
\partial_{z_{0}}^{n}(G f)(z) & =\sum_{\substack{p \in \mathbb{Z}, q \in \mathbb{N} \\
0 \leq l \leq n}}\left(G_{n, l, p, q} f\right)(z)+\sum_{p \in \mathbb{Z}, q \in \mathbb{N}}\left(R_{n, p, q}^{*} f\right)(z) \\
& =\sum_{p \in \mathbb{Z}} I_{n, p}+\sum_{p \in \mathbb{N}, q \in \mathbb{N}}\left(R_{n, p, q}^{*} f\right)(z),
\end{aligned}
$$

provided the above sums converge.

Proposition 4.5. Suppose $p^{*} \geq 2, i \in\left\{1,2, \ldots, p^{*}-1\right\}$ and $f(w) \in$ $\operatorname{Asy}_{\{\gamma\}}(W(\theta))$ with $\gamma_{i+1} \leq \gamma<\gamma_{i}$. Let $0<\theta^{\prime}<\min \left\{\theta, \pi / 2 \gamma_{i}\right\}$. Then there is a polydisk $U$ such that $(G f)(z)$ converges on $U\left(\theta^{\prime}\right)$ and $(G f)(z) \in \operatorname{Asy}_{\{\gamma\}}\left(U\left(\theta^{\prime}\right)\right)$. Moreover if $f(w) \sim 0$, then $(G f)(z) \sim 0$.

Proposition 4.5 is obtained by estimating $I_{n, p}$ and $\left(R_{n, p, q}^{*} f\right)(z)$. The dependence of the polydisk follows from the following proof and we comment it in Theorems 4.7 and 4.8 .

Proof. Firstly we give the proof for $1 \leq i<p^{*}-1$ and secondly for $i=p^{*}-1$.

(1) Suppose $1 \leq i<p^{*}-1$. Then $0=\gamma_{p^{*}}<\gamma_{i+1} \leq \gamma$. So $0<\delta=$ $\gamma /(\gamma+1)<1$. Let $\theta_{0}$ be a constant with $\theta^{\prime}<\theta_{0}<\min \left\{\theta, \pi / 2 \gamma_{i}\right\}$ and $0<\eta<$ $\theta_{0}-\theta^{\prime}$. Then there are constants $M$ and $F$ such that $\left|\partial_{w_{0}}^{n} f(w)\right| \leq M F^{n}\left(\frac{n}{\delta}\right)$ ! for $z \in W\left(\theta_{0}\right)$. Let $V$ be a small polydisk centered at the origin with $V \Subset W$ such that estimates (4.3) in Proposition 4.2 and the estimates in Lemma 4.4 hold for $z \in V\left(\theta_{0}-\eta\right)$. We study the convergence of $\sum_{p \in \mathbb{Z}} I_{n, p}$ and $\sum_{p, q \in \mathbb{N}}\left(R_{n, p, q}^{*} f\right)(z)$. 
Convergence of $\sum_{p \in \mathbb{Z}} I_{n, p}$. Let $p \geq 0$. Then by choosing $\rho \geq 2 B C_{0} / \sin \eta, B$ and $C$ with $B>C$, it follows from (4.3) and (4.7) that

$$
\begin{aligned}
& \left|I_{n, p}\right| \leq \sum_{q=0}^{\infty} \sum_{l=0}^{n} \frac{A_{\rho} M\left(B C_{0}\right)^{q}}{(\rho \sin \eta)^{q}} \frac{\left(\rho^{1 / \delta_{i}} B\right)^{p+n-l}(\rho C)^{l}\left(\frac{p+n-l}{\delta_{i}}\right) !}{(n-l) !} \\
& \times \int_{\mathcal{C}_{0}}\left|K_{p}\left(w_{0}-z_{0}\right)\right|\left|d w_{0}\right| \leq 2 A_{\rho} M\left(\rho^{1 / \delta_{i}} B\right)^{p+n} \sum_{l=0}^{n}\left(\frac{C}{B \rho^{1 / \delta_{i}-1}}\right)^{l} \frac{n !\left(\frac{p+n-l}{\delta_{i}}\right) !}{(n-l) !} \\
& \times \int_{\mathcal{C}_{0}}\left|K_{p}\left(w_{0}-z_{0}\right)\right|\left|d w_{0}\right| \leq 2 A_{\rho} M\left(\rho^{1 / \delta_{i}} B\right)^{p+n}\left(\frac{p+n}{\delta_{i}}\right) ! \sum_{l=0}^{n}\left(\frac{C}{B}\right)^{l} \\
& \times \int_{\mathcal{C}_{0}}\left|K_{p}\left(w_{0}-z_{0}\right)\right|\left|d w_{0}\right| \leq A_{\rho}^{\prime} M\left(\rho^{1 / \delta_{i}} B\right)^{p+n}\left(\frac{p+n}{\delta_{i}}\right) ! \\
& \times \int_{\mathcal{C}_{0}}\left|K_{p}\left(w_{0}-z_{0}\right)\right|\left|d w_{0}\right| .
\end{aligned}
$$

Let $p \leq 0$. Then it holds that $K_{p}\left(w_{0}-z_{0}\right)=\frac{1}{2 \pi i} \frac{|p| !}{\left(w_{0}-z_{0}\right)^{|p|+1}}$ and

$$
\int_{\mathcal{C}_{0}} K_{p}\left(w_{0}-z_{0}\right) \partial_{w_{0}}^{q}\left(w_{0}^{q} f\left(w_{0}, w^{\prime}\right)\right) d w_{0}=\left(\frac{\partial}{\partial z_{0}}\right)^{|p|+q}\left(z_{0}^{q} f\left(z_{0}, w^{\prime}\right)\right) .
$$

If $-n<p \leq 0$, then by (4.3) and (4.8) and by choosing $\rho \geq 2 B C_{0} / \sin \eta, B$ and $C$ with $B>C$,

$$
\begin{aligned}
\left|I_{n, p}\right| \leq & \sum_{q=0}^{\infty}\left(\sum_{0 \leq l<p+n} A_{\rho} B^{q}\left(\rho^{1 / \delta_{i}} B\right)^{p+n-l}(\rho C)^{l}\left(\frac{p+n-l}{\delta_{i}}\right) ! \frac{n ! M C_{0}^{q} F_{*}^{|p|}\left(\frac{|p|}{\delta}\right) !}{(n-l) !(\rho \sin \eta)^{q}}\right. \\
& \left.+\sum_{p+n \leq l \leq n} \frac{A_{\rho} A^{l-p-n} B^{q}(\rho C)^{l}}{\rho^{l-p-n}\left(\frac{l-p-n}{\delta_{i+1}}\right) !} \frac{n ! M C_{0}^{q} F_{*}^{|p|}\left(\frac{|p|}{\delta}\right) !}{(n-l) !(\rho \sin \eta)^{q}}\right) \\
\leq & 2 A_{\rho} M F_{*}^{|p|}\left(\frac{|p|}{\delta}\right) !\left(B \rho^{1 / \delta_{i}}\right)^{p+n}\left(\sum_{0 \leq l<p+n} \frac{\left(\frac{C}{B}\right)^{l} n !\left(\frac{p+n-l}{\delta_{i}}\right) !}{(n-l) !}\right) \\
& +\frac{2 A_{\rho} M A^{-p-n} F_{*}^{|p|}\left(\frac{|p|}{\delta}\right) !}{\rho^{-p-n}}\left(\sum_{p+n \leq l \leq n} \frac{(A C)^{l} n !}{\left(\frac{l-p-n}{\delta_{i+1}}\right) !(n-l) !}\right) \\
\leq & A_{\rho}^{\prime} M F_{*}^{|p|} B_{1}^{n}\left(\frac{|p|}{\delta}\right) !\left(B \rho^{1 / \delta_{i}}\right)^{p+n}\left(\frac{p+n}{\delta_{i}}\right) ! \\
& +\frac{A_{\rho}^{\prime} M F_{*}^{|p|} C_{1}^{|p|+n}\left(\frac{|p|}{\delta}\right) ! n !}{\rho^{-p-n}|p| !} .
\end{aligned}
$$


If $p \leq-n$, then we have in the same way as preceding

$$
\begin{aligned}
\left|I_{n, p}\right| & \leq \sum_{q=0}^{\infty} \sum_{l=0}^{n} \frac{A_{\rho} A^{l-p-n} B^{q} C^{l}}{\rho^{-p-n}\left(\frac{l-p-n}{\delta_{i+1}}\right) !} \frac{n ! M C_{0}^{q} F_{*}^{|p|}\left(\frac{|p|}{\delta}\right) !}{(n-l) !(\rho \sin \eta)^{q}} \\
& \leq \frac{2 A_{\rho} M A^{-p-n} F_{*}^{p}\left(\frac{|p|}{\delta}\right) !}{\rho^{-p-n}} \sum_{l=0}^{n} \frac{n !(A C)^{l}}{\left(\frac{l-p-n}{\delta_{i+1}}\right) !(n-l) !} \\
& \leq \frac{2 A_{\rho} M A^{-p-n} F_{*}^{|p|}\left(\frac{|p|}{\delta}\right) !}{\rho^{-p-n}\left(\frac{-p-n}{\delta_{i+1}}\right) !} \sum_{l=0}^{n} \frac{n !(A C)^{l}}{l !(n-l) !} \leq \frac{A_{\rho}^{\prime} M F_{*}^{|p|} C_{1}^{n+|p|}\left(\frac{|p|}{\delta}\right) !}{\rho^{-p-n}\left(\frac{-p-n}{\delta_{i+1}}\right) !}
\end{aligned}
$$

Hence by the above bounds for $I_{n, p}$ and Proposition 2.7-(2) we have

$$
\begin{aligned}
& \sum_{p \in \mathbb{Z}}\left|I_{n, p}\right| \leq A_{\rho}^{\prime} M\left(\sum_{p \leq-n} \frac{F_{*}^{|p|} C_{1}^{n+|p|}\left(\frac{|p|}{\delta}\right) !}{\rho^{-p-n}\left(\frac{-p-n}{\delta_{i+1}}\right) !}\right. \\
& +\sum_{-n<p<0}\left(F_{*}^{|p|} B_{1}^{n}\left(\frac{|p|}{\delta}\right) !\left(B \rho^{1 / \delta_{i}}\right)^{p+n}\left(\frac{p+n}{\delta_{i}}\right) !+\frac{F_{*}^{|p|} C_{1}^{|p|+n}\left(\frac{|p|}{\delta}\right) ! n !}{\rho^{-p-n}|p| !}\right) \\
& \left.+\sum_{p \geq 0}\left(\rho^{1 / \delta_{i}} B\right)^{p+n}\left(\frac{p+n}{\delta_{i}}\right) ! \int_{\mathcal{C}_{0}}\left|K_{p}\left(w_{0}-z_{0}\right)\right|\left|d w_{0}\right|\right) \\
& \leq A_{\rho}^{\prime} M F_{*}^{n}\left(\sum_{p \leq 0} \frac{\left(F_{*} C_{1}\right)^{|p|}\left(\frac{|p|+n}{\delta}\right) !}{\rho^{|p|}\left(\frac{|p|}{\delta_{i+1}}\right) !}\right) \\
& +A_{\rho}^{\prime} M F_{*}^{n} B_{1}^{n}\left(\frac{n}{\delta}\right) ! \sum_{-n<p<0}\left(\left(\rho^{1 / \delta_{i}} B\right)^{p+n}+C_{1}^{2|p|}\left(C_{1} \rho\right)^{p+n}\right) \\
& +A_{\rho}^{\prime} M\left(\rho^{1 / \delta_{i}} B\right)^{n}\left(A_{\theta_{0}, \rho}+\sum_{p=2}^{\infty} A_{\theta_{0}}\left(\rho^{1 / \delta_{i}} B\right)^{p} d^{\frac{p-2}{\delta_{i}}} \frac{\left(\frac{p+n}{\delta_{i}}\right) !}{\left(\frac{p}{\delta_{i}}\right) !}\right)
\end{aligned}
$$

Suppose $\gamma>\gamma_{i+1}$. Then $\delta>\delta_{i+1}$ and $\sum_{p \leq 0}\left(F_{*} C_{1}\right)^{|p|}\left(\frac{|p|+n}{\delta}\right) ! / \rho^{|p|}\left(\frac{|p|}{\delta_{i+1}}\right) !$ converges. For fixed $\rho$ choose the constant $d>0$ so small that $\sum_{p=2}^{\infty} A_{\theta_{0}}\left(\rho^{1 / \delta_{i}} B\right)^{p}$ $d^{\frac{p-2}{\delta_{i}}}\left(\frac{p+n}{\delta_{i}}\right) ! /\left(\frac{p}{\delta_{i}}\right) !$ converges. Thus $\sum_{p \in \mathbb{Z}}\left|I_{n, p}\right|$ converges in a small polydisk $U$ and there are constants $A$ and $B$ depending on $\rho$ such that $\sum_{p \in \mathbb{Z}}\left|I_{p, n}\right| \leq$ $A B^{n}\left(\frac{n}{\delta}\right)$ !. The polydisk $U$ depends on $\rho$, namely, on $\theta^{\prime}$ but not on $f(w)$. Suppose $\gamma=\gamma_{i+1}$. Then $\delta_{i+1}=\delta$. In this case first select $\rho$ with $F_{*} C_{1} \leq \rho / 2$, 
then $\sum_{p \leq 0}\left(F_{*} C_{1}\right)^{|p|}\left(\frac{|p|+n}{\delta}\right) ! / \rho^{|p|}\left(\frac{|p|}{\delta_{i+1}}\right) !$ converges. Fix $\rho$ and choose $d>0$ so small that $\sum_{p=2}^{\infty} A_{\theta_{0}}\left(\rho^{1 / \delta_{i}} B\right)^{p} d^{\frac{p-2}{\delta_{i}}}\left(\frac{p+n}{\delta_{i}}\right) ! /\left(\frac{p}{\delta_{i}}\right) !$ converges. Hence $\sum_{p \in \mathbb{Z}}\left|I_{n, p}\right|$ converges in a small polydisk $U$ and $\sum_{p \in \mathbb{Z}}\left|I_{n, p}\right| \leq A B^{n}\left(\frac{n}{\delta}\right)$ !. The polydisk $U$ depends not only on $\theta^{\prime}$ but also on $F_{*}$, which is determined by the constant $F$ appearing in the bound in $\partial_{w_{0}}^{n} f(w)$.

Convergence of $\sum_{p, q \in \mathbb{N}}\left(R_{n, p, q}^{*} f\right)(z)$. As remarked above, $\left(R_{n, p, q}^{*} f\right)(z) \equiv 0$ for $p \leq 0$, so let $p>0$. It follows from Proposition 2.6 and Lemma 4.4 that

$$
\begin{aligned}
& \left|\int_{\mathcal{C}_{0}} R_{p, n-l, 0}\left(w_{0}-z_{0}\right) \partial_{w_{0}}^{q}\left(w_{0}^{q} f\left(w_{0}, w^{\prime}\right)\right) d w_{0}\right| \\
& \leq \begin{cases}A B_{1}^{n-l} d^{\frac{p-n+l}{\delta_{i}}} \exp \left(-c\left|z_{0}\right|^{-\gamma_{i}}\right)\left(\frac{n-l-p}{\delta_{i}}\right) ! \frac{M C_{0}^{q} q !}{(\sin \eta)^{q}} & \text { for } \quad 0<p<n-l \\
\frac{A B_{1}^{n-l} d^{\frac{p-n+l}{\delta_{i}}} \exp \left(-c\left|z_{0}\right|^{-\gamma_{i}}\right)}{\left(\frac{p-n+l}{\delta_{i}}\right) !} \frac{M C_{0}^{q} q !}{(\sin \eta)^{q}} & \text { for } p \geq n-l .\end{cases}
\end{aligned}
$$

Choosing $\rho \geq 2 B C_{0} / \sin \eta$, we have by Proposition 4.2

$$
\begin{aligned}
& \sum_{p, q \in \mathbb{N}}\left|\left(R_{n, p, q}^{*} f\right)(z)\right| \leq \sum_{\substack{p, q \in \mathbb{N} \\
0 \leq l \leq n}}\left|\left(\begin{array}{l}
n \\
l
\end{array}\right) \partial_{z_{0}}^{l} k_{p, q}\left(z, w^{\prime}\right)\right| \\
& \times\left|\int_{\mathcal{C}_{0}} R_{p, n-l, 0}\left(w_{0}-z_{0}\right) \partial_{w_{0}}^{q}\left(w_{0}^{q} f\left(w_{0}, w^{\prime}\right)\right) d w_{0}\right| \leq \frac{A_{\rho} M B_{1}^{n} \exp \left(-c\left|z_{0}\right|^{-\gamma_{i}}\right)}{d^{\frac{n}{\delta_{i}}}} \\
& \times \sum_{\substack{q \in \mathbb{N} \\
0 \leq k<n}} d^{\frac{k}{\delta_{i}}} \sum_{\substack{p+l=k \\
0 \leq l \leq k}} \frac{\left(B C_{0}\right)^{q}\left(\rho^{1 / \delta_{i}} B\right)^{p}\left(\rho C / B_{1}\right)^{l}\left(\frac{p}{\delta_{i}}\right) ! n !\left(\frac{n-l-p}{\delta_{i}}\right) !}{(\rho \sin \eta)^{q}(n-l) !} \\
& +\frac{A_{\rho} M B_{1}^{n} \exp \left(-c\left|z_{0}\right|^{-\gamma_{i}}\right)}{d^{\frac{n}{\delta_{i}}}} \sum_{\substack{q \in \mathbb{N} \\
k \geq n}} d^{\frac{k}{\delta_{i}}} \sum_{\substack{p+l=k \\
0 \leq l \leq n}} \frac{\left(B C_{0}\right)^{q}\left(\rho^{1 / \delta_{i}} B\right)^{p}\left(\rho C / B_{1}\right)^{l}\left(\frac{p}{\delta_{i}}\right) ! n !}{(\rho \sin \eta)^{q}(n-l) !\left(\frac{p-n+l}{\delta_{i}}\right) !} \\
& \leq \frac{2 A_{\rho} M B_{1}^{n} \exp \left(-c\left|z_{0}\right|^{-\gamma_{i}}\right)}{d^{\frac{n}{\delta_{i}}}} \sum_{0 \leq k<n} d^{\frac{k}{\delta_{i}}} \sum_{\substack{p+l=k \\
0 \leq l \leq k}} \frac{\left(\rho^{1 / \delta_{i}} B\right)^{p}\left(\rho C / B_{1}\right)^{l}\left(\frac{p}{\delta_{i}}\right) ! n !\left(\frac{n-l-p}{\delta_{i}}\right) !}{(n-l) !} \\
& +\frac{2 A_{\rho} M B_{1}^{n} \exp \left(-c\left|z_{0}\right|^{-\gamma_{i}}\right)}{d^{\frac{n}{\delta_{i}}}} \sum_{k \geq n} \frac{d^{\frac{k}{\delta_{i}}}}{\left(\frac{k-n}{\delta_{i}}\right) !} \sum_{\substack{p+l=k \\
0 \leq l \leq n}} \frac{\left(\rho^{1 / \delta_{i}} B\right)^{p}\left(\rho C / B_{1}\right)^{l}\left(\frac{p}{\delta_{i}}\right) ! n !}{(n-l) !} \\
& \leq \frac{A_{\rho}^{\prime} M B_{1}^{n} \exp \left(-c\left|z_{0}\right|^{-\gamma_{i}}\right)}{d^{\frac{n}{\delta_{i}}}}\left(\left(\frac{n}{\delta_{i}}\right) ! \sum_{0 \leq k<n}\left((d \rho)^{1 / \delta_{i}} C_{1}\right)^{k}+\sum_{k \geq n} \frac{\left(\rho^{1 / \delta_{i}} C_{1}\right)^{k} d^{\frac{k}{\delta_{i}}}\left(\frac{k}{\delta_{i}}\right) !}{\left(\frac{k-n}{\delta_{i}}\right) !}\right) .
\end{aligned}
$$


Thus $\sum_{p, q \in \mathbb{N}}\left|\left(R_{n, p, q}^{*} f\right)(z)\right|$ converges for small $d>0$ and there are constants $A$ and $B$ depending on $\rho$ and $d$ such that

$$
\sum_{p, q \in \mathbb{N}}\left|\left(R_{n, p, q}^{*} f\right)(z)\right| \leq M A B^{n} \exp \left(-c\left|z_{0}\right|^{-\gamma_{i}}\right)\left(\frac{n}{\delta_{i}}\right) !
$$

Hence we have by $\delta \leq \delta_{i}$

$$
\left|\partial_{z_{0}}^{n}(G f)(z)\right| \leq \sum_{p \in \mathbb{Z}}\left|I_{n, p}\right|+\sum_{p, q \in \mathbb{N}}\left|\left(R_{n, p, q}^{*} f\right)(z)\right| \leq M A B^{n}\left(\frac{n}{\delta}\right) ! .
$$

The estimate of $\partial_{z_{0}}^{n}(G f)(z)$ means $(G f)(z) \in \operatorname{Asy}_{\left\{\gamma_{i}\right\}}\left(U\left(\theta^{\prime}\right)\right)$ for some $U$.

(2) Suppose $i=p^{*}-1$. Then $\delta_{i+1}=\delta_{p^{*}}=0$. In this case $\left(G_{p, q} f\right)(z) \equiv 0$ for $p<-e\left(p^{*}-1\right)$, so $(G f)(z)=\sum_{p, q \in \mathbb{N}}\left(G_{p, q} f\right)(z)$ and $\partial_{z_{0}}^{n}(G f)(z)=\sum_{p=-n}^{\infty} I_{n, p}+$ $\sum_{p, q \in \mathbb{N}}\left(R_{n, p, q}^{*} f\right)(z)$ and

$$
\begin{aligned}
I_{n, p}= & \sum_{q \in \mathbb{N}} \sum_{l=0}^{\min \{p+n, n\}}\left(\begin{array}{l}
n \\
l
\end{array}\right) \int_{\mathcal{C}^{\prime}} \partial_{z_{0}}^{l} k_{p+n-l, q}\left(z, w^{\prime}\right) d w^{\prime} \\
& \times \int_{\mathcal{C}_{0}} K_{p}\left(w_{0}-z_{0}\right) \partial_{w_{0}}^{q}\left(w_{0}^{q} f\left(w_{0}, w^{\prime}\right)\right) d w_{0} .
\end{aligned}
$$

Let $\delta>0$. Then the convergence of $\sum_{p=-n}^{\infty} I_{n, p}$ and its bound follow in the same method as above. Next let $\delta=0$. Then by (4.9) in Lemma 4.4 for $-n \leq p \leq 0$ we have

$$
\begin{aligned}
& \left|I_{n, p}\right| \leq \sum_{q=0}^{\infty}\left(\sum_{b \leq l \leq p+n} A_{\rho} B^{q}\left(\rho^{1 / \delta_{p^{*}-1} B}\right)^{p+n-l}(\rho C)^{l}\left(\frac{p+n-l}{\delta_{p^{*}-1}}\right) ! \frac{n ! M_{|p|} C_{1}^{|p|} C_{0}^{q}}{(n-l) !(\rho \sin \eta)^{q}}\right) \\
& \leq 2 A_{\rho} M_{|p|} C_{1}^{|p|} n !\left(B \rho^{1 / \delta_{p^{*}-1}}\right)^{p+n}\left(\sum_{0 \leq l<p+n} \frac{\left(\frac{C}{B}\right)^{l}\left(\frac{p+n-l}{\delta_{p^{*}-1}}\right) !}{(n-l) !}\right) \leq C_{\rho}(p, n) .
\end{aligned}
$$

For $p>0$, we have in the same way as the case $i>p^{*}-1$

$$
\begin{aligned}
\sum_{p>0}\left|I_{n, p}\right| & \leq A_{\rho}^{\prime} M_{0}\left(\sum_{p \geq 0}\left(\rho^{\left.1 / \delta_{p^{*}-1} B\right)^{p+n}}\left(\frac{p+n}{\delta_{p^{*}-1}}\right) ! \int_{\mathcal{C}_{0}} K_{p}\left(w_{0}-z_{0}\right)|| d w_{0} \mid\right)\right. \\
& \leq A_{\rho}^{\prime} M_{0}\left(\rho^{1 / \delta_{p^{*}-1}} B\right)^{n}\left(A_{\theta_{0}, \rho}+\sum_{p=2}^{\infty} A_{\theta_{0}}\left(\rho^{1 / \delta_{p^{*}-1} B}\right)^{p} d^{\frac{p-2}{\delta_{p^{*}-1}}} \frac{\left(\frac{p+n}{\delta_{i}}\right) !}{\left(\frac{p}{\delta_{p^{*}-1}}\right) !}\right)
\end{aligned}
$$


Therefore $\sum_{p=-n}^{\infty}\left|I_{n, p}\right| \leq \sum_{-n \leq p \leq 0} C_{\rho}(p, n)+\sum_{p>0}\left|I_{n, p}\right|$. By choosing small $d>0$, we have $\sum_{p>0}\left|I_{n, p}\right|<+\infty$ for $z \in U\left(\theta^{\prime}\right)$. Here $U$ is a small polydisk and does not depend on $f(w)$. The convergence and the estimate of $\sum_{p, q \in \mathbb{N}}\left(R_{p, q, n}^{*} f\right)(z)$ follow in the same way as the case $i<p^{*}-1$. Hence $(G f)(z) \in \operatorname{Asy}_{\{0\}}(U)\left(\theta^{\prime}\right)$.

(3) Further suppose $f(w) \sim 0$. Then it holds that $\lim _{z_{0} \rightarrow 0}\left(G_{n, l, p, q} f\right)(z)=$ 0 and $\lim _{z_{0} \rightarrow 0} I_{n, l}=0$. We have $(G f)(z) \sim 0$ from the above proof.

Proposition 4.6. Let $i=0$ and suppose that $f(w) \in \operatorname{Asy}_{\{\gamma\}}(W(\theta))$ with $\gamma_{1} \leq \gamma<\gamma_{0}$. Let $0<\theta^{\prime}<\theta$. Then there is a polydisk $U$ such that $(G f)(z)$ converges on $U\left(\theta^{\prime}\right)$ and $(G f)(z) \in \operatorname{Asy}_{\{\gamma\}}\left(U\left(\theta^{\prime}\right)\right)$. Moreover if $f(w) \sim 0$, then $(G f)(z) \sim 0$.

Proof. We note that $\delta_{0}=1$ and $\int_{\mathcal{C}_{0}} R_{p, n-l, 0}\left(w_{0}-z_{0}\right) \partial_{w_{0}}^{q}\left(w_{0}^{q} f\left(w_{0}, w^{\prime}\right)\right) d w_{0}$ $\equiv 0$. So $\left(R_{n, p, q}^{*} f\right)(z) \equiv 0$. The proof is almost the same as that of Proposition 4.5. The only one difference is that we use Proposition 2.7-(1) to show the convergence of $\sum_{p \in \mathbb{Z}} I_{n, p}$ (see (4.11)).

It follows from the preceding arguments that the following more precise results concerning the convergence of $(G f)(z)$ hold.

Theorem 4.7. Suppose that $p^{*} \geq 2, i \in\left\{1,2, \ldots, p^{*}-1\right\}$, Condition $\left(\mathrm{C}_{i}\right)$ holds and $f(z) \in \operatorname{Asy}_{\{\gamma\}}(W(\theta))$ with $\gamma_{i+1} \leq \gamma<\gamma_{i}$. Let $0<$ $\theta^{\prime}<\min \left\{\frac{\pi}{2 \gamma_{i}}, \theta\right\}$. Then there is a polydisk $U \subset W$ such that $(G f)(z)=$ $\sum_{p, q \in \mathbb{Z}}\left(G_{p, q} f\right)(z)$ absolutely converges on $U\left(\theta^{\prime}\right)$ and $(G f)(z) \in \operatorname{Asy}_{\{\gamma\}}\left(U\left(\theta^{\prime}\right)\right)$. The dependence of $U$ is the following.

(1) If $i \neq p^{*}-1$ and $\gamma>\gamma_{i+1}$, then $U$ depends on $\theta^{\prime}$ but not on $f(z)$.

(2) If $i \neq p^{*}-1$ and $\gamma=\gamma_{i+1}$, then $U$ depends both $\theta^{\prime}$ and $f(z)$.

(3) If $i=p^{*}-1$, then $U$ depend on $\theta^{\prime}$ but not on $f(z)$.

Theorem 4.8. Suppose that Condition $\left(\mathrm{C}_{0}\right)$ holds and $f(z) \in \operatorname{Asy}_{\{\gamma\}}$ $(W(\theta))$ with $\gamma \geq \gamma_{1}$. Let $0<\theta^{\prime}<\theta$. Then there is a polydisk $U$ such that $(G f)(z)=\sum_{p, q \in \mathbb{Z}}\left(G_{p, q} f\right)(z)$ absolutely converges on $U\left(\theta^{\prime}\right)$ and $(G f)(z) \in$ $\operatorname{Asy}_{\{\gamma\}}\left(U\left(\theta^{\prime}\right)\right)$. The dependence of $U$ is the following.

(1) If $p^{*}=1$ or $\gamma>\gamma_{1}$, then $U$ depends on $\theta^{\prime}$ but not on $f(z)$.

(2) If $p^{*} \geq 2$ and $\gamma=\gamma_{1}$, then $U$ depends both $\theta^{\prime}$ and $f(z)$. 
Under the assumption that for fixes $i$ Condition $\left(\mathrm{C}_{i}\right)$ holds, we have defined $(G f)(z)$ for $f(z) \in \operatorname{Asy}_{\{\gamma\}}(W(\theta))$ and shown $(G f)(z) \in \operatorname{Asy}_{\{\gamma\}}\left(U\left(\theta^{\prime}\right)\right)$. Let us write again the form of $(G f)(z)$,

$$
\begin{aligned}
(G f)(z) & =\int_{\mathcal{C}} G\left(w_{0}-z_{0}, z, w\right) f(w) d w \\
& =\sum_{p=-\infty}^{+\infty} \sum_{q=0}^{+\infty} \int_{\mathcal{C}} k_{p, q}\left(z, w^{\prime}\right) K_{p, q}\left(w_{0}-z_{0}, w_{0}\right) f(w) d w
\end{aligned}
$$

We show that $g(z):=(G f)(z)$ and $(R f)(z):=P\left(z, \partial_{z}\right)(G f)(z)-f(z)$ have desired properties in Theorem 1.2. For this purpose we calculate $P\left(z, \partial_{z}\right)(G f)(z)$.

Lemma 4.9. $(R f)(z)$ is written as follows:

$$
\begin{aligned}
(R f)(z)= & \sum_{\alpha, p, q}\left(R_{\alpha, p, q}^{\#} f\right)(z) \\
\left(R_{\alpha, p, q}^{\#} f\right)(z)= & \sum_{\substack{0 \leq l \leq \alpha_{0} \\
s+s^{\prime} \leq j_{\alpha}}} C_{j_{\alpha}, q^{\prime}+\alpha_{0}-l, s, s^{\prime}} b_{\alpha, l}(z) \int_{\mathcal{C}^{\prime}} \partial_{z^{\prime}}^{\alpha^{\prime}} \partial_{z_{0}}^{l} k_{p, q}\left(z, w^{\prime}\right) d w^{\prime} \\
& \times \int_{\mathcal{C}_{0}} R_{p,-e_{\alpha}-l, s}\left(w_{0}-z_{0}\right) \partial_{w_{0}}^{q+s^{\prime}}\left(w_{0}^{q+s^{\prime}} f\left(w_{0}, w^{\prime}\right)\right) d w_{0}
\end{aligned}
$$

where $b_{\alpha, l}(z)=\left(\begin{array}{c}\alpha_{0} \\ l\end{array}\right) b_{\alpha}(z)$.

Proof. By Proposition 3.4 we have

$$
\begin{aligned}
& z_{0}^{j_{\alpha}} b_{\alpha}(z) \partial_{z}^{\alpha} \int_{\mathcal{C}^{\prime}} k_{p, q}\left(z, w^{\prime}\right) d w^{\prime} \int_{\mathcal{C}_{0}} K_{p, q}\left(w_{0}-z_{0}, w_{0}\right) f\left(w_{0}, w^{\prime}\right) d w_{0} \\
& =\sum_{l=0}^{\alpha_{0}} b_{\alpha, l}(z) \int_{\mathcal{C}^{\prime}} \partial_{z^{\prime}}^{\alpha^{\prime}} \partial_{z_{0}}^{l} k_{p, q}\left(z, w^{\prime}\right) d w^{\prime}\left(\sum_{s+s^{\prime} \leq j_{\alpha}} C_{j_{\alpha}, q+\alpha_{0}-l, s, s^{\prime}}\right. \\
& \left.\quad \times \int_{\mathcal{C}_{0}} p^{s} K_{p+e_{\alpha}+l, q+s^{\prime}}\left(w_{0}-z_{0}, w_{0}\right) f\left(w_{0}, w^{\prime}\right) d w_{0}\right)+\left(R_{\alpha, p, q}^{\#} f\right)(z) .
\end{aligned}
$$


$\left(R_{\alpha, p, q}^{\#} f\right)(z)$ is given by (3.10) in Proposition 3.4. We have by (3.26) and (3.15)

$$
\begin{aligned}
& P\left(z, \partial_{z}\right)(G f)(z)=\sum_{p, q, \alpha} \sum_{l=0}^{\alpha_{0}} b_{\alpha, l}(z)\left(\sum_{s+s^{\prime} \leq j_{\alpha}} C_{j_{\alpha}, q+\alpha_{0}-l, s, s^{\prime}}\right. \\
& \left.\times \int_{\mathcal{C}} \partial_{z^{\prime}}^{\alpha^{\prime}} \partial_{z_{0}}^{l} k_{p, q}\left(z, w^{\prime}\right) p^{s} K_{p+e_{\alpha}+l, q+s^{\prime}}\left(z_{0}-w_{0}, w_{0}\right) f(w) d w\right)+(R f)(z)
\end{aligned}
$$

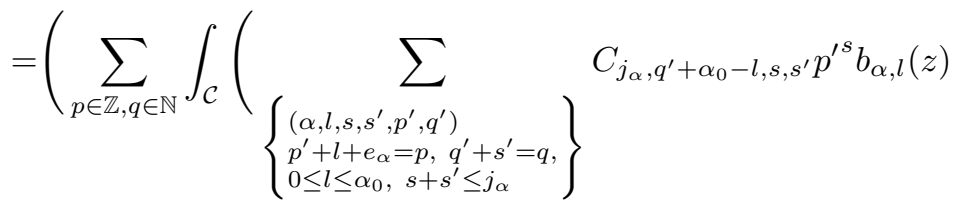

$$
\begin{aligned}
& \left.\left.\times \partial_{z^{\prime}}^{\alpha^{\prime}} \partial_{z_{0}}^{l} k_{p^{\prime}, q^{\prime}}\left(z, w^{\prime}\right)\right) K_{p, q}\left(z_{0}-w_{0}, w_{0}\right) f(w) d w\right)+(R f)(z) \\
& =\frac{1}{(2 \pi i)^{d}} \int_{\mathcal{C}} \frac{K_{0,0}\left(z_{0}-w_{0}, w_{0}\right)}{\prod_{i=1}^{d}\left(w_{i}-z_{i}\right)} f(w) d w+R f(z)=f(z)+(R f)(z),
\end{aligned}
$$

where $(R f)(z)=\sum_{\alpha, p, q}\left(R_{\alpha, p, q}^{\#} f\right)(z)$.

The next purpose is to estimate $(R f)(z)$.

Proposition 4.10. Suppose that condition $\left(\mathrm{C}_{i}\right)$ holds for fixed $i \neq 0$. Let $f(z) \in \mathcal{O}(W(\theta))$ with $0<\theta<\pi / 2 \gamma_{i}$ and $0<\theta^{\prime}<\theta_{0}<\theta$. Put $M=$ $\sup _{z \in W\left(\theta_{0}\right)}|f(z)|$. Then there are positive constants $A$ and $c$ and a polydisk $U$ depending on $\theta^{\prime}$ such that $(R f)(z)$ converges on $U\left(\theta^{\prime}\right)$ and

$$
|(R f)(z)| \leq A M \exp \left(-c\left|z_{0}\right|^{-\gamma_{i}}\right) .
$$

Proof. Define $h, s_{0} \in \mathbb{N}$ as follows:

$$
h=\max _{\alpha} \max _{0 \leq l \leq \alpha_{0}}\left|e_{\alpha}+l\right|, \quad s_{0}=\max _{\alpha} j_{\alpha},
$$

where $\alpha \in \mathbb{N}^{d+1}$ with $a_{\alpha}(z) \not \equiv 0$. Let $V \Subset W$ be a polydisk centered at the origin and $0<\eta<\theta_{0}-\theta^{\prime}$. Then by Lemma $4.4 \mid \partial_{w_{0}}^{q}\left(w_{0}^{q} f\left(w_{0}, w^{\prime}\right) \mid \leq M C_{0}^{q} q ! /(\sin \eta)^{q}\right.$ for $z \in V\left(\theta_{0}-\eta\right)$. By Proposition 2.6

$$
\int_{\mathcal{C}_{0}} R_{p,-e_{\alpha}-l, s}\left(w_{0}-z_{0}\right) \partial_{w_{0}}^{q+s^{\prime}}\left(w_{0}^{q+s^{\prime}} f\left(w_{0}, w^{\prime}\right)\right) d w_{0} \in \mathcal{O}\left(V\left(\theta^{\prime}\right)\right)
$$


and if $p \leq-h$, it is identically zero. Hence $\left(R_{\alpha, p, q}^{\#} f\right)(z)=0$ for $p \leq-h$. Suppose $p \geq h$. Then

$$
\begin{aligned}
& \left|\int_{\mathcal{C}_{0}} R_{p,-e_{\alpha}-l, s}\left(w_{0}-z_{0}\right) \partial_{w_{0}}^{q+s^{\prime}}\left(w_{0}^{q+s^{\prime}} f\left(w_{0}, w^{\prime}\right)\right) d w_{0}\right| \\
& \leq \frac{A B^{h}(1+|p|)^{s_{0}} d^{\frac{p-h}{\delta_{i}}} e^{-c\left|z_{0}\right|^{-\gamma_{i}}} M C_{0}^{q+s^{\prime}}\left(q+s^{\prime}\right) !}{\left(\frac{p-h}{\delta_{i}}\right) !(\sin \eta)^{q+s^{\prime}}} .
\end{aligned}
$$

By Proposition 4.2 we have

$$
\begin{aligned}
& \left|\left(R_{\alpha, p, q}^{\#} f\right)(z)\right| \leq \sum_{\substack{0 \leq l \leq \alpha_{0} \\
s+s^{\prime} \leq j_{\alpha}}}\left|C_{j_{\alpha}, q+\alpha_{0}-l, s, s^{\prime}} b_{\alpha}(z)\right| \int_{\mathcal{C}^{\prime}}\left|\partial_{z^{\prime}}^{\alpha^{\prime}} \partial_{z_{0}}^{l} k_{p, q}\left(z, w^{\prime}\right)\right|\left|d w^{\prime}\right| \\
& \times\left|\int_{\mathcal{C}_{0}} R_{p,-e_{\alpha}-l, s}\left(w_{0}-z_{0}\right) \partial_{w_{0}}^{q+s^{\prime}}\left(w_{0}^{q+s^{\prime}} f\left(w_{0}, w^{\prime}\right)\right) d w_{0}\right| \\
& \leq \frac{A^{\prime} B^{h} M(1+|p|)^{s_{0}} d^{\frac{p-h}{\delta_{i}}} e^{-c\left|z_{0}\right|^{-\gamma_{i}}}}{\left(\frac{p-h}{\delta_{i}}\right) !} \int_{\mathcal{C}^{\prime}}\left|\partial_{z^{\prime}}^{\alpha^{\prime}} \partial_{z_{0}}^{l} k_{p, q}\left(z, w^{\prime}\right)\right|\left|d w^{\prime}\right| \\
& \times\left(\sum_{s+s^{\prime} \leq j_{\alpha}} \frac{C_{0}^{q+s^{\prime}}\left(q+s^{\prime}\right) !}{(\sin \eta)^{q+s^{\prime}}}\left|C_{j_{\alpha}, q+\alpha_{0}-l, s, s^{\prime}}\right|\right) \\
& \leq \frac{A_{\rho}^{\prime} M(1+|p|)^{s_{0}} d^{\frac{p-h}{\delta_{i}}} e^{-c\left|z_{0}\right|^{-\gamma}}}{\left(\frac{p-h}{\delta_{i}}\right) !} \frac{B^{q}\left(\rho^{1 / \delta_{i}} B\right)^{p}\left(\frac{p}{\delta_{i}}\right) !}{\rho^{q} q !} \\
& \times\left(\sum_{s+s^{\prime} \leq j_{\alpha}} \frac{C_{0}^{q+s^{\prime}}\left(q+s^{\prime}\right) !(1+q)^{j_{\alpha}-s-s^{\prime}}}{(\sin \eta)^{q+s^{\prime}}}\right) \\
& \leq A_{\rho}^{\prime \prime} M e^{-c\left|z_{0}\right|^{-\gamma_{i}}}(1+|p|)^{s_{0}+\frac{h}{\delta_{i}}} d^{\frac{p-h}{\delta_{i}}}\left(\rho^{1 / \delta_{i}} B\right)^{p} \frac{B^{q} C_{0}^{q+j_{\alpha}}\left(q+j_{\alpha}\right) !}{\rho^{q} q !(\sin \eta)^{q+j_{\alpha}}} .
\end{aligned}
$$

Put $\left(R_{\alpha, p}^{\#} f\right)(z)=\sum_{q \in \mathbb{N}}\left(R_{\alpha, p, q}^{\#} f\right)(z)$. Select $\rho$ so that $\rho \geq 2 B C_{0} / \sin \eta$. Then $\left(R_{\alpha, p}^{\#} f\right)(z)$ converges and

$$
\left|\left(R_{\alpha, p}^{\#} f\right)(z)\right| \leq A_{\rho} M e^{-c\left|z_{0}\right|^{-\gamma_{i}}}(1+|p|)^{s_{0}+\frac{h}{\delta_{i}}} d^{\frac{p-h}{\delta_{i}}}\left(\rho^{1 / \delta_{i}} B\right)^{p}
$$

where $\rho$ depends on $\eta$ but not on $p$. If $|p|<h$, by Proposition 2.6 we have in 
the same way as above

$$
\begin{aligned}
& \left|\int_{\mathcal{C}_{0}} R_{p,-e_{\alpha}-l, s}\left(w_{0}-z_{0}\right) \partial_{w_{0}}^{q+s^{\prime}}\left(w_{0}^{q+s^{\prime}} f\left(w_{0}, w^{\prime}\right)\right) d w_{0}\right| \\
& \leq \frac{A_{\rho} B^{h}(1+|p|)^{s_{0}} d^{\frac{p-h}{\delta_{i}}} \exp \left(-c\left|z_{0}\right|^{-\gamma_{i}}\right) M C_{0}^{q+s^{\prime}}\left(q+s^{\prime}\right) !}{\rho^{q} q !(\sin \eta)^{q+s^{\prime}}} \\
& \quad \leq \frac{A_{\rho}^{\prime} d^{\frac{p-h}{\delta_{i}}} \exp \left(-c\left|z_{0}\right|^{-\gamma_{i}}\right) M C_{0}^{q+s^{\prime}}\left(q+s^{\prime}\right) !}{\rho^{q} q !(\sin \eta)^{q+s^{\prime}}}
\end{aligned}
$$

Hence if $\rho \geq 2 C_{0} / \sin \eta$, then $\left(R_{\alpha, p}^{\#} f\right)(z)$ converges and

$$
\left|\left(R_{\alpha, p}^{\#} f\right)(z)\right| \leq A_{\rho} M \exp \left(-c\left|z_{0}\right|^{-\gamma_{i}}\right) d^{\frac{p-h}{\delta_{i}}}\left(\rho^{1 / \delta_{i}} B\right)^{p}
$$

holds. Choose $d>0$ so small for fixed $\rho$ such that $d \rho^{1 / \delta_{i}} B \leq 1 / 2$, then $(R f)(z)=\sum_{\alpha, p>-h}\left(R_{\alpha, p}^{\#} f\right)(z)$ converges and (4.16) holds.

We remark that the parameter $d$ contained in the operator $(G f)(z)$ and $(R f)(z)$ is chosen so small and fixed. Originally $d$ is the parameter appearing in the definition of $\hat{g}_{p}(\lambda)$. Therefore the functions derived from $\hat{g}_{p}(\lambda)$, for example, $K_{p, q}\left(w_{0}-z_{0}, w_{0}\right)$, depend on $d$.

Thus if $i \neq 0$, we have from Theorem 4.7 and Proposition 4.10.

Theorem 4.11. Suppose that conditions in Theorem 4.7 hold. Then there is a polydisk $U$ such that $(G f)(z) \in \operatorname{Asy}_{\{\gamma\}}\left(U\left(\theta^{\prime}\right)\right)$ and $(R f)(z) \sim 0$ in $A s y_{\left\{\gamma_{i}\right\}}\left(U\left(\theta^{\prime}\right)\right)$.

We have for $i=0$.

Theorem 4.12. Suppose that conditions in Theorem 4.8 hold. Then there is a polydisk $U$ such that $(G f)(z) \in \operatorname{Asy}_{\{\gamma\}}\left(U\left(\theta^{\prime}\right)\right)$ and $P\left(z, \partial_{z}\right)(G f)(z)=$ $f(z)$.

Proof. For $i=0$, we have $\delta_{0}=1$. It follows from Proposition 2.6 that $\int_{\mathcal{C}_{0}} R_{p,-e_{\alpha}-l, s}\left(w_{0}-z_{0}\right) \partial_{w_{0}}^{q+s^{\prime}}\left(w_{0}^{q+s^{\prime}} f\left(w_{0}, w^{\prime}\right)\right) d w_{0}=0$, hence $(R f)(z)=0$.

Thus from Theorems in this section we conclude that $g(z)=(G f)(z)$ has desired properties in Theorem 1.2. Theorem 1.3 follows from Theorem 1.2.

Proof of Theorem 1.3. We assume conditions $\left(\mathrm{C}_{i}\right)$ for $i=0,1, \ldots, s$. Let $f(z) \in \operatorname{Asy}_{\{\gamma\}}(\Omega)(\theta)$ with $\gamma \geq \gamma_{s+1}$. Let $\theta^{\prime}=\theta_{0}<\theta_{1}<\cdots<\theta_{s}$ be constants 
with $\theta_{i}<\min \left\{\theta, \pi / 2 \gamma_{i}\right\}$ for $i \geq 1$. It follows from condition $\left(\mathrm{C}_{s}\right)$ and Theorem 1.2 that there is a $g_{s}(z) \in \operatorname{Asy}_{\{\gamma\}}\left(U_{s}\left(\theta_{s}\right)\right)$ for some polydisk $U_{s}$ such that

$$
P\left(z, \partial_{z}\right) g_{s}(z)=f(z)+r_{s}(z),
$$

where $r_{s}(z) \sim 0$ in $\operatorname{Asy}_{\left\{\gamma_{s}\right\}}\left(U_{s}\left(\theta_{s}\right)\right)$. By condition $\left(C_{s-1}\right)$ there is a $g_{s-1}(z) \in$ $\operatorname{Asy}_{\left\{\gamma_{s}\right\}}\left(U_{s-1}\left(\theta_{s-1}\right)\right)$ for some polydisk $U_{s-1}$ such that

$$
P\left(z, \partial_{z}\right) g_{s-1}(z)=-r_{s}(z)+r_{s-1}(z),
$$

where $r_{s-1}(z) \sim 0$ in $\operatorname{Asy}_{\left\{\gamma_{s-1}\right\}}\left(U_{s-1}\left(\theta_{s-1}\right)\right)$. By repeating the argument, from condition $\left(C_{i}\right)$ there is a $g_{i}(z) \in \operatorname{Asy}_{\left\{\gamma_{i+1}\right\}}\left(U_{i}\left(\theta_{i}\right)\right)$ for some polydisk $U_{i}$ such that

$$
P\left(z, \partial_{z}\right) g_{i}(z)=-r_{i+1}(z)+r_{i}(z)
$$

where $r_{i}(z) \sim 0$ in $\operatorname{Asy}_{\left\{\gamma_{i}\right\}}\left(U_{i}\left(\theta_{i}\right)\right)$. Finally it follows from condition $\left(C_{0}\right)$ that there is an exact solution $g_{0}(z) \in \operatorname{Asy}_{\left\{\gamma_{1}\right\}}\left(U_{0}\left(\theta_{0}\right)\right)$ of

$$
P\left(z, \partial_{z}\right) g_{0}(z)=-r_{1}(z)
$$

for some polydisk $U_{0}$. Consequently $u(z)=\sum_{i=0}^{s} g_{i}(z) \in \operatorname{Asy}_{\{\gamma\}}\left(U\left(\theta^{\prime}\right)\right), U:=$ $U_{0}$, satisfies $P\left(z, \partial_{z}\right) u(z)=f(z)$.

\section{$\S 5 . \quad$ Proof of Proposition 2.3}

In this section we give the proof of Proposition 2.3 and $d$ is a positive constant. For this purpose we give lemmas.

Lemma 5.1. Let $a, d$ and $s$ be positive constants. Then for $0<\eta<1$

$$
\int_{d}^{\infty} e^{-a x} x^{s} d x \leq \frac{e^{-\eta a d} s !}{((1-\eta) a)^{s+1}} .
$$

Proof. We have

$$
\int_{d}^{\infty} e^{-a x} x^{s} d x \leq e^{-\eta a d} \int_{d}^{\infty} e^{-(1-\eta) a x} x^{s} d x \leq \frac{e^{-\eta a d} s !}{((1-\eta) a)^{s+1}} .
$$

Lemma 5.2. Let $c, \delta$ and $h$ be positive constants. Then

$$
\sup _{t \geq 0} e^{-c t^{\delta}} t^{h} \leq A c^{-\frac{h}{\delta}}\left(\frac{h}{\delta}\right) ! .
$$

where $A$ is independent of $c, \delta$ and $h$. 
Proof. We have by Stirling's formula

$$
\sup _{t \geq 0} e^{-c t^{\delta}} t^{h}=c^{-\frac{h}{\delta}} \sup _{x \geq 0} e^{-x} x^{\frac{h}{\delta}}=c^{-\frac{h}{\delta}}\left(\frac{h}{\delta}\right)^{\frac{h}{\delta}} e^{-\frac{h}{\delta}} \leq A c^{-\frac{h}{\delta}}\left(\frac{h}{\delta}\right) ! .
$$

Proposition 2.3 gives the estimate of $\hat{r}_{p, h, s}(\lambda)=p^{s} \hat{r}_{p, h}^{0}(\lambda)+\lambda^{h} \hat{r}_{p, s}^{1}(\lambda)$, where $\hat{r}_{p, h}^{0}(\lambda):=\lambda^{h} \hat{g}_{p}(\lambda)-\hat{g}_{p-h}(\lambda)$ (see $\left.(2.8)\right)$. As for the estimate of $\hat{r}_{p, s}^{0}(\lambda)$ we have

Lemma 5.3. (1) Let $h \geq 0$.

(1-i) If $p \leq 0$, then $\hat{r}_{p, h}^{0}(\lambda)=0$.

(1-ii) Suppose $|\arg \lambda|<\phi<\frac{\pi}{2 \delta}$. If $0<p \leq h$, then there are constants $A=A(\phi)$ and $B=B(\phi)$ such that

$$
\left|\hat{r}_{p, h}^{0}(\lambda)\right| \leq A B^{h} d^{\frac{p-h}{\delta}} e^{-\frac{d(\cos \delta \phi)}{2}|\lambda|^{\delta}}\left(\frac{h-p}{\delta}\right) ! .
$$

If $p>h$, then there are constants $A=A(\phi)$ and $B=B(\phi)$ such that

$$
\left|\hat{r}_{p, h}^{0}(\lambda)\right| \leq \frac{A B^{h} d^{\frac{p-h}{\delta}} e^{-\frac{d(\cos \delta \phi)}{2}|\lambda|^{\delta}}}{\left(\frac{p-h}{\delta}\right) !} .
$$

(2) Let $h \leq 0$. Then $\hat{r}_{p, h}^{0}(\lambda)=-\lambda^{h} \hat{r}_{p-h,-h}^{0}(\lambda)$.

(2-i) If $p \leq h$, then $\hat{r}_{p, h}^{0}(\lambda)=0$.

(2-ii) Suppose $|\arg \lambda|<\phi<\frac{\pi}{2 \delta}$. If $h<p \leq 0$, then there are constants $A=A(\phi)$ and $B=B(\phi)$ such that

$$
\left|\hat{r}_{p, h}^{0}(\lambda)\right| \leq A B^{|h|} d^{\frac{p}{\delta}}|\lambda|^{-|h|} e^{-\frac{d(\cos \delta \phi)}{2}|\lambda|^{\delta}}\left(\frac{|p|}{\delta}\right) ! .
$$

If $p>0$, then there are constants $A=A(\phi)$ and $B=B(\phi)$ such that

$$
\left|\hat{r}_{p, h}^{0}(\lambda)\right| \leq \frac{A B^{|h|} d^{\frac{p}{\delta}} e^{-\frac{d(\cos \delta \phi)}{2}|\lambda|^{\delta}}}{|\lambda|^{|h|}\left(\frac{p}{\delta}\right) !} .
$$

Proof. (1). Let $h \geq 0$. If $p \leq 0, \lambda^{h} \hat{g}_{p}(\lambda)=\lambda^{h-p}=\hat{g}_{p-h}(\lambda)$. Hence $\hat{r}_{p, h}^{0}(\lambda)=0$. Suppose $0<p \leq h$. Then we have

$$
\lambda^{h} \hat{g}_{p}(\lambda)=\frac{\lambda^{h+\delta}}{\left(\frac{p}{\delta}\right) !} \int_{0}^{d} e^{-\lambda^{\delta} \zeta} \zeta^{\frac{p}{\delta}} d \zeta=\lambda^{h-p}+\hat{r}_{p, h}^{0}(\lambda)=\hat{g}_{p-h}(\lambda)+\hat{r}_{p, h}^{0}(\lambda)
$$


with

$$
\hat{r}_{p, h}^{0}(\lambda)=-\frac{\lambda^{h+\delta}}{\left(\frac{p}{\delta}\right) !} \int_{d}^{\infty} e^{-\lambda^{\delta} \zeta} \zeta^{\frac{p}{\delta}} d \zeta .
$$

By Lemmas 5.1 and 5.2 there are constants $A=A(\phi)$ and $B=B(\phi)$ such that for $|\arg \lambda|<\phi<\frac{\pi}{2 \delta}$

$$
\begin{aligned}
\left|\hat{r}_{p, h}^{0}(\lambda)\right| & \leq|\lambda|^{h-p} e^{-\frac{3}{4} d \cos (\delta \phi)|\lambda|^{\delta}}\left(\frac{4}{\cos \delta \phi}\right)^{\frac{p}{\delta}+1} \\
& =\left(\frac{4}{\cos \delta \phi}\right)^{\frac{p}{\delta}+1} e^{-\frac{1}{2} d \cos (\delta \phi)} \times e^{-\frac{1}{4} d \cos (\delta \phi)|\lambda|^{\delta}}|\lambda|^{h-p} \\
& \leq A^{\prime}\left(\frac{4}{\cos \delta \phi}\right)^{\frac{p}{\delta}+1} e^{-\frac{1}{2} d \cos (\delta \phi)}\left(\frac{4}{d \cos \delta \phi}\right)^{\frac{h-p}{\delta}}\left(\frac{h-p}{\delta}\right) ! \\
& \leq A B^{h} d^{\frac{p-h}{\delta}} e^{-\frac{1}{2} d \cos (\delta \phi)}\left(\frac{h-p}{\delta}\right) !
\end{aligned}
$$

This implies (5.4). Next suppose $p>h$. Then we have

$$
\begin{aligned}
\lambda^{-h} \hat{g}_{p-h}(\lambda) & =\frac{\lambda^{2 \delta}}{\left(\frac{h}{\delta}\right) !\left(\frac{p-h}{\delta}\right) !} \int_{0}^{\infty} e^{-\lambda^{\delta} \zeta} \zeta^{\frac{h}{\delta}} d \zeta \int_{0}^{d} e^{-\lambda^{\delta} \eta} \eta^{\frac{p-h}{\delta}} d \eta \\
& =\frac{\lambda^{2 \delta}}{\left(\frac{h}{\delta}\right) !\left(\frac{p-h}{\delta}\right) !} \int_{0}^{d} e^{-\lambda^{\delta} x} d x \int_{0}^{x}(x-y)^{\frac{h}{\delta}} y^{\frac{p-h}{\delta}} d y \\
& +\frac{\lambda^{2 \delta}}{\left(\frac{h}{\delta}\right) !\left(\frac{p-h}{\delta}\right) !} \int_{d}^{\infty} e^{-\lambda^{\delta} x} d x \int_{0}^{d}(x-y)^{\frac{h}{\delta}} y^{\frac{p-h}{\delta}} d y=\mathrm{I}+\mathrm{II} .
\end{aligned}
$$

By using the relation of Gamma function and Beta function, we have

$$
\begin{aligned}
\mathrm{I} & =\frac{\lambda^{2 \delta}}{\left(\frac{p}{\delta}+1\right) !} \int_{0}^{d} e^{-\lambda^{\delta} x} x^{\frac{p}{\delta}+1} d x=\frac{\lambda^{\delta}}{\left(\frac{p}{\delta}\right) !} \int_{0}^{d} e^{-\lambda^{\delta} x} x^{\frac{p}{\delta}} d x-\frac{\lambda^{\delta} e^{-\lambda^{\delta} d} d^{\frac{p}{\delta}+1}}{\left(\frac{p}{\delta}+1\right) !} \\
& =\hat{g}_{p}(\lambda)-\frac{\lambda^{\delta} e^{-\lambda^{\delta} d} d^{\frac{p}{\delta}+1}}{\left(\frac{p}{\delta}+1\right) !}
\end{aligned}
$$

Hence

$$
\hat{r}_{p, h}^{0}(\lambda)=-\lambda^{h} \mathrm{II}+\frac{\lambda^{\delta+h} e^{-\lambda^{\delta} d} d^{\frac{p}{\delta}+1}}{\left(\frac{p}{\delta}+1\right) !} .
$$


As for $\lambda^{h}$ II we have

$$
\begin{aligned}
\left|\lambda^{h} \mathrm{II}\right| & \leq \frac{|\lambda|^{2 \delta+h} d^{\frac{p-h}{\delta}+1}}{\left(\frac{h}{\delta}\right) !\left(\frac{p-h}{\delta}\right) !\left(\frac{p-h}{\delta}+1\right)}\left|\int_{d}^{\infty} e^{-\lambda^{\delta} x} x^{\frac{h}{\delta}} d x\right| \\
& \leq \frac{|\lambda|^{\delta} d^{\frac{p-h}{\delta}+1} e^{-\frac{3 d \cos (\delta \phi)}{4}|\lambda|^{\delta}}}{\left(\frac{p-h}{\delta}+1\right) !\left(\frac{\cos \delta \phi}{4}\right)^{\frac{h}{\delta}+1}} \leq A_{1} B_{1}^{h} \frac{d^{\frac{p-h}{\delta}} e^{-\frac{d \cos (\delta \phi)}{2}|\lambda|^{\delta}}}{\left(\frac{p-h}{\delta}+1\right) !} .
\end{aligned}
$$

Therefore

$$
\begin{aligned}
\left|\hat{r}_{p, h}^{0}(\lambda)\right| & \leq A_{1} B_{1}^{h} \frac{d^{\frac{p-h}{\delta}} e^{-\frac{d \cos (\delta \phi)}{2}|\lambda|^{\delta}}}{\left(\frac{p-h}{\delta}+1\right) !}+\frac{d^{\frac{p}{\delta}} e^{-\frac{d \cos (\delta \phi)}{2}|\lambda|^{\delta}}}{\left(\frac{p}{\delta}+1\right) !} \times e^{-\frac{d \cos (\delta \phi)}{2}|\lambda|^{\delta}} d|\lambda|^{\delta+h} \\
& \leq A_{1} B_{1}^{h} \frac{d^{\frac{p-h}{\delta}} e^{-\frac{d \cos (\delta \phi)}{2}|\lambda|^{\delta}}}{\left(\frac{p-h}{\delta}+1\right) !}+A_{2} B_{2}^{h} \frac{d^{\frac{p-h}{\delta}} e^{-\frac{d \cos (\delta \phi)}{2}|\lambda|^{\delta}}\left(\frac{h}{\delta}\right) !}{\left(\frac{p}{\delta}+1\right) !} \\
& \leq A B^{h} \frac{d^{\frac{p-h}{\delta}} e^{-\frac{d \cos (\delta \phi)}{2}|\lambda|^{\delta}}}{\left(\frac{p-h}{\delta}\right) !}
\end{aligned}
$$

where constants $A_{1}, B_{1}, A_{2}, B_{2}, A$ and $B$ depend only on $\phi$. Thus we have (5.5). By the definition of $\hat{r}_{p, h}^{0}(\lambda)$ we have $\hat{r}_{p, h}^{0}(\lambda)=-\lambda^{h} \hat{r}_{p-h,-h}^{0}(\lambda)$. Hence the estimates of $\hat{r}_{p, h}^{0}(\lambda)$ for $h<0$ in (2) follow from those for $h \geq 0$ in (1).

Proof of Proposition 2.3. We have to estimate $\hat{r}_{p, s}^{1}(\lambda)$. Since $0 \leq s \leq s_{0}$, it follows from $(2.8)$ that $\left|\hat{r}_{p, s}^{1}(\lambda)\right| \leq A d^{\frac{p}{\delta}}(1+p)^{s_{0}-1} e^{-\frac{3 d \cos \phi_{0}}{4}} /\left(\frac{p}{\delta}\right)$ ! for $p>0$ and $\hat{r}_{p, s}^{1}(\lambda)=0$ for $p \leq 0$. Now suppose $h \geq 0$. If $p \leq 0$, it is obvious that $\hat{r}_{p, h, s}(\lambda)=0$. If $0<p<h$, then

$$
\begin{aligned}
& \left|\hat{r}_{p, h, s}(\lambda)\right| \leq p^{s}\left|\hat{r}_{p, h}^{0}(\lambda)\right|+|\lambda|^{h}\left|\hat{r}_{p, s}^{1}(\lambda)\right| \\
\leq & p^{s} A B^{h} d^{\frac{p-h}{\delta}} e^{-\frac{d(\cos \phi)}{2}|\lambda|^{\delta}}\left(\frac{h-p}{\delta}\right) ! \\
& +\frac{A d^{\frac{p-h}{\delta}}(1+p)^{s_{0}-1} e^{-\frac{d \cos \phi_{0}}{2}|\lambda|^{\delta}} e^{-\frac{d \cos \phi_{0}}{4}|\lambda|^{\delta}}\left(|\lambda|^{\delta} d\right)^{\frac{h}{\delta}}}{\left(\frac{p}{\delta}\right) !} \\
\leq & A_{1}(1+p)^{s_{0}} B_{1}^{h} d^{\frac{p-h}{\delta}} e^{-\frac{d(\cos \phi)}{2}|\lambda|^{\delta}}\left(\frac{h-p}{\delta}\right) ! \quad \text { (by Lemma 5.2). }
\end{aligned}
$$


If $p \geq h$, then

$$
\begin{aligned}
& \left|\hat{r}_{p, h, s}(\lambda)\right| \leq p^{s}\left|\hat{r}_{p, h}^{0}(\lambda)\right|+|\lambda|^{h}\left|\hat{r}_{p, s}^{1}(\lambda)\right| \\
\leq & \frac{p^{s} A B^{h} d^{\frac{p-h}{\delta}} e^{-\frac{d(\cos \phi)}{2}|\lambda|^{\delta}}}{\left(\frac{p-h}{\delta}\right) !}+\frac{|\lambda|^{h} A d^{\frac{p}{\delta}}(1+p)^{s_{0}-1} e^{-\frac{3 d \cos \phi_{0}}{4}|\lambda|^{\delta}}}{\left(\frac{p}{\delta}\right) !} \\
\leq & \frac{A(1+p)^{s_{0}} B^{h} d^{\frac{p-h}{\delta}} e^{-\frac{d(\cos \phi)}{2}|\lambda|^{\delta}}}{\left(\frac{p-h}{\delta}\right) !} \\
+ & \frac{A d^{\frac{p-h}{\delta}}(1+p)^{s_{0}-1} e^{-\frac{d \cos \phi_{0}}{2}|\lambda|^{\delta}} e^{-\frac{d \cos \phi_{0}}{4}|\lambda|^{\delta}\left(|\lambda|^{\delta} d\right)^{\frac{h}{\delta}}}}{\left(\frac{p}{\delta}\right) !} \\
\leq & \frac{A_{1}(1+p)^{s_{0}} B_{1}^{h} d^{\frac{p-h}{\delta}} e^{-\frac{d(\cos \phi)}{2}|\lambda|^{\delta}}}{\left(\frac{p-h}{\delta}\right) !} \quad \text { (by Lemma 5.2). }
\end{aligned}
$$

Suppose $h<0$. In the first case of $p \leq h\left(=h_{-}\right)$, we have $\hat{r}_{p, h, s}(\lambda)=0$ by Lemma 5.3 and $\hat{r}_{p, h}^{1}(\lambda)=0$. Secondly if $h<p \leq h_{+}(=0)$, then $\hat{r}_{p, h, s}(\lambda)=$ $p^{s} \hat{r}_{p, h}^{0}(\lambda)$ and by Lemma $5.3\left|\hat{r}_{p, h, s}(\lambda)\right| \leq A B^{|h|}(1+|p|)^{s_{0}} d^{\frac{p}{\delta}} e^{-\frac{d\left(\cos \phi_{0}\right)}{2}|\lambda|^{\delta}}\left(\frac{-p}{\delta}\right) !$. In the third case of $p>0$, we have for $|\lambda| \geq 1$

$$
\begin{aligned}
\left|\hat{r}_{p, h, s}(\lambda)\right| & \leq \frac{A B^{|h|}(1+|p|)^{s_{0}} d^{\frac{p}{\delta}} e^{-\frac{d\left(\cos \phi_{0}\right)}{2}|\lambda|^{\delta}}}{|\lambda|^{|h|}\left(\frac{p}{\delta}\right) !}+\frac{A d^{\frac{p}{\delta}}(1+p)^{s_{0}-1} e^{-\frac{3 d \cos \phi_{0}}{4}|\lambda|^{\delta}}}{|\lambda|^{|h|}\left(\frac{p}{\delta}\right) !} \\
& \leq \frac{A_{1} B_{1}^{|h|}(1+|p|)^{s_{0}} d^{\frac{p}{\delta}} e^{-\frac{d\left(\cos \phi_{0}\right)}{2}|\lambda|^{\delta}}}{\left(\frac{p}{\delta}\right) !} .
\end{aligned}
$$

This completes the proof of Proposition 2.3.

\section{§6. Estimate}

The purposes of this section are to give Lemma 6.1 used in the proof of Lemma 2.4 and to show Theorem 4.1, that is, to estimate $k_{p, q}^{n}\left(z, w^{\prime}\right)$. The latter is the main one.

Lemma 6.1. Let $g(t)$ be a continuous function on $[0, T](T>0)$ and $\gamma$ be a positive constant. Suppose that there exist positive constants $A$ and $B$ such that for any $n \in \mathbb{N}$

$$
|g(t)| \leq A B^{n} t^{n}\left(\frac{n}{\gamma}\right) ! \text { on }[0, T] .
$$

Then $|g(t)| \leq C_{0} A(B t)^{-\gamma / 2} e^{-(B t)^{-\gamma}}$ holds for a constant $C_{0}$ that is independent of $A$ and $B$. 
Proof. First we assume $B=1$. Let $n \in \mathbb{N}$ with $\gamma / n \leq T^{\gamma}$. Suppose that $\gamma /(n+1) \leq t^{\gamma} \leq \gamma / n$. Then, by (6.1) and Stirling's formula $(n / \gamma) ! \sim$ $\left(\frac{n}{\gamma}\right)^{n / \gamma} \sqrt{2 \pi \frac{n}{\gamma}} e^{-n / \gamma}$ as $n \rightarrow \infty$, there is a constant $C_{0}$ such that

$$
|g(t)| \leq A\left(\frac{\gamma}{n}\right)^{\frac{n}{\gamma}}\left(\frac{n}{\gamma}\right) ! \leq C_{0} A \sqrt{\frac{n}{\gamma}} e^{-(n+1) / \gamma} \leq C_{0} A \frac{e^{-t^{-\gamma}}}{t^{\gamma / 2}} .
$$

This means $|g(t)| \leq C_{0} A t^{-\gamma / 2} e^{-t^{-\gamma}}$ for $t \in[\gamma /(n+1), \gamma / n]$ and for all $n \in \mathbb{N}$ with $\gamma / n \leq T^{\gamma}$. So the assertion holds for $B=1$. By considering $g(t / B)$, we have the estimate of $g(t)$ for $B>0$.

In order to show Theorem 4.1 we need majorant functions. For formal power series of $N$ variables $z, A(z)=\sum_{\alpha} A_{\alpha} z^{\alpha}$ and $B(z)=\sum_{\alpha} B_{\alpha} z^{\alpha}$, we define $A(z) \ll B(z)$ by $\left|A_{\alpha}\right| \leq B_{\alpha}$ for all $\alpha \in \mathbb{N}^{N}$. $A(z) \gg 0$ means $A_{\alpha} \geq 0$ for all $\alpha \in \mathbb{N}^{N}$. Let $\psi^{(k)}(t)(k \in \mathbb{Z})$ be a sequence of majorant functions in one variable $t$,

$$
\begin{cases}\psi^{(k)}(t)=k ! /(r-t)^{k+1} & \text { for } \quad k \geq 0 \\ \psi^{(k)}(t)=\int_{0}^{t} \psi^{(k+1)}(\tau) d \tau & \text { for } \quad k<0\end{cases}
$$

where $r$ is some positive constant. Then $\psi^{(k)}(t) \gg 0, \frac{d \psi^{(k)}}{d t}(t)=\psi^{(k+1)}(t)$ and if $0<r \leq 1$, it holds that $\psi^{(k)}(t) \ll \psi^{(k+1)}(t)$. By modifying $\psi^{(k)}(t)$, let us define another family of majorant functions $\Psi_{k}^{(s)}(t)(k \in \mathbb{Z}, s \in \mathbb{N})$

$$
\Psi_{k}^{(s)}(t)=\left(\frac{d}{d t}\right)^{s}\left\{\frac{R^{\prime}}{R^{\prime}-t} \psi^{(k)}(t)\right\} \quad \text { where } \quad 0<r<R^{\prime}<1 .
$$

We have $\left(R^{\prime}-t\right) \Psi_{k}^{(s)}(t) \gg 0$ and

Lemma 6.2. (1) The following inequalities hold:

$$
\left\{\begin{array}{l}
\Psi_{k+1}^{(s)}(t) \gg \Psi_{k}^{(s)}(t), \quad \Psi_{k}^{(s)}(t) \gg \Psi_{k}^{(s-1)}(t), \quad \Psi_{k-1}^{(s)}(t) \gg \Psi_{k}^{(s-1)}(t) \\
\frac{1}{R-R^{\prime}} \Psi_{k}^{(s)}(t) \gg(R-t)^{-1} \Psi_{k}^{(s)}(t) \quad \text { for } \quad R^{\prime}<R
\end{array}\right.
$$

(2) If $k \geq 0$, then

$$
\psi^{(s+k)}(t) \ll \Psi_{k}^{(s)}(t) \ll \frac{R^{\prime}}{R^{\prime}-r} \psi^{(s+k)}(t) .
$$


(3) If $k<0$ and $R^{\prime}>2 r$, then

$$
\psi^{(s+k)}(t) \ll \Psi_{k}^{(s)}(t) \ll \frac{2^{|k|} R^{\prime}}{R^{\prime}-2 r} \psi^{(s+k)}(t) .
$$

(4) Let $|t| \leq r / 2$. Then

$$
\begin{aligned}
& \left|\psi^{(k)}(t)\right| \leq \frac{2^{k+1} k !}{r^{k+1}} \quad \text { for } \quad k \geq 0 \\
& \left|\psi^{(k)}(t)\right| \leq \frac{2|t|^{|k|}}{r|k| !} \quad \text { for } \quad k \leq 0 .
\end{aligned}
$$

(5) Let $|t| \leq r / 2, R^{\prime}>2 r, s \geq 0$ and $k \geq 0$. Then there exist constants $C_{0}$ and $C_{1}$ such that

$$
\left|\Psi_{-k}^{(s)}(t)\right| \leq \frac{C_{0} C_{1}^{k+s} s !}{k !}
$$

The proofs are not difficult and we refer them to [3] or [8] (see also [1] and [10]). Now we proceed to estimate $k_{p, q}^{n}\left(z, w^{\prime}\right)$. We assume the condition $\left(\mathrm{C}_{i}\right)$ for fixed $i \in\left\{0,1, \ldots, p^{*}-1\right\}$. Hence we have $e(i)=-k_{i}+l_{i}$ and assume $b_{\alpha(i)}(z)=1$, where $\alpha(i)=\left(k_{i}-l_{i}, l_{i}, 0, \ldots, 0\right)$ as in Section 3. Suppose also that the coefficients of $P\left(z, \partial_{z}\right)$ are holomorphic in $\left\{z \in \mathbb{C}^{d+1} ;\left|z_{i}\right| \leq R, 0 \leq i \leq d\right\}$ and let $r_{i}(0 \leq i \leq 2)$ be constants with $0<r_{0}<r_{1}<r_{2}<R$ as in the preceding sections. We use $\Psi_{k}^{(s)}(t)$ defined by (6.3) and assume $0<r<r_{0}$ and $2 r<R^{\prime}<R \leq 1$ to apply Lemma 6.2 to the following estimates. We have

Theorem 6.3. Suppose that $\left|z_{i}\right| \leq r_{0}<r_{1} \leq\left|w_{i}\right| \leq r_{2}$ for $1 \leq i \leq d$. Then there exist constants $A, B, c$ and $\rho_{0}>1$ such that for $\rho \geq \rho_{0}$ if $0 \leq i \leq p^{*}-2$,

$$
\begin{aligned}
& k_{p, q}^{n}\left(z, w^{\prime}\right) \ll A^{n+1} B^{p+e(i)+n+q} \frac{\rho^{(p+e(i)+n) / \delta_{i}}}{\rho^{n+q}} e^{c(1+|p|+q) z_{1}} \\
& \times\left(\sum_{r=0}^{+\infty}(1+p+e(i)+2 n+q)^{r} \Psi_{-\left[\frac{n}{\delta_{i+1}}\right]-q-r}^{\left(\left[\frac{p+e(i)+n}{\delta_{i}}\right]+m\right)}(t)\right)
\end{aligned}
$$

and if $i=p^{*}-1$,

$$
\begin{aligned}
& k_{p, q}^{0}\left(z, w^{\prime}\right) \ll A B^{p+e\left(p^{*}-1\right)+q} \frac{\rho^{\left(p+e\left(p^{*}-1\right)\right) / \delta_{p^{*}-1}}}{\rho^{q}} e^{c(1+|p|+q) z_{1}} \\
& \times\left(\sum_{r=0}^{+\infty}\left(1+p+e\left(p^{*}-1\right)+q\right)^{r} \Psi_{-q-r}^{\left(\left[\frac{p+e\left(p^{*}-1\right)}{\delta_{p^{*}-1}}\right]+m\right)}(t)\right),
\end{aligned}
$$


where $m$ is the order of $P\left(z, \partial_{z}\right), t=z_{0}+\rho z_{1}+\sum_{i=2}^{d} z_{i}, p \geq-e(i)-n$ and $q \geq 0$.

For our purpose let us write again the equations that $k_{p, q}^{n}\left(z, w^{\prime}\right)$ satisfy

$$
\left\{\begin{array}{c}
\mathcal{P}\left(p+e(i), q ; z, \partial_{z^{\prime}}\right) k_{p, q}^{n}\left(z, w^{\prime}\right) \\
+I_{0}^{n}(p+e(i), q)+\sum_{h=1}^{e(i)-e(p-1)} I_{h}^{n}(p+e(i), q)=0, \\
\partial_{z_{1}}^{l} k_{p, q}^{n}\left(z_{0}, 0, z^{\prime \prime}, w^{\prime}\right)=0 \quad \text { for } \quad 0 \leq l \leq l_{i}-1,
\end{array}\right.
$$

where

$$
\begin{aligned}
& \mathcal{P}\left(p+e(i), q ; z, \partial_{z^{\prime}}\right)=\sum_{\left\{(\alpha, s) ; e_{\alpha}=e(i), 0 \leq s \leq j_{\alpha}\right\}} C_{j_{\alpha}, q+\alpha_{0}, s, 0} p^{s} b_{\alpha, 0}(z) \partial_{z^{\prime}}^{\alpha^{\prime}}, \\
& \left.I_{0}^{n}(p+e(i), q)=\frac{\sum^{\prime}}{\left\{\begin{array}{l}
\left(\alpha, l, s, s^{\prime}, p^{\prime}, q^{\prime}\right) \\
p^{\prime}+l+e_{\alpha}=p+e(i), q^{\prime}+s^{\prime}=q, \\
e_{\alpha}-e(i) \geq 0,0 \leq l \leq \alpha_{0}, \\
s+s^{\prime} \leq j_{\alpha}, l+e_{\alpha}-e(i)+s^{\prime}>0
\end{array}\right.}\right\} \\
& b_{\alpha, l}(z) C_{j_{\alpha}, q^{\prime}+\alpha_{0}-l, s, s^{\prime}} p^{s} \partial_{z^{\prime}}^{\alpha^{\prime}} \partial_{z_{0}}^{l} k_{p^{\prime}, q^{\prime}}^{n}\left(z, w^{\prime}\right)
\end{aligned}
$$

and

$$
\begin{aligned}
I_{h}^{n}(p+e(i), q)= & \sum_{\left\{\begin{array}{c}
\left(\alpha, l, s, s^{\prime}, p^{\prime}, q^{\prime}\right) \\
p^{\prime}+l+e_{\alpha}=p+e(i), q^{\prime}+s^{\prime}=q, \\
e_{\alpha}-e(i)=-h, 0 \leq l \leq \alpha_{0}, \\
s+s^{\prime} \leq j_{\alpha}
\end{array}\right\}} b_{\alpha, l}(z) C_{j_{\alpha}, q^{\prime}+\alpha_{0}-l, s, s^{\prime}} p^{s} \partial_{z^{\prime}}^{\alpha^{\prime}} \partial_{z_{0}}^{l} k_{p^{\prime}, q^{\prime}}^{n-h}\left(z, w^{\prime}\right) .
\end{aligned}
$$

Before proving Theorem 6.3 we give a lemma.

Lemma 6.4. Let $u(z)$ be a solution of

$$
\left\{\begin{array}{l}
\mathcal{P}\left(p+e(i), q ; z, \partial_{z^{\prime}}\right) u(z)=f(z) \\
\partial_{z_{1}}^{l} u\left(z_{0}, 0, z^{\prime \prime}\right)=0 \quad \text { for } 0 \leq l \leq l_{i}-1 .
\end{array}\right.
$$

Suppose further that $f(z) \ll e^{c(1+|p|+q) z_{1}} \rho^{l_{i}} \Psi_{-b}^{a+l_{i}}(t), a, b \in \mathbb{N}$. Then there exist $c_{0}, \rho_{0} \geq 1$ and $A_{1}$ which are independent of $a$ and $b$ such that $u(z) \ll$ $A_{1} e^{c(1+|p|+q) z_{1}} \Psi_{-b}^{a}(t)$ holds for $c \geq c_{0}$ and $\rho \geq \rho_{0}$.

Proof. We may assume $c \geq 1$. Put

$$
\mathcal{P}^{\prime}\left(p+e(i), q ; z, \partial_{z^{\prime}}\right)=\sum_{\left\{\begin{array}{c}
(\alpha, s), \alpha \neq \alpha(i) ; \\
e_{\alpha}=e(i), 0 \leq s \leq j_{\alpha}
\end{array}\right\}} C_{j_{\alpha}, q+\alpha_{0}, s, 0} p^{s} b_{\alpha, 0}(z) \partial_{z^{\prime}}^{\alpha^{\prime}}
$$


Then

$$
\partial_{z_{1}}^{l_{i}} u(z)=-\mathcal{P}^{\prime}\left(p+e(i), q ; z, \partial_{z^{\prime}}\right) u+f(z)
$$

Since $\left|C_{j_{\alpha}, q+\alpha_{0}, s, 0} p^{s}\right| \leq C_{0}(1+q)^{j_{\alpha}-s}|p|^{s} \leq C_{0}(1+|p|+q)^{j_{\alpha}}$ by Lemma 3.2,

$$
\begin{aligned}
& C_{j_{\alpha}, q+\alpha_{0}, s, 0} p^{s} b_{\alpha, 0}(z) \partial_{z^{\prime}}^{\alpha^{\prime}}\left(e^{c(1+|p|+q) z_{1}} \Psi_{-b}^{a}(t)\right) \\
& \ll C(1+|p|+q)^{j_{\alpha}} e^{c(1+|p|+q) z_{1}}\left(\sum_{l=0}^{\alpha_{1}}\left(\begin{array}{c}
\alpha_{1} \\
l
\end{array}\right)(c(1+|p|+q))^{\alpha_{1}-l} \rho^{l} \Psi_{-b}^{a+\left|\alpha^{\prime \prime}\right|+l}(t)\right) \\
& \ll C^{\prime} e^{c(1+|p|+q) z_{1}}\left(\sum_{l=0}^{\alpha_{1}} c^{\alpha_{1}-l}(1+|p|+q)^{j_{\alpha}+\alpha_{1}-l} \rho^{l} \Psi_{-b}^{a+\left|\alpha^{\prime \prime}\right|+l}(t)\right) .
\end{aligned}
$$

It follows from the relation $e_{\alpha}=e(i)$, that is, $j_{\alpha}-\alpha_{0}=-k_{i}+l_{i}$ that $j_{\alpha}+\alpha_{1}=$ $\alpha_{0}-k_{i}+l_{i}+\alpha_{1}=l_{i}-\left|\alpha^{\prime \prime}\right|-k_{i}+|\alpha| \leq l_{i}-\left|\alpha^{\prime \prime}\right|$. Hence it holds that

$$
\begin{aligned}
& C_{j_{\alpha}, q+\alpha_{0}, s, 0} p^{s} b_{\alpha, 0}(z) \partial_{z^{\prime}}^{\alpha^{\prime}}\left(e^{c(1+|p|+q) z_{1}} \Psi_{-b}^{a}(t)\right) \\
\ll & C^{\prime} e^{c(1+|p|+q) z_{1}}\left(\sum_{l=\left|\alpha^{\prime \prime}\right|}^{\left|\alpha^{\prime}\right|} c^{\left|\alpha^{\prime}\right|-l}(1+|p|+q)^{l_{i}-l} \rho^{l-\left|\alpha^{\prime \prime}\right|} \Psi_{-b}^{a+l}(t)\right)
\end{aligned}
$$

and

$$
\begin{aligned}
& -\mathcal{P}^{\prime}\left(p+e(i), q ; z, \partial_{z^{\prime}}\right)\left(A_{1} e^{c(1+|p|+q) z_{1}} \Psi_{-b}^{a}(t)\right)+f(z) \ll e^{c(1+|p|+q) z_{1}} \\
& \times \sum_{\substack{\alpha \neq \alpha(i) ; \\
e_{\alpha}=e(i), 0 \leq s \leq j_{\alpha}}} A_{1} C^{\prime}\left(\sum_{l=\left|\alpha^{\prime \prime}\right|}^{\left|\alpha^{\prime}\right|} c^{\left|\alpha^{\prime}\right|-l} \rho^{l-\left|\alpha^{\prime \prime}\right|}(1+|p|+q)^{l_{i}-l} \Psi_{-b}^{a+l}(t)\right) \\
& +e^{c(1+|p|+q) z_{1}} \rho^{l_{i}} \Psi_{-b}^{a+l_{i}}(t) .
\end{aligned}
$$

On the other hand

$$
\begin{aligned}
& \partial_{z_{1}}^{l_{i}}\left(A_{1} e^{c(1+|p|+q) z_{1}} \Psi_{-b}^{a}(t)\right) \\
= & A_{1} e^{c(1+|p|+q) z_{1}} \sum_{l=0}^{l_{i}}\left(\begin{array}{c}
l_{i} \\
l
\end{array}\right) c^{l_{i}-l} \rho^{l}(1+|p|+q)^{l_{i}-l} \Psi_{-b}^{a+l}(t) .
\end{aligned}
$$

For $\alpha \neq \alpha(i)$ with $e_{\alpha}=e(i)$ we have $l_{i} \geq\left|\alpha^{\prime}\right|$, moreover, $\left|\alpha^{\prime}\right|<l_{i}$ or $\left|\alpha^{\prime \prime}\right|>0$ holds. So $\lim _{\rho \rightarrow \infty, c \rightarrow \infty} \frac{c^{l_{i}-l} \rho^{l}}{c^{\alpha^{\prime} \mid-l} \rho^{l-\left|\alpha^{\prime \prime}\right|}}=\infty$ and there exist $c_{0}>1, \rho_{0}>1$ and 
$A_{1}$ such that if $c \geq c_{0}$ and $\rho \geq \rho_{0}$,

$$
\begin{aligned}
& \partial_{z_{1}}^{l_{i}} A_{1} e^{c(1+|p|+q) z_{1}} \Psi_{-b}^{a}(t) \\
& \gg-\mathcal{P}^{\prime}\left(p+e(i), q ; z, \partial_{z^{\prime}}\right) A_{1} e^{c(1+|p|+q) z_{1}} \Psi_{-b}^{a}(t)+f(z),
\end{aligned}
$$

from which $u(z) \ll A_{1} e^{c(1+|p|+q) z_{1}} \Psi_{-b}^{a}(t)$ follows.

Proof of Theorem 6.3. We give the proof of (6.9). We can show (6.10) in the similar way. So $0 \leq i \leq p^{*}-2$ and we show (6.9) by induction. Let $n=0$ and $(p, q)=(-e(i), 0)$. Then

$$
\begin{aligned}
& \mathcal{P}\left(0,0 ; z, \partial_{z^{\prime}}\right) k_{-e(i), 0}^{0}\left(z, w^{\prime}\right)=f(z), f(z)=\frac{1}{(2 \pi i)^{d} \prod_{j=1}^{d}\left(w_{j}-z_{j}\right)}, \\
& \partial_{z_{1}}^{l} k_{-e(i), 0}^{0}\left(z_{0}, 0, z^{\prime \prime}\right)=0 \quad \text { for } \quad 0 \leq l<l_{i} .
\end{aligned}
$$

Since $\left|z_{i}\right| \leq r_{0}<r_{1} \leq\left|w_{i}\right| \leq r_{2}$, we have $f(z) \ll M \rho^{l_{i}} \Psi_{0}^{m+l_{i}}(t)$ for some $M>0$. Then it follows from Lemma 6.4 that there exist $c, \rho_{0} \geq 1$ and $A_{1}$ such that $u(z) \ll A_{1} M e^{c|e(i)| z_{1}} \Psi_{0}^{m}(t)$ for $\rho \geq \rho_{0}$.

Now assume that the estimate (6.9) of $k_{p^{\prime}, q^{\prime}}^{n^{\prime}}\left(z, w^{\prime}\right)$ holds for $n^{\prime}<n$ and for $\left(n^{\prime}, p^{\prime}, q^{\prime}\right)$ with $n^{\prime}=n, p^{\prime}+q^{\prime}<p+q$. In the following we study the estimates under the inductive hypothesis. Let us return to (6.11). First we consider the estimate of $I_{h}^{n}(p+e(i), q)(h \geq 1)$ consisting of terms of $\partial_{z^{\prime}}^{\alpha^{\prime}} \partial_{z_{0}}^{l} k_{p^{\prime}, q^{\prime}}^{n-h}\left(z, w^{\prime}\right)$.

Lemma 6.5. There is a constant $A_{0}$ such that

$$
\begin{gathered}
\sum_{h=1}^{e(i)-e\left(p^{*}-1\right)} I_{h}^{n}(p+e(i), q) \ll A_{0} A^{n} B^{p+e(i)+n+q} \frac{\rho^{(p+e(i)+n) / \delta_{i}+l_{i}-1}}{\rho^{n+q}} \\
\times e^{c(1+|p|+q) z_{1}}\left(\sum_{r=0}^{+\infty}(1+p+e(i)+2 n+q)^{r} \Psi \begin{array}{c}
\left(\left[\frac{p+n}{\delta_{i}}\right]+m+l_{i}\right) \\
-\left[\frac{n}{\delta_{i+1}}\right]-q-r
\end{array}\right) .
\end{gathered}
$$

Proof. It follows from the inductive hypothesis that

$$
\begin{aligned}
& \partial_{z^{\prime}}^{\alpha^{\prime}} \partial_{z_{0}}^{l} k_{p^{\prime}, q^{\prime}}^{n-h}\left(z, w^{\prime}\right) \ll A^{n-h+1} B^{p^{\prime}+e(i)+n-h+q^{\prime}} \frac{\rho^{\left(p^{\prime}+e(i)+n-h\right) / \delta_{i}+\left|\alpha^{\prime}\right|}}{\rho^{n-h+q^{\prime}}} \\
& \times e^{c\left(1+\left|p^{\prime}\right|+q^{\prime}\right) z_{1}}\left(\sum_{r^{\prime}=0}^{+\infty}\left(1+p^{\prime}+e(i)+2(n-h)+q^{\prime}\right)^{r^{\prime}}\right. \\
& \left.\times\left(\sum_{r^{\prime \prime}=0}^{\alpha_{1}}\left(\begin{array}{l}
\alpha_{1} \\
r^{\prime \prime}
\end{array}\right)\left(c\left(1+\left|p^{\prime}\right|+q^{\prime}\right)\right)^{r^{\prime \prime}} \Psi_{-\left[\frac{n-h}{\delta_{i+1}}\right]-q^{\prime}-r^{\prime}}^{\left(\left[\frac{p^{\prime}+e(i)+n-h}{\delta_{i}}\right]+m+l+\left|\alpha^{\prime}\right|-r^{\prime \prime}\right)}(t)\right)\right) .
\end{aligned}
$$

By the relations $h=e(i)-e_{\alpha}, e(i)-e_{\alpha} \leq \gamma_{i+1}\left(k_{i}-|\alpha|\right), p^{\prime}+l+e_{\alpha}=p+e(i)$, $p^{\prime}+e(i)+n-h \geq 0$ and $q^{\prime}+s^{\prime}=q$, there is a constant $C$ such that $\left(1+\left|p^{\prime}\right|+q^{\prime}\right) \leq$ 
$C_{0}\left(1+p^{\prime}+e(i)+2(n-h)+q^{\prime}\right)$ for $p^{\prime} \geq-e(i)-(n-h)$ and $q^{\prime} \geq 0$. Hence by Lemma 6.2 and by replacing $r^{\prime}+r^{\prime \prime}$ by $r^{\prime}$, we have

$$
\begin{aligned}
& \partial_{z^{\prime}}^{\alpha^{\prime}} \partial_{z_{0}}^{l} k_{p^{\prime}, q^{\prime}}^{n-h}\left(z, w^{\prime}\right) \\
\ll & C_{1} A^{n-h+1} B^{p^{\prime}+e(i)+n-h+q^{\prime}} \frac{\rho^{\left(p^{\prime}+e(i)+n-h\right) / \delta_{i}+\left|\alpha^{\prime}\right|}}{\rho^{n-h+q^{\prime}}} e^{c\left(1+\left|p^{\prime}\right|+q^{\prime}\right) z_{1}} \\
\times & \left(\sum_{r^{\prime}=0}^{+\infty}\left(1+p^{\prime}+e(i)+2(n-h)+q^{\prime}\right)^{r^{\prime}} \Psi_{-\left[\frac{n-h}{\delta_{i+1}}\right]-q^{\prime}-r^{\prime}}^{\left(\left[\frac{p^{\prime}+e(i)+n-h}{\delta_{i}}\right]+m+l+\left|\alpha^{\prime}\right|\right)}(t)\right) .
\end{aligned}
$$

It holds by the properties of $\gamma_{i}$ and $\delta_{i}$ that

$$
\begin{aligned}
& -\left[\frac{n-h}{\delta_{i+1}}\right]-q^{\prime}-r^{\prime}=-\left[\frac{n}{\delta_{i+1}}-\frac{h}{\gamma_{i+1}}\right]-q^{\prime}-r^{\prime}+h \\
\leq & -\left[\frac{n}{\delta_{i+1}}\right]-q^{\prime}-r^{\prime}+h+k_{i}-|\alpha|=-\left[\frac{n}{\delta_{i+1}}\right]-q^{\prime}-r^{\prime}+e(i)-e_{\alpha}+k_{i}-|\alpha| \\
= & -\left[\frac{n}{\delta_{i+1}}\right]-q^{\prime}-r^{\prime}-j_{\alpha}+l_{i}-\left|\alpha^{\prime}\right|=-\left[\frac{n}{\delta_{i+1}}\right]-q-r^{\prime}+s^{\prime}-j_{\alpha}+l_{i}-\left|\alpha^{\prime}\right|, \\
& {\left[\frac{p^{\prime}+e(i)+n-h}{\delta_{i}}\right]+m+l+\left|\alpha^{\prime}\right| } \\
= & {\left[\frac{p-l-e_{\alpha}+2 e(i)+n-h}{\delta_{i}}\right]+m+l+\left|\alpha^{\prime}\right| \leq\left[\frac{p+e(i)+n}{\delta_{i}}\right]+m+\left|\alpha^{\prime}\right| . }
\end{aligned}
$$

By Lemma 6.2-(1) there is a constant $C_{1}$ such that

$$
\begin{aligned}
e^{c\left(1+\left|p^{\prime}\right|+q^{\prime}\right) z_{1}} \Psi_{k}^{(s)}(t) & \ll e^{c(1+|p|+q) z_{1}} e^{c\left|e(i)-e_{\alpha}-l\right| z_{1}} \Psi_{k}^{(s)}(t) \\
& \ll C_{1} e^{c(1+|p|+q) z_{1}} \Psi_{k}^{(s)}(t) .
\end{aligned}
$$

Thus by the bound of $C_{j_{\alpha}, q^{\prime}+\alpha_{0}-l, s, s^{\prime}}$ (see Lemma 3.2) and Lemma 6.2 we have

$$
\begin{aligned}
& b_{\alpha, l}(z) C_{j_{\alpha}, q^{\prime}+\alpha_{0}-l, s, s^{\prime}} p^{\prime s} \partial_{z^{\prime}}^{\alpha^{\prime}} \partial_{z_{0}}^{l} k_{p^{\prime}, q^{\prime}}^{n-h}\left(z, w^{\prime}\right) \ll A_{1} A^{n-h+1} B^{p^{\prime}+e(i)+n-h+q^{\prime}} \\
& \times\left(1+q^{\prime}\right)^{j_{\alpha}-s-s^{\prime}}\left|p^{\prime}\right|^{s} \frac{\rho^{\left(p^{\prime}+e(i)+n-h\right) / \delta_{i}+\left|\alpha^{\prime}\right|}}{\rho^{n-h+q^{\prime}}} e^{c(1+|p|+q) z_{1}} \\
& \times\left(\sum_{r^{\prime}=0}^{+\infty}\left(1+p+e(i)+2 n+q^{\prime}\right)^{r^{\prime}} \Psi_{-\left[\frac{n}{\delta_{i+1}}\right]-q-r^{\prime}+s^{\prime}-j_{\alpha}+l_{i}-\left|\alpha^{\prime}\right|}^{\left(\left[\frac{p++e(i)+n}{\delta_{i}}\right]+m+\left|\alpha^{\prime}\right|\right)}(t)\right) \\
& \ll A_{1} A^{n-h+1} B^{p-l+e(i)+n+q^{\prime}}\left(1+\left|p^{\prime}\right|+q^{\prime}\right)^{j_{\alpha}-s^{\prime}} \frac{\rho^{(p-l+e(i)+n) / \delta_{i}+\left|\alpha^{\prime}\right|}}{\rho^{n-h+q^{\prime}}} \\
& \times e^{c(1+|p|+q) z_{1}}\left(\sum_{r^{\prime}=0}^{+\infty}\left(1+p+e(i)+2 n+q^{\prime}\right)^{r^{\prime}} \Psi_{-\left[\frac{n}{\delta_{i+1}}\right]-q-r^{\prime}+s^{\prime}-j_{\alpha}+l_{i}-\left|\alpha^{\prime}\right|}^{\left(\left[\frac{p+e(i)+n}{\delta_{i}}\right]+m+\left|\alpha^{\prime}\right|\right)}(t)\right) .
\end{aligned}
$$


Note inequalities $\left(1+|p|+q^{\prime}\right) \leq C\left(1+p+e(i)+2 n+q^{\prime}\right)$ for $p \geq-e(i)-n, q^{\prime} \geq 0$, and $h+s^{\prime}+\left|\alpha^{\prime}\right|=e(i)-e_{\alpha}+s^{\prime}+\left|\alpha^{\prime}\right| \leq e(i)-e_{\alpha}+j_{\alpha}+\left|\alpha^{\prime}\right| \leq|\alpha|-k_{i}+l_{i} \leq l_{i}-1$. Therefore we have $\rho^{\left|\alpha^{\prime}\right|-\left(n-h+q^{\prime}\right)}=\rho^{\left(h+s^{\prime}+\left|\alpha^{\prime}\right|\right)-(n+q)} \leq \rho^{\left(l_{i}-1\right)-(n+q)}$ for $\rho \geq 1$ and

$$
\begin{aligned}
& b_{\alpha, l}(z) C_{j_{\alpha}, q^{\prime}+\alpha_{0}-l, s, s^{\prime}} p^{s} \partial_{z^{\prime}}^{\alpha^{\prime}} \partial_{z_{0}}^{l} k_{p^{\prime}, q^{\prime}}^{n-h}\left(z, w^{\prime}\right) \\
& \ll A_{1} A^{n-h+1} B^{p-l+e(i)+n+q^{\prime}} \frac{\rho^{(p-l+e(i)+n) / \delta_{i}+l_{i}-1}}{\rho^{n+q}} \\
& \times e^{c(1+|p|+q) z_{1}}\left(\sum_{r^{\prime}=0}^{+\infty}\left(1+p+e(i)+2 n+q^{\prime}\right)^{r^{\prime}+j_{\alpha}-s^{\prime}}\right. \\
& \left.\times \underset{\quad}{\left(\left[\frac{p+e(i)+n}{\delta_{i}}\right]+m+\left|\alpha^{\prime}\right|\right)}(t)\right) \ll A_{2} A^{n-h+1} B^{p+e(i)+n+q} \frac{\rho^{(p+e(i)+n) / \delta_{i}+l_{i}-1}}{\rho^{n+q}} \\
& \times e^{c(1+|p|+q) z_{1}}\left(\sum_{r=0}^{+\infty}(1+p+e(i)+2 n+q)^{r} \Psi_{-\left[\frac{n}{\delta_{i+1}}\right]-q-r+l_{i}-\left|\alpha^{\prime}\right|}^{\left(\left[\frac{p+e(i)+n}{\delta_{i}}\right]+m+\left|\alpha^{\prime}\right|\right)}(t)\right) \\
& \ll A_{2} A^{n-h+1} B^{p+e(i)+n+q} \frac{\rho^{(p+e(i)+n) / \delta_{i}+l_{i}-1}}{\rho^{n+q}} \\
& \times e^{c(1+|p|+q) z_{1}}\left(\sum_{r=0}^{+\infty}(1+p+e(i)+2 n+q)^{r} \Psi_{-\left[\frac{n}{\delta_{i+1}}\right]-q-r}^{\left(\left[\frac{p+e(i)+n}{\delta_{i}}\right]+m+l_{i}\right)}(t)\right) .
\end{aligned}
$$

$I_{h}^{n}(p+e(i), q)\left(1 \leq h \leq e(i)-e\left(p^{*}-1\right)\right)$ is a finite sum of terms estimated as above. Consequently the estimate (6.15) holds.

Next we estimate $I_{0}^{n}(p+e(i), q)$.

Lemma 6.6. There is a constant $A_{0}$ such that

$$
\begin{aligned}
& I_{0}^{n}(p+e(i), q) \ll A_{0} A^{n+1} B^{p+e(i)+n+q-1} \frac{\rho^{(p+e(i)+n) / \delta_{i}+l_{i}}}{\rho^{n+q}} \\
& \quad \times e^{c(1+|p|+q) z_{1}}\left(\sum_{r=0}^{+\infty}(1+p+e(i)+2 n+q)^{r} \Psi_{-\left[\frac{n}{\delta_{i+1}}\right]-q-r}^{\left(\left[\frac{p+e(i)+n}{\delta_{i}}\right]+m+l_{i}\right)}(t)\right) .
\end{aligned}
$$

Proof. Notice that $I_{0}^{n}(p+e(i), q)$ is determined by $k_{p^{\prime}, q^{\prime}}^{n}\left(z, w^{\prime}\right)$ with $p^{\prime}+$ $q^{\prime}<p+q$. From the inductive hypothesis we have in the same way as (6.16) 


$$
\begin{gathered}
\partial_{z^{\prime}}^{\alpha^{\prime}} \partial_{z_{0}}^{l} k_{p^{\prime}, q^{\prime}}^{n}\left(z, w^{\prime}\right) \ll C_{1} A^{n+1} B^{p^{\prime}+e(i)+n+q^{\prime}} \frac{\rho^{\left(p^{\prime}+e(i)+n\right) / \delta_{i}+\left|\alpha^{\prime}\right|}}{\rho^{n+q^{\prime}}} \\
\times e^{c\left(1+\left|p^{\prime}\right|+q^{\prime}\right) z_{1}}\left(\sum_{r^{\prime}=0}^{+\infty}\left(1+p^{\prime}+e(i)+2 n+q^{\prime}\right)^{r^{\prime}} \Psi_{-\left[\frac{n}{\delta_{i+1}}\right]-q^{\prime}-r^{\prime}}^{\left(\left[\frac{p^{\prime}+e(i)+n}{\delta_{i}}\right]+m+l+\left|\alpha^{\prime}\right|\right)}(t)\right) .
\end{gathered}
$$

It follows from the properties of $\gamma_{i}$ and $\delta_{i}$ that

$$
\begin{aligned}
& {\left[\frac{p^{\prime}+e(i)+n}{\delta_{i}}\right]+m+l+\left|\alpha^{\prime}\right|=\left[\frac{p-l-e_{\alpha}+2 e(i)+n}{\delta_{i}}\right]+m+l+\left|\alpha^{\prime}\right| } \\
\leq & {\left[\frac{p+n-e_{\alpha}+2 e(i)}{\delta_{i}}\right]+m+\left|\alpha^{\prime}\right|=\left[\frac{p+e(i)+n}{\delta_{i}}-\frac{e_{\alpha}-e(i)}{\gamma_{i}}\right] } \\
& \quad-e_{\alpha}+e(i)+m+\left|\alpha^{\prime}\right| \leq\left[\frac{p+e(i)+n}{\delta_{i}}\right]-e_{\alpha}+e(i)-|\alpha|+k_{i}+m+\left|\alpha^{\prime}\right| \\
= & {\left[\frac{p+e(i)+n}{\delta_{i}}\right]-j_{\alpha}+l_{i}+m }
\end{aligned}
$$

holds. By this inequality and Lemma 6.2

$$
\begin{aligned}
& \Psi_{-\left[\frac{n}{\delta_{i+1}}\right]-q^{\prime}-r^{\prime}}^{\left[\frac{p^{\prime}+e(i)+n}{\delta_{i}}\right]+m+l+\left|\alpha^{\prime}\right|}(t) \ll \Psi_{-\left[\frac{n}{\delta_{i+1}}\right]-q^{\prime}-r^{\prime}}^{\left[\frac{p+e(i)+n}{\delta_{i}}\right]-j_{\alpha}+l_{i}+m}(t) \ll \Psi_{-\left[\frac{n}{\delta_{i+1}}\right]-q^{\prime}-r^{\prime}-j_{\alpha}}^{\left[\frac{p+e(i)+n}{\delta_{i}}\right]+l_{i}+m}(t) \\
& =\Psi^{\left[\frac{p+e(i)+n}{\delta_{i}}\right]+l_{i}+m}(t)
\end{aligned}
$$

holds. We also have

$$
\begin{aligned}
& \frac{p^{\prime}+e(i)+n}{\delta_{i}}+\left|\alpha^{\prime}\right|-q^{\prime}=\frac{p-l-e_{\alpha}+2 e(i)+n}{\delta_{i}}+\left|\alpha^{\prime}\right|-q+s^{\prime} \\
\leq & \frac{p+n-e_{\alpha}+2 e(i)}{\delta_{i}}+\left|\alpha^{\prime}\right|-q+s^{\prime}=\frac{p+e(i)+n}{\delta_{i}}-\frac{e_{\alpha}-e(i)}{\gamma_{i}}-e_{\alpha}+e(i) \\
& +\left|\alpha^{\prime}\right|-q+s^{\prime} \leq \frac{p+e(i)+n}{\delta_{i}}-e_{\alpha}+e(i)-|\alpha|+k_{i}+\left|\alpha^{\prime}\right|-q+s^{\prime} \\
= & \frac{p+e(i)+n}{\delta_{i}}-q+l_{i}-j_{\alpha}+s^{\prime} \leq \frac{p+e(i)+n}{\delta_{i}}-q+l_{i} .
\end{aligned}
$$


Therefore $\frac{\rho^{\left(p^{\prime}+e(i)+n\right) / \delta_{i}+\left|\alpha^{\prime}\right|}}{\rho^{n+q^{\prime}}} \leq \frac{\rho^{(p+e(i)+n) / \delta_{i}+l_{i}}}{\rho^{n+q}}$ for $\rho \geq 1$. By these inequalities and $l+e_{\alpha}-e(i)+s^{\prime}>0$ the following estimate holds:

$$
\begin{aligned}
& b_{\alpha, l}(z) C_{j_{\alpha}, q^{\prime}+\alpha_{0}-l, s, s^{\prime}} p^{s} \partial_{z^{\prime}}^{\alpha^{\prime}} \partial_{z_{0}}^{l} k_{p^{\prime}, q^{\prime}}^{n}\left(z, w^{\prime}\right) \\
& \ll A_{1} A^{n+1} B^{p^{\prime}+e(i)+n+q^{\prime}}\left(1+q^{\prime}\right)^{j_{\alpha}-s-s^{\prime}}\left|p^{\prime}\right|^{s} \frac{\rho^{\left(p^{\prime}+e(i)+n\right) / \delta_{i}+\left|\alpha^{\prime}\right|}}{\rho^{n+q^{\prime}}} \\
& \times e^{c(1+|p|+q) z_{1}}\left(\sum_{r^{\prime}=0}^{+\infty}\left(1+p^{\prime}+e(i)+2 n+q^{\prime}\right)^{r^{\prime}} \Psi_{-\left[\frac{n}{\delta_{i+1}}\right]-q-r^{\prime}+s^{\prime}-j_{\alpha}}^{\left[\frac{p+e(i)+n}{\delta_{i}}\right]+l_{i}+m}(t)\right) \\
& \ll A_{1} A^{n+1} B^{p+q^{\prime}-l+2 e(i)-e_{\alpha}+n}\left(1+q^{\prime}\right)^{j_{\alpha}-s-s^{\prime}}\left|p^{\prime}\right|^{s} \frac{\rho^{(p+e(i)+n) / \delta_{i}+l_{i}}}{\rho^{n+q}}
\end{aligned}
$$

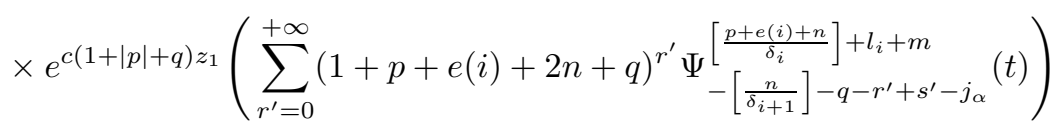

$$
\begin{aligned}
& \ll A_{1} A^{n+1} B^{p+e(i)+n+q-l+e(i)-e_{\alpha}-s^{\prime}} \frac{\rho^{(p+e(i)+n) / \delta_{i}+l_{i}}}{\rho^{n+q}} \\
& \times e^{c(1+|p|+q) z_{1}}\left(\sum_{r^{\prime}=0}^{+\infty}(1+p+e(i)+2 n+q)^{r^{\prime}+j_{\alpha}-s^{\prime}} \Psi_{-\left[\frac{n}{\delta_{i+1}}\right]-q-r^{\prime}+s^{\prime}-j_{\alpha}}^{\left[\frac{p+e(i)+n}{\delta_{i}}\right]+l_{i}+m}(t)\right) \\
& \ll A_{1}^{\prime} A^{n+1} B^{p+e(i)+n+q-1} \frac{\rho^{(p+e(i)+n) / \delta_{i}+l_{i}}}{\rho^{n+q}} \\
& \times e^{c(1+|p|+q) z_{1}}\left(\sum_{r=0}^{+\infty}\left(1+p+e(i)+2 n+q^{\prime}\right)^{r} \Psi_{-\left[\frac{n}{\delta_{i+1}}\right]-q-r}^{\left[\frac{p+e(i)+n}{\delta_{i}}\right]+l_{i}+m}(t)\right) .
\end{aligned}
$$

Consequently we have (6.17).

Now let us complete the proof of Theorem 6.3. We have assumed $0 \leq$ $i \leq p^{*}-2$. Return to (6.11). We have the estimates of $I_{h}^{n}(p+e(i), q)$ for $0 \leq h \leq e(i)-e\left(p^{*}-1\right)$. So it follows from Lemma 6.4 that there are constants $A$ and $B$ such that (6.9) holds for $k_{p, q}^{n}\left(z, w^{\prime}\right)$. As remarked at the beginning of the proof of Theorem 6.3, we can show (6.10) for $i=p^{*}-1$ in the same way as above.

Proof of Theorem 4.1. Let $0 \leq i \leq p^{*}-2$. Then it follows from (6.9) that there exists a constant $C$ such that 


$$
\begin{aligned}
& \partial_{z}^{\alpha} k_{p, q}^{n}\left(z, w^{\prime}\right) \ll A^{n+1} B^{p+e(i)+n+q} \frac{\rho^{(p+e(i)+n) / \delta_{i}}}{\rho^{n+q}} e^{c(1+|p|+q) z_{1}}\left(\sum_{r=0}^{+\infty} \sum_{r^{\prime}=0}^{\alpha_{1}}\right. \\
& \left.(1+p+e(i)+2 n+q)^{r}\left(\begin{array}{c}
\alpha_{1} \\
r^{\prime}
\end{array}\right) \rho^{\alpha_{1}-r^{\prime}}(c(1+|p|+q))^{r^{\prime}} \Psi_{-\left[\frac{n}{\delta_{i+1}}\right]-q-r}^{\left[\frac{p+e(i)+n}{\delta_{i}}\right]+m+|\alpha|-r^{\prime}}(t)\right) \\
& \ll A^{n+1} B^{p+e(i)+n+q}(C \rho)^{|\alpha|} \frac{\rho^{(p+e(i)+n) / \delta_{i}}}{\rho^{n+q}} e^{c(1+|p|+q) z_{1}} \\
& \times\left(\sum_{r=0}^{+\infty}(1+p+e(i)+2 n+q)^{r} \Psi_{-\left[\frac{n}{\delta_{i+1}}\right]-q-r}^{\left[\frac{p+e(i)+n}{\delta_{i}}\right]+m+|\alpha|}(t)\right) .
\end{aligned}
$$

Therefore if $|t| \leq r / 2$, by Lemma 6.2 there are constants $A_{1}, B_{1}, C_{1}$ and $D_{1}$ such that

$$
\begin{aligned}
\left|\partial_{z}^{\alpha} k_{p, q}^{n}\left(z, w^{\prime}\right)\right| & \leq A_{1}^{n+1} B_{1}^{p+e(i)+n+q} C_{1}^{|\alpha|} \frac{\rho^{(p+e(i)+n) / \delta_{i}+|\alpha|}}{\rho^{n+q}} \\
& \times\left(\frac{p+e(i)+n}{\delta_{i}}+m+|\alpha|\right) !\left(\sum_{r=0}^{+\infty} \frac{(1+p+e(i)+2 n+q)^{r} D_{1}^{r}}{\left(\left[\frac{n}{\delta_{i+1}}\right]+q+r\right) !}\right) .
\end{aligned}
$$

By the inequality

$$
\begin{aligned}
& \sum_{r=0}^{+\infty} \frac{(1+p+e(i)+2 n+q)^{r} D_{1}^{r}}{\left(\frac{n}{\delta_{i+1}}+q+r\right) !} \\
\leq & \frac{1}{\left(\frac{n}{\delta_{i+1}}\right) ! q !} \sum_{r=0}^{+\infty} \frac{(1+p+e(i)+2 n+q)^{r} D_{1}^{r}}{r !} \leq \frac{e^{(1+p+e(i)+2 n+q) D_{1}}}{\left(\frac{n}{\delta_{i+1}}\right) ! q !}
\end{aligned}
$$

we have (4.2a). Similarly we have (4.2b) from (6.10).

\section{References}

[1] Hamada, Y., Leray, J. et Wagschal, C., Système d'équation aux derivées partielles à caractéristic multiples; problème de Cauchy ramifié; hyperbolicité partielle, J. Math. Pures Appl., 55 (1976), 297-352.

[2] Kashiwara, M. et Schpira, P., Problème de Cauchy pour les systemes microdifferentiels dans le domain complexe, Inv. Math., 46 (1978), 17-38. 
[3] Komatsu, H., Irregularity of characteristic elements and construction of null solutions, J. Fac. Sci. Univ. Tokyo, 23 (1976), 297-342.

[4] Ouchi, S., Characteristic Cauchy problems and solutions of formal power series, Ann. Inst. Fourier, 33 (1983), 131-176.

[5] _ Existence of singular solutions and null solutions for linear partial differential operators, J. Fac. Sci. Univ. Tokyo, 32 (1985), 457-498.

[6] Singular solutions with asymptotic expansion of linear partial differential equations in the complex domain, Publ. RIMS, Kyoto Univ., 34 (1998), 291-311.

[7] Growth property and slowly increasing behavior of singular solutions of linear partial differential equations in the complex domain, J. Math. Soc. Japan, 52 (2000), 767-792.

[8] Asymptotic expansion of singular solutions and the characteristic polygon of linear partial differential equations in the complex domain, Publ. RIMS, Kyoto Univ., 36 (2000), 457-482.

[9] Persson, J., Singular holomorphic solutions of linear partial differential equations with holomorphic coefficients and nonanalytic solutions with analytic coefficients, Analytic solutions of partial differential equations (Trento 1981), Asterisque, 89-90 (1981), 223247.

[10] Wagschal, C., Problème de Cauchy analytique à données méromorphes, J. Math. Pures Appl., 51 (1972), 375-397. 